\title{
Functional and Banach Space Stochastic Calculi: Path-Dependent Kolmogorov Equations Associated with the Frame of a Brownian Motion
}

\author{
Andrea Cosso and Francesco Russo
}

\begin{abstract}
First, we revisit basic theory of functional Itô/path-dependent calculus, using the formulation of calculus via regularization. Relations with the corresponding Banach space valued calculus are explored. The second part of the paper is devoted to the study of the Kolmogorov type equation associated with the so called window Brownian motion, called path-dependent heat equation, for which well-posedness at the level of strict solutions is established. Then, a notion of strong approximating solution, called strong-viscosity solution, is introduced which is supposed to be a substitution tool to the viscosity solution. For that kind of solution, we also prove existence and uniqueness.
\end{abstract}

Keywords Horizontal and vertical derivative - functional Itô/path-dependent calculus $\cdot$ Banach space stochastic calculus $\cdot$ Strong-viscosity solutions $\cdot$ Calculus via regularization

2010 Math Subject Classification: $35 \mathrm{D} 35 \cdot 35 \mathrm{D} 40 \cdot 35 \mathrm{~K} 10 \cdot 60 \mathrm{H} 05 \cdot 60 \mathrm{H} 10$. $60 \mathrm{H} 30$

\section{Introduction}

The present work collects several results obtained in the papers $[9,10]$, focusing on the study of some specific examples and particular cases, for which an ad hoc analysis is developed. This work is an improved version of [8], trying to explain more

\footnotetext{
A. Cosso

Laboratoire de Probabilités et Modèles Aléatoires,

CNRS, UMR 7599, Université Paris Diderot, Paris, France

e-mail: andrea.cosso@math.univ-paris-diderot.fr

F. Russo $(\varangle)$

Unité de Mathématiques Appliquées, ENSTA ParisTech,

Université Paris-Saclay, 828, Boulevard des Maréchaux, 91120 Palaiseau, France

e-mail: francesco.russo@ensta-paristech.fr 
precisely some details. For example, in [8] a slightly more restrictive definition of strong-viscosity solution was adopted, see Remark 12.

Recently, a new branch of stochastic calculus has appeared, known as functional Itô calculus, which results to be an extension of classical Itô calculus to functionals depending on the entire path of a stochastic process and not only on its current value, see Dupire [17], Cont and Fournié [5-7]. Independently, Di Girolami and Russo, and more recently Fabbri, Di Girolami, and Russo, have introduced a stochastic calculus via regularizations for processes taking values in a separable Banach space $B$ (see [12-16]), including the case $B=C([-T, 0])$, which concerns the applications to the path-dependent calculus.

In the first part of the present paper, we follow [9] and revisit functional Itô calculus by means of stochastic calculus via regularization. We recall that Cont and Fournié [5-7] developed functional Itô calculus and derived a functional Itô's formula using discretization techniques of Föllmer [23] type, instead of regularization techniques, which in our opinion, better fit to the notion of derivative. Let us illustrate another difference with respect to [5]. One of the main issues of functional Itô calculus is the definition of the functional (or pathwise) derivatives, i.e., the horizontal derivative (calling in only the past values of the trajectory) and the vertical derivative (calling in only the present value of the trajectory). In [5], it is essential to consider functionals defined on the space of càdlàg trajectories, since the definition of functional derivatives necessitates of discontinuous paths. Therefore, if a functional is defined only on the space of continuous trajectories (because, e.g., it depends on the paths of a continuous process as Brownian motion), we have to extend it anyway to the space of càdlàg trajectories, even though, in general, there is no unique way to extend it. In contrast to this approach, we introduce a new space larger than the space of continuous trajectories $C([-T, 0])$, denoted by $\mathscr{C}([-T, 0])$, which allows us to define functional derivatives. $\mathscr{C}([-T, 0])$ is the space of bounded trajectories on $[-T, 0]$, continuous on $[-T, 0[$ and with possibly a jump at 0 . We endow $\mathscr{C}([-T, 0])$ with a topology such that $C([-T, 0])$ is dense in $\mathscr{C}([-T, 0])$ with respect to this topology. Therefore, any functional $\mathscr{U}:[0, T] \times C([-T, 0]) \rightarrow \mathbb{R}$, continuous with respect to the topology of $\mathscr{C}([-T, 0])$, admits a unique extension to $\mathscr{C}([-T, 0])$, denoted $u:[0, T] \times \mathscr{C}([-T, 0]) \rightarrow \mathbb{R}$. We present some significant functionals for which a continuous extension exists. Then, we develop the functional Itô calculus for $u:[0, T] \times \mathscr{C}([-T, 0]) \rightarrow \mathbb{R}$.

Notice that we use a slightly different notation compared with [5]. In particular, in place of a map $\mathscr{U}:[0, T] \times C([-T, 0]) \rightarrow \mathbb{R}$, in [5] a family of maps $F=\left(F_{t}\right)_{t \in[0, T]}$, with $F_{t}: C([0, t]) \rightarrow \mathbb{R}$, is considered. However, we can always move from one formulation to the other. Indeed, given $F=\left(F_{t}\right)_{t \in[0, T]}$, where each $F_{t}: C([0, t]) \rightarrow \mathbb{R}$, we can define $\mathscr{U}:[0, T] \times C([-T, 0]) \rightarrow \mathbb{R}$ as follows:

$$
\mathscr{U}(t, \eta):=F_{t}\left(\left.\eta(\cdot+T)\right|_{[0, t]}\right), \quad(t, \eta) \in[0, T] \times C([-T, 0]) .
$$

Vice-versa, let $\mathscr{U}:[0, T] \times C([-T, 0]) \rightarrow \mathbb{R}$ and define $F=\left(F_{t}\right)_{t \in[0, T]}$ as

$$
F_{t}(\tilde{\eta}):=\mathscr{U}(t, \eta), \quad(t, \tilde{\eta}) \in[0, T] \times C([0, t]),
$$


where $\eta$ is the element of $C([-T, 0])$ obtained from $\tilde{\eta}$ firstly translating $\tilde{\eta}$ on the interval $[-t, 0]$, then extending it in a constant way up to $-T$, namely $\eta(x):=$ $\tilde{\eta}(x+t) 1_{[-t, 0]}(x)+\tilde{\eta}(-t) 1_{[-T,-t)}(x)$, for any $x \in[-T, 0]$. Observe that, in principle, the map $\mathscr{U}$ contains more information than $F$, since in (1) we do not take into account the values of $\mathscr{U}$ at $(t, \eta) \in[0, T] \times C([-T, 0])$ with $\eta$ not constant on the interval $[-T,-t]$. Despite this, the equivalence between the two notations is guaranteed; indeed, when we consider the composition of $\mathscr{U}$ with a stochastic process, this extra information plays no role. Our formulation has two advantages. Firstly, we can work with a single map instead of a family of maps. In addition, the time variable and the path have two distinct roles in our setting, as for the time variable and the space variable in the classical Itô calculus. This, in particular, allows us to define the horizontal derivative independently of the time derivative, so that, the horizontal derivative defined in [5] corresponds to the sum of our horizontal derivative and of the time derivative. We mention that an alternative approach to functional derivatives was introduced in [1].

We end the first part of the paper showing how our functional Itô's formula is strictly related to the Itô's formula derived in the framework of Banach space valued stochastic calculus via regularization, for the case of window processes. This new branch of stochastic calculus has been recently conceived and developed in many directions in [12, 14-16]; for more details see [13]. For the particular case of window processes, we also refer to Theorem 6.3 and Sect. 7.2 in [12]. In the present paper, we prove formulae which allow to express functional derivatives in terms of differential operators arising in the Banach space valued stochastic calculus via regularization, with the aim of identifying the building blocks of our functional Itô's formula with the terms appearing in the Itô's formula for window processes.

Dupire [17] introduced also the concept of path-dependent partial differential equation, to which the second part of the present paper is devoted. Di Girolami and Russo, in Chap. 9 of [13], considered existence of regular solutions associated with a path dependent heat equation (which is indeed the Kolmogorov equation related to window Brownian motion) with a Fréchet smooth final condition. This was performed in the framework of Banach space valued calculus, for which we refer also to [22]. A flavour of the notion of regular solution in the Banach space framework, appeared in Chap. IV of [30] which introduced the notion of weak infinitesimal generator (in some weak sense) of the window Brownian motion and more general solutions of functional dependent stochastic differential equations. Indeed, the monograph [30] by Mohammed constitutes an excellent early contribution to the theory of this kind of equations.

We focus on semilinear parabolic path-dependent equations associated to the window Brownian motion. For more general equations we refer to [9] (for strict solutions) and to [10] (for strong-viscosity solutions). First, we consider regular solution, which we call strict solutions, in the framework of functional Itô calculus. We prove a uniqueness result for this kind of solution, showing that, if a strict solution exists, then it can be expressed through the unique solution to a certain backward stochastic differential equation (BSDE). Then, we prove an existence result for strict solutions. 
However, this notion of solution turns out to be unsuitable to deal with all significant examples. As a matter of fact, if we consider the path-dependent PDE arising in the hedging problem of lookback contingent claims, we can not expect too much regularity of the solution (this example is studied in detail in Sect.3.2). Therefore, we are led to consider a weaker notion of solution. In particular, we are interested in a viscosity-type solution, namely a solution which is not required to be differentiable.

The issue of providing a suitable definition of viscosity solutions for pathdependent PDEs has attracted a great interest, see Peng [33] and Tang and Zhang [42], Ekren et al. [18-20], Ren et al. [34]. In particular, the definition of viscosity solution provided by $[18-20,34]$ is characterized by the fact that the classical minimum/maximum property, which appears in the standard definition of viscosity solution, is replaced with an optimal stopping problem under nonlinear expectation [21]. Then, probability plays an essential role in this latter definition, which can not be considered as a purely analytic object as the classical definition of viscosity solution is; it is, more properly, a probabilistic version of the classical definition of viscosity solution. We also emphasize that a similar notion of solution, called stochastic weak solution, has been introduced in the recent paper [29] in the context of variational inequalities for the Snell envelope associated to a non-Markovian continuous process $X$. Those authors also revisit functional Itô calculus, making use of stopping times. This approach seems very promising. Instead, our aim is to provide a definition of viscosity type solution, which has the peculiarity to be a purely analytic object; this will be called a strong-viscosity solution to distinguish it from the classical notion of viscosity solution. A strong-viscosity solution to a path-dependent partial differential equation is defined, in a few words, as the pointwise limit of strict solutions to perturbed equations. We notice that the definition of strong-viscosity solution is similar in spirit to the vanishing viscosity method, which represents one of the primitive ideas leading to the conception of the modern definition of viscosity solution. Moreover, it has also some similarities with the definition of good solution, which turned out to be equivalent to the definition of $L^{p}$-viscosity solution for certain fully nonlinear partial differential equations, see, e.g., [3, 11, 27, 28]. Finally, our definition is likewise inspired by the notion of strong solution (which justifies the first word in the name of our solution), as defined for example in [2, 24, 25], even though strong solutions are required to be more regular (this regularity is usually required to prove uniqueness of strong solutions, which for example in [24, 25] is based on a Fukushima-Dirichlet decomposition). Instead, our definition of strong-viscosity solution to the path-dependent semilinear Kolmogorov equation is not required to be continuous, as in the spirit of viscosity solutions. The term viscosity in the name of our solution is also justified by the fact that in the finite dimensional case we have an equivalence result between the notion of strong-viscosity solution and that of viscosity solution, see Theorem 3.7 in [8]. We prove a uniqueness theorem for strong-viscosity solutions using the theory of backward stochastic differential equations and we provide an existence result. We refer to [10] for more general results (when the path-dependent equation is not the path-dependent heat equation) and also for the application of strong-viscosity solutions to standard semilinear parabolic PDEs. 
The paper is organized as follows. In Sect. 2 we develop functional Itô calculus via regularization following [9]: after a brief introduction on finite dimensional stochastic calculus via regularization in Sect. 2.1 , we introduce and study the space $\mathscr{C}([-T, 0])$ in Sect.2.2; then, we define the pathwise derivatives and we prove the functional Itô's formula in Sect. 2.3; in Sect. 2.4, instead, we discuss the relation between functional Itô calculus via regularization and Banach space valued stochastic calculus for window processes. In Sect. 3, on the other hand, we study path-dependent PDEs following [10]. More precisely, in Sect. 3.1 we discuss strict solutions; in Sect. 3.2 we present a significant hedging example to motivate the introduction of a weaker notion of solution; finally, in Sect. 3.3 we provide the definition of strong-viscosity solution.

\section{Functional Itô Calculus: A Regularization Approach}

\subsection{Background: Finite Dimensional Calculus via Regularization}

The theory of stochastic calculus via regularization has been developed in several papers, starting from [37, 38]. We recall below only the results used in the present paper, and we refer to [40] for a survey on the subject. We emphasize that integrands are allowed to be anticipating. Moreover, the integration theory and calculus appear to be close to a pure pathwise approach even though there is still a probability space behind.

Fix a probability space $(\Omega, \mathscr{F}, \mathbb{P})$ and $T \in] 0, \infty\left[\right.$. Let $\mathbb{F}=\left(\mathscr{F}_{t}\right)_{t \in[0, T]}$ denote a filtration satisfying the usual conditions. Let $X=\left(X_{t}\right)_{t \in[0, T]}\left(\right.$ resp. $\left.Y=\left(Y_{t}\right)_{t \in[0, T]}\right)$ be a real continuous (resp. $\mathbb{P}$-a.s. integrable) process. Every real continuous process $X=\left(X_{t}\right)_{t \in[0, T]}$ is naturally extended to all $t \in \mathbb{R}$ setting $X_{t}=X_{0}, t \leq 0$, and $X_{t}=X_{T}, t \geq T$. We also define a $C([-T, 0])$-valued process $\mathbb{X}=\left(\mathbb{X}_{t}\right)_{t \in \mathbb{R}}$, called the window process associated with $X$, defined by

$$
\mathbb{X}_{t}:=\left\{X_{t+x}, x \in[-T, 0]\right\}, \quad t \in \mathbb{R} .
$$

This corresponds to the so-called segment process which appears for instance in [43].

Definition 1 Suppose that, for every $t \in[0, T]$, the limit

$$
\int_{0}^{t} Y_{s} d^{-} X_{s}:=\lim _{\varepsilon \rightarrow 0^{+}} \int_{0}^{t} Y_{s} \frac{X_{s+\varepsilon}-X_{s}}{\varepsilon} d s,
$$

exists in probability. If the obtained random function admits a continuous modification, that process is denoted by $\int_{0}^{\cdot} Y d^{-} X$ and called forward integral of $Y$ with respect to $X$. 
Definition 2 A family of processes $\left(H_{t}^{(\varepsilon)}\right)_{t \in[0, T]}$ is said to converge to $\left(H_{t}\right)_{t \in[0, T]}$ in the ucp sense, if $\sup _{0 \leq t \leq T}\left|H_{t}^{(\varepsilon)}-H_{t}\right|$ goes to 0 in probability, as $\varepsilon \rightarrow 0^{+}$.

Proposition 1 Suppose that the limit (3) exists in the ucp sense. Then, the forward integral $\int_{0}^{*} Y d^{-} X$ of $Y$ with respect to $X$ exists.

Let us introduce the concept of covariation, which is a crucial notion in stochastic calculus via regularization. Let us suppose that $X, Y$ are continuous processes.

Definition 3 The covariation of $X$ and $Y$ is defined by

$$
[X, Y]_{t}=[Y, X]_{t}=\lim _{\varepsilon \rightarrow 0^{+}} \frac{1}{\varepsilon} \int_{0}^{t}\left(X_{s+\varepsilon}-X_{s}\right)\left(Y_{s+\varepsilon}-Y_{s}\right) d s, \quad t \in[0, T],
$$

if the limit exists in probability for every $t \in[0, T]$, provided that the limiting random function admits a continuous version (this is the case if the limit holds in the ucp sense). If $X=Y, X$ is said to be a finite quadratic variation process and we set $[X]:=[X, X]$.

The forward integral and the covariation generalize the classical Itô integral and covariation for semimartingales. In particular, we have the following result, for a proof we refer to, e.g., [40].

Proposition 2 The following properties hold.

(i) Let $S^{1}, S^{2}$ be continuous $\mathbb{F}$-semimartingales. Then, $\left[S^{1}, S^{2}\right]$ is the classical bracket $\left[S^{1}, S^{2}\right]=\left\langle M^{1}, M^{2}\right\rangle$, where $M^{1}$ (resp. $M^{2}$ ) is the local martingale part of $S^{1}$ (resp. $\left.S^{2}\right)$.

(ii) Let $V$ be a continuous bounded variation process and $Y$ be a càdlàg process (or vice-versa); then $[V]=[Y, V]=0$. Moreover $\int_{0}^{\cdot} Y d^{-} V=\int_{0}^{\cdot} Y d V$, is the Lebesgue-Stieltjes integral.

(iii) If $W$ is a Brownian motion and $Y$ is an $\mathbb{F}$-progressively measurable process such that $\int_{0}^{T} Y_{s}^{2} d s<\infty, \mathbb{P}$-a.s., then $\int_{0}^{\cdot} Y d^{-} W$ exists and equals the Itô integral $\int_{0}^{\cdot} Y d W$.

We could have defined the forward integral using limits of non-anticipating Riemann sums. Another reason to use the regularization procedure is due to the fact that it extends the Itô integral, as Proposition 2(iii) shows. If the integrand had uncountable jumps (as $Y$ being the indicator function of the rational number in $[0,1]$ ) then, the Itô integral $\int_{0}^{*} Y d W$ would be zero $Y=0$ a.e. The limit of Riemann sums $\sum_{i} Y_{t_{i}}\left(W_{t_{i+1}}-W_{t_{i}}\right)$ would heavily depend on the discretization grid.

We end this crash introduction to finite dimensional stochastic calculus via regularization presenting one of its cornerstones: Itô's formula. It is a well-known result in the theory of semimartingales, but it also extends to the framework of finite quadratic variation processes. For a proof we refer to Theorem 2.1 of [39]. 
Theorem 1 Let $F:[0, T] \times \mathbb{R} \longrightarrow \mathbb{R}$ be of class $C^{1,2}([0, T] \times \mathbb{R})$ and $X=$ $\left(X_{t}\right)_{t \in[0, T]}$ be a real continuous finite quadratic variation process. Then, the following Itô's formula holds, $\mathbb{P}$-a.s.,

$$
\begin{array}{rlrl}
F\left(t, X_{t}\right)= & F\left(0, X_{0}\right)+\int_{0}^{t} \partial_{t} F\left(s, X_{s}\right) d s+\int_{0}^{t} \partial_{x} F\left(s, X_{s}\right) d^{-} X_{s} \\
& +\frac{1}{2} \int_{0}^{t} \partial_{x x}^{2} F\left(s, X_{s}\right) d[X]_{s}, & 0 \leq t \leq T .
\end{array}
$$

\subsubsection{The Deterministic Calculus via Regularization}

A useful particular case of finite dimensional stochastic calculus via regularization arises when $\Omega$ is a singleton, i.e., when the calculus becomes deterministic. In addition, in this deterministic framework we will make use of the definite integral on an interval $[a, b]$, where $a<b$ are two real numbers. Typically, we will consider $a=-T$ or $a=-t$ and $b=0$.

We start with two conventions. By default, every bounded variation function $f:[a, b] \rightarrow \mathbb{R}$ will be considered as càdlàg. Moreover, given a function $f:[a, b] \rightarrow$ $\mathbb{R}$, we will consider the following two extensions of $f$ to the entire real line:

$$
f_{J}(x):=\left\{\begin{array}{ll}
0, & x>b, \\
f(x), & x \in[a, b], \\
f(a), & x<a,
\end{array} \quad f_{\bar{J}}(x):= \begin{cases}f(b), & x>b, \\
f(x), & x \in[a, b], \\
0, & x<a,\end{cases}\right.
$$

where $J:=] a, b]$ and $\bar{J}=[a, b[$.

Definition 4 Let $f, g:[a, b] \rightarrow \mathbb{R}$ be càdlàg functions.

(i) Suppose that the following limit

$$
\int_{[a, b]} g(s) d^{-} f(s):=\lim _{\varepsilon \rightarrow 0^{+}} \int_{\mathbb{R}} g_{J}(s) \frac{f_{\bar{J}}(s+\varepsilon)-f_{\bar{J}}(s)}{\varepsilon} d s,
$$

exists and it is finite. Then, the obtained quantity is denoted by $\int_{[a, b]} g d^{-} f$ and called (deterministic, definite) forward integral of $g$ with respect to $f($ on $[a, b])$.

(ii) Suppose that the following limit

$$
\int_{[a, b]} g(s) d^{+} f(s):=\lim _{\varepsilon \rightarrow 0^{+}} \int_{\mathbb{R}} g_{J}(s) \frac{f_{\bar{J}}(s)-f_{\bar{J}}(s-\varepsilon)}{\varepsilon} d s,
$$

exists and it is finite. Then, the obtained quantity is denoted by $\int_{[a, b]} g d^{+} f$ and called (deterministic, definite) backward integral of $g$ with respect to $f$ (on $[a, b]$ ). 
The notation concerning this integral is justified since when the integrator $f$ has bounded variation the previous integrals are Lebesgue-Stieltjes integrals on $[a, b]$.

Proposition 3 Suppose $f:[a, b] \rightarrow \mathbb{R}$ with bounded variation and $g:[a, b] \rightarrow \mathbb{R}$ càdlàg. Then, we have

$$
\begin{aligned}
& \int_{[a, b]} g(s) d^{-} f(s)=\int_{[a, b]} g\left(s^{-}\right) d f(s):=g(a) f(a)+\int_{] a, b]} g\left(s^{-}\right) d f(s), \\
& \int_{[a, b]} g(s) d^{+} f(s)=\int_{[a, b]} g(s) d f(s):=g(a) f(a)+\int_{] a, b]} g(s) d f(s) .
\end{aligned}
$$

Proof Identity (7). We have

$$
\begin{aligned}
\int_{\mathbb{R}} g_{J}(s) \frac{f_{\bar{J}}(s+\varepsilon)-f_{\bar{J}}(s)}{\varepsilon} d s= & \frac{1}{\varepsilon} g(a) \int_{a-\varepsilon}^{a} f(s+\varepsilon) d s \\
& +\int_{a}^{b} g(s) \frac{f((s+\varepsilon) \wedge b)-f(s)}{\varepsilon} d s .
\end{aligned}
$$

The second integral on the right-hand side of (9) gives, by Fubini's theorem,

$$
\begin{aligned}
\int_{a}^{b} g(s)\left(\frac{1}{\varepsilon} \int_{] s,(s+\varepsilon) \wedge b]} d f(y)\right) d s & =\int_{] a, b]}\left(\frac{1}{\varepsilon} \int_{[a \vee(y-\varepsilon), y]} g(s) d s\right) d f(y) \\
& \stackrel{\varepsilon \rightarrow 0^{+}}{\longrightarrow} \int_{] a, b]} g\left(y^{-}\right) d f(y) .
\end{aligned}
$$

The first integral on the right-hand side of (9) goes to $g(a) f(a)$ as $\varepsilon \rightarrow 0^{+}$, so the result follows.

Identity (8). We have

$$
\begin{aligned}
\int_{\mathbb{R}} g_{J}(s) \frac{f_{\bar{J}}(s)-f_{\bar{J}}(s-\varepsilon)}{\varepsilon} d s= & \int_{a+\varepsilon}^{b} g(s) \frac{f(s)-f(s-\varepsilon)}{\varepsilon} d s \\
& +\frac{1}{\varepsilon} \int_{a}^{a+\varepsilon} g(s) f(s) d s .
\end{aligned}
$$

The second integral on the right-hand side of (10) goes to $g(a) f(a)$ as $\varepsilon \rightarrow 0^{+}$. The first one equals

$$
\int_{a+\varepsilon}^{b} g(s)\left(\frac{1}{\varepsilon} \int_{] s-\varepsilon, s]} d f(y)\right) d s=\int_{] a, b]}\left(\frac{1}{\varepsilon} \int_{] y,(y+\varepsilon) \wedge b]} g(s) d s\right) d f(y) \stackrel{\varepsilon \rightarrow 0^{+}}{\longrightarrow} \int_{] a, b]} g(y) d f(y),
$$

from which the claim follows. 
Let us now introduce the deterministic covariation.

Definition 5 Let $f, g:[a, b] \rightarrow \mathbb{R}$ be continuous functions and suppose that $0 \in$ $[a, b]$. The (deterministic) covariation of $f$ and $g($ on $[a, b])$ is defined by

$$
[f, g](x)=[g, f](x)=\lim _{\varepsilon \rightarrow 0^{+}} \frac{1}{\varepsilon} \int_{0}^{x}(f(s+\varepsilon)-f(s))(g(s+\varepsilon)-g(s)) d s, \quad x \in[a, b],
$$

if the limit exists and it is finite for every $x \in[a, b]$. If $f=g$, we set $[f]:=[f, f]$ and it is called quadratic variation of $f($ on $[a, b])$.

We notice that in Definition 5 the quadratic variation $[f]$ is continuous on $[a, b]$, since $f$ is a continuous function.

Remark 1 Notice that if $f$ is a fixed Brownian path and $g(s)=\varphi(s, f(s))$, with $\varphi \in C^{1}([a, b] \times \mathbb{R})$. Then $\int_{[a, b]} g(s) d^{-} f(s)$ exists for almost all (with respect to the Wiener measure on $C([a, b]))$ Brownian paths $f$. This latter result can be shown using Theorem 2.1 in [26] (which implies that the deterministic bracket exists, for almost all Brownian paths $f$, and $[f](s)=s$ ) and then applying Itô's formula in Theorem 1 above, with $\mathbb{P}$ given by the Dirac delta at a Brownian path $f$.

We conclude this subsection with an integration by parts formula for the deterministic forward and backward integrals.

Proposition 4 Let $f:[a, b] \rightarrow \mathbb{R}$ be a càdlàg function and $g:[a, b] \rightarrow \mathbb{R}$ be $a$ bounded variation function. Then, the following integration by parts formulae hold:

$$
\begin{gathered}
\int_{[a, b]} g(s) d^{-} f(s)=g(b) f(b)-\int_{] a, b]} f(s) d g(s), \\
\int_{[a, b]} g(s) d^{+} f(s)=g(b) f\left(b^{-}\right)-\int_{] a, b]} f\left(s^{-}\right) d g(s) .
\end{gathered}
$$

Proof Identity (11). The left-hand side of (11) is the limit, when $\varepsilon \rightarrow 0^{+}$, of

$$
\frac{1}{\varepsilon} \int_{a}^{b-\varepsilon} g(s) f(s+\varepsilon) d s-\frac{1}{\varepsilon} \int_{a}^{b} g(s) f(s) d s+\frac{1}{\varepsilon} \int_{b-\varepsilon}^{b} g(s) f(b) d s+\frac{1}{\varepsilon} \int_{a-\varepsilon}^{a} g(a) f(s+\varepsilon) d s .
$$

This gives

$$
\begin{aligned}
& \frac{1}{\varepsilon} \int_{a+\varepsilon}^{b} g(s-\varepsilon) f(s) d s-\frac{1}{\varepsilon} \int_{a}^{b} g(s) f(s) d s+\frac{1}{\varepsilon} \int_{b-\varepsilon}^{b} g(s) f(b) d s+\frac{1}{\varepsilon} \int_{a-\varepsilon}^{a} g(a) f(s+\varepsilon) d s \\
& =-\int_{a+\varepsilon}^{b} \frac{g(s)-g(s-\varepsilon)}{\varepsilon} f(s) d s-\frac{1}{\varepsilon} \int_{a}^{a+\varepsilon} g(s) f(s) d s+\frac{1}{\varepsilon} \int_{b-\varepsilon}^{b} g(s) f(b) d s \\
& \quad+\frac{1}{\varepsilon} \int_{a-\varepsilon}^{a} g(a) f(s+\varepsilon) d s .
\end{aligned}
$$


We see that

$$
\begin{array}{r}
\frac{1}{\varepsilon} \int_{b-\varepsilon}^{b} g(s) f(b) d s \stackrel{\varepsilon \rightarrow 0^{+}}{\longrightarrow} g\left(b^{-}\right) f(b), \\
\frac{1}{\varepsilon} \int_{a-\varepsilon}^{a} g(a) f(s+\varepsilon) d s-\frac{1}{\varepsilon} \int_{a}^{a+\varepsilon} g(s) f(s) d s \stackrel{\varepsilon \rightarrow 0^{+}}{\longrightarrow} 0 .
\end{array}
$$

Moreover, we have

$$
\begin{aligned}
-\int_{a+\varepsilon}^{b} \frac{g(s)-g(s-\varepsilon)}{\varepsilon} f(s) d s & =-\int_{a+\varepsilon}^{b} d s f(s) \frac{1}{\varepsilon} \int_{] s-\varepsilon, s]} d g(y) \\
& =-\int_{] a, b]} d g(y) \frac{1}{\varepsilon} \int_{y \vee(a+\varepsilon)}^{b \wedge(y+\varepsilon)} f(s) d s \stackrel{\varepsilon \rightarrow 0^{+}}{\longrightarrow}-\int_{] a, b[} d g(y) f(y) .
\end{aligned}
$$

In conclusion, we find

$$
\begin{aligned}
\int_{[a, b]} g(s) d^{-} f(s) & =-\int_{] a, b]} d g(y) f(y)+\left(g(b)-g\left(b^{-}\right)\right) f(b)+g\left(b^{-}\right) f(b) \\
& =-\int_{] a, b]} d g(y) f(y)+g(b) f(b)
\end{aligned}
$$

Identity (12). The left-hand side of (12) is given by the limit, as $\varepsilon \rightarrow 0^{+}$, of

$$
\begin{array}{r}
\frac{1}{\varepsilon} \int_{a}^{b} g(s) f(s) d s-\frac{1}{\varepsilon} \int_{a+\varepsilon}^{b} g(s) f(s-\varepsilon) d s=\frac{1}{\varepsilon} \int_{a}^{b} g(s) f(s) d s-\frac{1}{\varepsilon} \int_{a}^{b-\varepsilon} g(s+\varepsilon) f(s) d s \\
=-\int_{a}^{b-\varepsilon} f(s) \frac{g(s+\varepsilon)-g(s)}{\varepsilon} d s+\frac{1}{\varepsilon} \int_{b-\varepsilon}^{b} g(s) f(s) d s
\end{array}
$$

The second integral on the right-hand side goes to $g\left(b^{-}\right) f\left(b^{-}\right)$as $\varepsilon \rightarrow 0^{+}$. The first integral expression equals

$$
\begin{array}{r}
-\int_{\mathbb{R}} f_{J}(s) \frac{g \bar{J}^{-}(s+\varepsilon)-g \bar{J}(s)}{\varepsilon} d s+\frac{1}{\varepsilon} f(a) \int_{a-\varepsilon}^{a} g(s+\varepsilon) d s+\int_{b-\varepsilon}^{b} f(s) \frac{g(b)-g(s)}{\varepsilon} d s \\
\stackrel{\varepsilon \rightarrow 0^{+}}{\longrightarrow}-\int_{] a, b]} f\left(s^{-}\right) d g(s)-f(a) g(a)+f(a) g(a)+\left(g(b)-g\left(b^{-}\right)\right) f\left(b^{-}\right),
\end{array}
$$

taking into account identity (7). This gives us the result.

\subsection{The Spaces $\mathscr{C}([-T, 0])$ and $\mathscr{C}([-T, 0[)$}

Let $C([-T, 0])$ denote the set of real continuous functions on $[-T, 0]$, endowed with supremum norm $\|\eta\|_{\infty}=\sup _{x \in[-T, 0]}|\eta(x)|$, for any $\eta \in C([-T, 0])$. 
Remark 2 We shall develop functional Itô calculus via regularization firstly for timeindependent functionals $\mathscr{U}: C([-T, 0]) \rightarrow \mathbb{R}$, since we aim at emphasizing that in our framework the time variable and the path play two distinct roles, as emphasized in the introduction. This, also, allows us to focus only on the definition of horizontal and vertical derivatives. Clearly, everything can be extended in an obvious way to the time-dependent case $\mathscr{U}:[0, T] \times C([-T, 0]) \rightarrow \mathbb{R}$, as we shall illustrate later.

Consider a map $\mathscr{U}: C([-T, 0]) \rightarrow \mathbb{R}$. Our aim is to derive a functional Itô's formula for $\mathscr{U}$. To do this, we are led to define the functional (i.e., horizontal and vertical) derivatives for $\mathscr{U}$ in the spirit of $[5,17]$. Since the definition of functional derivatives necessitates of discontinuous paths, in [5] the idea is to consider functionals defined on the space of càdlàg trajectories $\mathbb{D}([-T, 0])$. However, we can not, in general, extend in a unique way a functional $\mathscr{U}$ defined on $C([-T, 0])$ to $\mathbb{D}([-T, 0])$. Our idea, instead, is to consider a larger space than $C([-T, 0])$, denoted by $\mathscr{C}([-T, 0])$, which is the space of bounded trajectories on $[-T, 0]$, continuous on $[-T, 0[$ and with possibly a jump at 0 . We endow $\mathscr{C}([-T, 0])$ with a (inductive) topology such that $C([-T, 0])$ is dense in $\mathscr{C}([-T, 0])$ with respect to this topology. Therefore, if $\mathscr{U}$ is continuous with respect to the topology of $\mathscr{C}([-T, 0])$, then it admits a unique continuous extension $u: \mathscr{C}([-T, 0]) \rightarrow \mathbb{R}$.

Definition 6 We denote by $\mathscr{C}([-T, 0])$ the set of bounded functions $\eta:[-T, 0]$ $\rightarrow \mathbb{R}$ such that $\eta$ is continuous on $[-T, 0[$, equipped with the topology we now describe.

Convergence We endow $\mathscr{C}([-T, 0])$ with a topology inducing the following convergence: $\left(\eta_{n}\right)_{n}$ converges to $\eta$ in $\mathscr{C}([-T, 0])$ as $n$ tends to infinity if the following holds.

(i) $\left\|\eta_{n}\right\|_{\infty} \leq C$, for any $n \in \mathbb{N}$, for some positive constant $C$ independent of $n$;

(ii) $\sup _{x \in K}\left|\eta_{n}(x)-\eta(x)\right| \rightarrow 0$ as $n$ tends to infinity, for any compact set $K \subset$ $[-T, 0[$

(iii) $\eta_{n}(0) \rightarrow \eta(0)$ as $n$ tends to infinity.

Topology For each compact $K \subset\left[-T, 0\left[\right.\right.$ define the seminorm $p_{K}$ on $\mathscr{C}([-T, 0])$ by

$$
p_{K}(\eta)=\sup _{x \in K}|\eta(x)|+|\eta(0)|, \quad \forall \eta \in \mathscr{C}([-T, 0]) .
$$

Let $M>0$ and $\mathscr{C}_{M}([-T, 0])$ be the set of functions in $\mathscr{C}([-T, 0])$ which are bounded by $M$. Still denote $p_{K}$ the restriction of $p_{K}$ to $\mathscr{C}_{M}([-T, 0])$ and consider the topology on $\mathscr{C}_{M}([-T, 0])$ induced by the collection of seminorms $\left(p_{K}\right)_{K}$. Then, we endow $\mathscr{C}([-T, 0])$ with the smallest topology (inductive topology) turning all the inclusions $i_{M}: \mathscr{C}_{M}([-T, 0]) \rightarrow \mathscr{C}([-T, 0])$ into continuous maps. 
Remark 3 (i) Notice that $C([-T, 0])$ is dense in $\mathscr{C}([-T, 0])$, when endowed with the topology of $\mathscr{C}([-T, 0])$. As a matter of fact, let $\eta \in \mathscr{C}([-T, 0])$ and define, for any $n \in \mathbb{N} \backslash\{0\}$,

$$
\varphi_{n}(x)= \begin{cases}\eta(x), & -T \leq x \leq-1 / n \\ n(\eta(0)-\eta(-1 / n)) x+\eta(0), & -1 / n<x \leq 0\end{cases}
$$

Then, we see that $\varphi_{n} \in C([-T, 0])$ and $\varphi_{n} \rightarrow \eta$ in $\mathscr{C}([-T, 0])$.

Now, for any $a \in \mathbb{R}$ define

$$
\begin{aligned}
& C_{a}([-T, 0]):=\{\eta \in C([-T, 0]): \eta(0)=a\}, \\
& \mathscr{C}_{a}([-T, 0]):=\{\eta \in \mathscr{C}([-T, 0]): \eta(0)=a\} .
\end{aligned}
$$

Then, $C_{a}([-T, 0])$ is dense in $\mathscr{C}_{a}([-T, 0])$ with respect to the topology of $\mathscr{C}([-T, 0])$.

(ii) We provide two examples of functionals $\mathscr{U}: C([-T, 0]) \rightarrow \mathbb{R}$, continuous with respect to the topology of $\mathscr{C}([-T, 0])$, and necessarily with respect to the topology of $C([-T, 0])$ (the proof is straightforward and not reported):

(a) $\mathscr{U}(\eta)=g\left(\eta\left(t_{1}\right), \ldots, \eta\left(t_{n}\right)\right)$, for all $\eta \in C([-T, 0])$, with $-T \leq t_{1}<\cdots<$ $t_{n} \leq 0$ and $g: \mathbb{R}^{n} \rightarrow \mathbb{R}$ continuous.

(b) $\mathscr{U}(\eta)=\int_{[-T, 0]} \varphi(x) d^{-} \eta(x)$, for all $\eta \in C([-T, 0])$, with $\varphi:[0, T] \rightarrow \mathbb{R}$ a càdlàg bounded variation function. Concerning this example, keep in mind that, using the integration by parts formula, $\mathscr{U}(\eta)$ admits the representation (11).

(iii) Consider the functional $\mathscr{U}(\eta)=\sup _{x \in[-T, 0]} \eta(x)$, for all $\eta \in C([-T, 0])$. It is obviously continuous, but it is not continuous with respect to the topology of $\mathscr{C}([-T, 0])$. As a matter of fact, for any $n \in \mathbb{N}$ consider $\eta_{n} \in C([-T, 0])$ given by

$$
\eta_{n}(x)= \begin{cases}0, & -T \leq x \leq-\frac{T}{2^{n}}, \\ \frac{2^{n+1}}{T} x+2, & -\frac{T}{2^{n}}<x \leq-\frac{T}{2^{n+1}}, \\ -\frac{2^{n+1}}{T} x, & -\frac{T}{2^{n+1}}<x \leq 0 .\end{cases}
$$

Then, $\mathscr{U}\left(\eta_{n}\right)=\sup _{x \in[-T, 0]} \eta_{n}(x)=1$, for any $n$. However, $\eta_{n}$ converges to the zero function in $\mathscr{C}([-T, 0])$, as $n$ tends to infinity. This example will play an important role in Sect. 3 to justify a weaker notion of solution to the path-dependent semilinear Kolmogorov equation.

To define the functional derivatives, we shall need to separate the "past" from the "present" of $\eta \in \mathscr{C}([-T, 0])$. Indeed, roughly speaking, the horizontal derivative calls in the past values of $\eta$, namely $\{\eta(x): x \in[-T, 0[\}$, while the vertical derivative calls in the present value of $\eta$, namely $\eta(0)$. To this end, it is useful to introduce the space $\mathscr{C}([-T, 0[)$. 
Definition 7 We denote by $\mathscr{C}([-T, 0[)$ the set of bounded continuous functions $\gamma:[-T, 0[\rightarrow \mathbb{R}$, equipped with the topology we now describe.

Convergence We endow $\mathscr{C}([-T, 0[)$ with a topology inducing the following convergence: $\left(\gamma_{n}\right)_{n}$ converges to $\gamma$ in $\mathscr{C}([-T, 0[)$ as $n$ tends to infinity if:

(i) $\sup _{x \in[-T, 0[}\left|\gamma_{n}(x)\right| \leq C$, for any $n \in \mathbb{N}$, for some positive constant $C$ independent of $n$;

(ii) $\sup _{x \in K}\left|\gamma_{n}(x)-\gamma(x)\right| \rightarrow 0$ as $n$ tends to infinity, for any compact set $K \subset$ $[-T, 0[$.

Topology For each compact $K \subset\left[-T, 0\left[\right.\right.$ define the seminorm $q_{K}$ on $\mathscr{C}([-T, 0[)$ by

$$
q_{K}(\gamma)=\sup _{x \in K}|\gamma(x)|, \quad \forall \gamma \in \mathscr{C}([-T, 0[)
$$

Let $M>0$ and $\mathscr{C}_{M}([-T, 0[)$ be the set of functions in $\mathscr{C}([-T, 0[)$ which are bounded by $M$. Still denote $q_{K}$ the restriction of $q_{K}$ to $\mathscr{C}_{M}([-T, 0[)$ and consider the topology on $\mathscr{C}_{M}\left(\left[-T, 0[)\right.\right.$ induced by the collection of seminorms $\left(q_{K}\right)_{K}$. Then, we endow $\mathscr{C}([-T, 0[)$ with the smallest topology (inductive topology) turning all the inclusions $i_{M}: \mathscr{C}_{M}([-T, 0[) \rightarrow \mathscr{C}([-T, 0[)$ into continuous maps.

Remark 4 (i) Notice that $\mathscr{C}([-T, 0])$ is isomorphic to $\mathscr{C}([-T, 0[) \times \mathbb{R}$. As a matter of fact, it is enough to consider the map

$$
\begin{aligned}
J: \mathscr{C}([-T, 0]) & \rightarrow \mathscr{C}([-T, 0[) \times \mathbb{R} \\
\eta & \mapsto\left(\eta_{\mid[-T, 0[, \eta(0)) .}\right.
\end{aligned}
$$

Observe that $J^{-1}: \mathscr{C}\left(\left[-T, 0[) \times \mathbb{R} \rightarrow \mathscr{C}([-T, 0])\right.\right.$ is given by $J^{-1}(\gamma, a)=$ $\gamma 1_{[-T, 0[}+a 1_{\{0\}}$.

(ii) $\mathscr{C}([-T, 0])$ is a space which contains $C([-T, 0])$ as a dense subset and it has the property of separating "past" from "present". Another space having the same property is $L^{2}([-T, 0] ; d \mu)$ where $\mu$ is the sum of the Dirac measure at zero and Lebesgue measure. Similarly as for item (i), that space is isomorphic to $L^{2}([-T, 0]) \times \mathbb{R}$, which is a very popular space appearing in the analysis of functional dependent (as delay) equations, starting from [4].

For every $u: \mathscr{C}([-T, 0]) \rightarrow \mathbb{R}$, we can now exploit the space $\mathscr{C}([-T, 0[)$ to define a map $\tilde{u}: \mathscr{C}([-T, 0[) \times \mathbb{R} \rightarrow \mathbb{R}$ where "past" and "present" are separated.

Definition 8 Let $u: \mathscr{C}([-T, 0]) \rightarrow \mathbb{R}$ and define $\tilde{u}: \mathscr{C}([-T, 0[) \times \mathbb{R} \rightarrow \mathbb{R}$ as

$$
\tilde{u}(\gamma, a):=u\left(\gamma 1_{[-T, 0[}+a 1_{\{0\}}\right), \quad \forall(\gamma, a) \in \mathscr{C}([-T, 0[) \times \mathbb{R} .
$$

In particular, we have $u(\eta)=\tilde{u}\left(\eta_{\mid[-T, 0[}, \eta(0)\right)$, for all $\eta \in \mathscr{C}([-T, 0])$. 
We conclude this subsection with a characterization of the dual spaces of $\mathscr{C}([-T, 0])$ and $\mathscr{C}([-T, 0[)$, which has an independent interest. Firstly, we need to introduce the set $\mathscr{M}([-T, 0])$ of finite signed Borel measures on $[-T, 0]$. We also denote $\mathscr{M}_{0}([-T, 0]) \subset \mathscr{M}([-T, 0])$ the set of measures $\mu$ such that $\mu(\{0\})=0$.

Proposition 5 Let $\Lambda \in \mathscr{C}([-T, 0])^{*}$, the dual space of $\mathscr{C}([-T, 0])$. Then, there exists a unique $\mu \in \mathscr{M}([-T, 0])$ such that

$$
\Lambda \eta=\int_{[-T, 0]} \eta(x) \mu(d x), \quad \forall \eta \in \mathscr{C}([-T, 0]) .
$$

Proof Let $\Lambda \in \mathscr{C}([-T, 0])^{*}$ and define

$$
\tilde{\Lambda} \varphi:=\Lambda \varphi, \quad \forall \varphi \in C([-T, 0]) .
$$

Notice that $\tilde{\Lambda}: C([-T, 0]) \rightarrow \mathbb{R}$ is a continuous functional on the Banach space $C([-T, 0])$ endowed with the supremum norm $\|\cdot\|_{\infty}$. Therefore $\tilde{\Lambda} \in C([-T, 0])^{*}$ and it follows from Riesz representation theorem (see, e.g., Theorem 6.19 in [36]) that there exists a unique $\mu \in \mathscr{M}([-T, 0])$ such that

$$
\tilde{\Lambda} \varphi=\int_{[-T, 0]} \varphi(x) \mu(d x), \quad \forall \varphi \in C([-T, 0]) .
$$

Obviously $\tilde{\Lambda}$ is also continuous with respect to the topology of $\mathscr{C}([-T, 0])$. Since $C([-T, 0])$ is dense in $\mathscr{C}([-T, 0])$ with respect to the topology of $\mathscr{C}([-T, 0])$, we deduce that there exists a unique continuous extension of $\tilde{\Lambda}$ to $\mathscr{C}([-T, 0])$, which is clearly given by

$$
\Lambda \eta=\int_{[-T, 0]} \eta(x) \mu(d x), \quad \forall \eta \in \mathscr{C}([-T, 0]) .
$$

Proposition 6 Let $\Lambda \in \mathscr{C}\left(\left[-T, 0[)^{*}\right.\right.$, the dual space of $\mathscr{C}([-T, 0[)$. Then, there exists a unique $\mu \in \mathscr{M}_{0}([-T, 0])$ such that

$$
\Lambda \gamma=\int_{[-T, 0[} \gamma(x) \mu(d x), \quad \forall \gamma \in \mathscr{C}([-T, 0[) .
$$

Proof Let $\Lambda \in \mathscr{C}\left(\left[-T, 0[)^{*}\right.\right.$ and define

$$
\tilde{\Lambda} \eta:=\Lambda\left(\eta_{\mid[-T, 0[}\right), \quad \forall \eta \in \mathscr{C}([-T, 0]) .
$$


Notice that $\tilde{\Lambda}: \mathscr{C}([-T, 0]) \rightarrow \mathbb{R}$ is a continuous functional on $\mathscr{C}([-T, 0])$. It follows from Proposition 5 that there exists a unique $\mu \in \mathscr{M}([-T, 0])$ such that

$$
\tilde{\Lambda} \eta=\int_{[-T, 0]} \eta(x) \mu(d x)=\int_{[-T, 0[} \eta(x) \mu(d x)+\eta(0) \mu(\{0\}), \quad \forall \eta \in \mathscr{C}([-T, 0]) .
$$

Let $\eta_{1}, \eta_{2} \in \mathscr{C}([-T, 0])$ be such that $\eta_{1} 1_{[-T, 0[}=\eta_{2} 1_{[-T, 0[}$. Then, we see from (14) that $\tilde{\Lambda} \eta_{1}=\tilde{\Lambda} \eta_{2}$, which in turn implies from (15) that $\mu(\{0\})=0$. In conclusion, $\mu \in \mathscr{M}_{0}([-T, 0])$ and $\Lambda$ is given by

$$
\Lambda \gamma=\int_{[-T, 0[} \gamma(x) \mu(d x), \quad \forall \gamma \in \mathscr{C}([-T, 0[) .
$$

\subsection{Functional Derivatives and Functional Itô's Formula}

In the present section we shall prove one of the main result of this section, namely the functional Itô's formula for $\mathscr{U}: C([-T, 0]) \rightarrow \mathbb{R}$ and, more generally, for $\mathscr{U}:[0, T] \times C([-T, 0]) \rightarrow \mathbb{R}$. We begin introducing the functional derivatives, firstly for a functional $u: \mathscr{C}([-T, 0]) \rightarrow \mathbb{R}$, and then for $\mathscr{U}: C([-T, 0]) \rightarrow \mathbb{R}$.

Definition 9 Consider $u: \mathscr{C}([-T, 0]) \rightarrow \mathbb{R}$ and $\eta \in \mathscr{C}([-T, 0])$.

(i) We say that $u$ admits the horizontal derivative at $\eta$ if the following limit exists and it is finite:

$$
D^{H} u(\eta):=\lim _{\varepsilon \rightarrow 0^{+}} \frac{u\left(\eta(\cdot) 1_{[-T, 0[}+\eta(0) 1_{\{0\}}\right)-u\left(\eta(\cdot-\varepsilon) 1_{[-T, 0[}+\eta(0) 1_{\{0\}}\right)}{\varepsilon} .
$$

(i) 'Let $\tilde{u}$ be as in (13), then we say that $\tilde{u}$ admits the horizontal derivative at $(\gamma, a) \in \mathscr{C}([-T, 0[) \times \mathbb{R}$ if the following limit exists and it is finite:

$$
D^{H} \tilde{u}(\gamma, a):=\lim _{\varepsilon \rightarrow 0^{+}} \frac{\tilde{u}(\gamma(\cdot), a)-\tilde{u}(\gamma(\cdot-\varepsilon), a)}{\varepsilon} .
$$

Notice that if $D^{H} u(\eta)$ exists then $D^{H} \tilde{u}\left(\eta_{\mid[-T, 0[}, \eta(0)\right)$ exists and they are equal; viceversa, if $D^{H} \tilde{u}(\gamma, a)$ exists then $D^{H} u\left(\gamma 1_{[-T, 0[}+a 1_{\{0\}}\right)$ exists and they are equal. (ii) We say that $u$ admits the first-order vertical derivative at $\eta$ if the first-order partial derivative $\partial_{a} \tilde{u}\left(\eta_{\mid[-T, 0[}, \eta(0)\right)$ at $\left(\eta_{\mid[-T, 0[}, \eta(0)\right)$ of $\tilde{u}$ with respect to its second argument exists and we set

$$
D^{V} u(\eta):=\partial_{a} \tilde{u}\left(\eta_{\mid[-T, 0[}, \eta(0)\right)
$$


(iii) We say that $u$ admits the second-order vertical derivative at $\eta$ if the secondorder partial derivative at $\left(\eta_{\mid[-T, 0[}, \eta(0)\right)$ of $\tilde{u}$ with respect to its second argument, denoted by $\partial_{a a}^{2} \tilde{u}\left(\eta_{\mid[-T, 0[}, \eta(0)\right)$, exists and we set

$$
D^{V V} u(\eta):=\partial_{a a}^{2} \tilde{u}\left(\eta_{\mid[-T, 0[}, \eta(0)\right)
$$

Definition 10 We say that $u: \mathscr{C}([-T, 0]) \rightarrow \mathbb{R}$ is of class $\mathscr{C}^{1,2}$ (past $\times$ present) if

(i) $u$ is continuous;

(ii) $D^{H} u$ exists everywhere on $\mathscr{C}([-T, 0])$ and for every $\gamma \in \mathscr{C}([-T, 0[)$ the map

$$
(\varepsilon, a) \longmapsto D^{H} \tilde{u}(\gamma(\cdot-\varepsilon), a), \quad(\varepsilon, a) \in[0, \infty[\times \mathbb{R}
$$

is continuous on $[0, \infty[\times \mathbb{R}$;

(iii) $D^{V} u$ and $D^{V V} u$ exist everywhere on $\mathscr{C}([-T, 0])$ and are continuous.

Remark 5 Notice that in Definition 10 we still obtain the same class of functions $\mathscr{C}^{1,2}$ (past $\times$ present) if we substitute point (ii) with

(ii') $D^{H} u$ exists everywhere on $\mathscr{C}([-T, 0])$ and for every $\gamma \in \mathscr{C}([-T, 0[)$ there exists $\delta(\gamma)>0$ such that the map

$$
(\varepsilon, a) \longmapsto D^{H} \tilde{u}(\gamma(\cdot-\varepsilon), a), \quad(\varepsilon, a) \in[0, \infty[\times \mathbb{R}
$$

is continuous on $[0, \delta(\gamma)[\times \mathbb{R}$.

In particular, if (ii') holds then we can always take $\delta(\gamma)=\infty$ for any $\gamma \in$ $\mathscr{C}([-T, 0[)$, which implies (ii). To prove this last statement, let us proceed by contradiction assuming that

$$
\delta^{*}(\gamma)=\sup \{\delta(\gamma)>0 \text { : the map (17) is continuous on }[0, \delta(\gamma)[\times \mathbb{R}\}<\infty \text {. }
$$

Notice that $\delta^{*}(\gamma)$ is in fact a max, therefore the map (18) is continuous on $\left[0, \delta^{*}(\gamma)\left[\times \mathbb{R}\right.\right.$. Now, define $\bar{\gamma}(\cdot):=\gamma\left(\cdot-\delta^{*}(\gamma)\right)$. Then, by condition (ii') there exists $\delta(\bar{\gamma})>0$ such that the map

$$
(\varepsilon, a) \longmapsto D^{H} \tilde{u}(\bar{\gamma}(\cdot-\varepsilon), a)=D^{H} \tilde{u}\left(\gamma\left(\cdot-\varepsilon-\delta^{*}(\gamma)\right), a\right)
$$

is continuous on $[0, \delta(\bar{\gamma})[\times \mathbb{R}$. This shows that the map (18) is continuous on $\left[0, \delta^{*}(\gamma)+\delta(\bar{\gamma})\left[\times \mathbb{R}\right.\right.$, a contradiction with the definition of $\delta^{*}(\gamma)$.

We can now provide the definition of functional derivatives for a map $\mathscr{U}: C([-T, 0])$ $\rightarrow \mathbb{R}$.

Definition 11 Let $\mathscr{U}: C([-T, 0]) \rightarrow \mathbb{R}$ and $\eta \in C([-T, 0])$. Suppose that there exists a unique extension $u: \mathscr{C}([-T, 0]) \rightarrow \mathbb{R}$ of $\mathscr{U}$ (e.g., if $\mathscr{U}$ is continuous with respect to the topology of $\mathscr{C}([-T, 0]))$. Then we define the following concepts. 
(i) The horizontal derivative of $\mathscr{U}$ at $\eta$ as:

$$
D^{H} \mathscr{U}(\eta):=D^{H} u(\eta) .
$$

(ii) The first-order vertical derivative of $\mathscr{U}$ at $\eta$ as:

$$
D^{V} \mathscr{U}(\eta):=D^{V} u(\eta)
$$

(iii) The second-order vertical derivative of $\mathscr{U}$ at $\eta$ as:

$$
D^{V V} \mathscr{U}(\eta):=D^{V V} u(\eta)
$$

Definition 12 We say that $\mathscr{U}: C([-T, 0]) \rightarrow \mathbb{R}$ is $C^{1,2}$ (past $\times$ present) if $\mathscr{U}$ admits a (necessarily unique) extension $u: \mathscr{C}([-T, 0]) \rightarrow \mathbb{R}$ of class $\mathscr{C}^{1,2}$ (past $\times$ present).

Theorem 2 Let $\mathscr{U}: C([-T, 0]) \rightarrow \mathbb{R}$ be of class $C^{1,2}$ (past $\times$ present $)$ and $X=\left(X_{t}\right)_{t \in[0, T]}$ be a real continuous finite quadratic variation process. Then, the following functional Itô's formula holds, $\mathbb{P}$-a.s.,

$$
\mathscr{U}\left(\mathbb{X}_{t}\right)=\mathscr{U}\left(\mathbb{X}_{0}\right)+\int_{0}^{t} D^{H} \mathscr{U}\left(\mathbb{X}_{s}\right) d s+\int_{0}^{t} D^{V} \mathscr{U}\left(\mathbb{X}_{s}\right) d^{-} X_{s}+\frac{1}{2} \int_{0}^{t} D^{V V} \mathscr{U}\left(\mathbb{X}_{s}\right) d[X]_{s},
$$

for all $0 \leq t \leq T$, where the window process $\mathbb{X}$ was defined in (2).

Proof Fix $t \in[0, T]$ and consider the quantity

$$
I_{0}(\varepsilon, t)=\int_{0}^{t} \frac{\mathscr{U}\left(\mathbb{X}_{s+\varepsilon}\right)-\mathscr{U}\left(\mathbb{X}_{s}\right)}{\varepsilon} d s=\frac{1}{\varepsilon} \int_{t}^{t+\varepsilon} \mathscr{U}\left(\mathbb{X}_{s}\right) d s-\frac{1}{\varepsilon} \int_{0}^{\varepsilon} \mathscr{U}\left(\mathbb{X}_{s}\right) d s, \quad \varepsilon>0 .
$$

Since the process $\left(\mathscr{U}\left(\mathbb{X}_{s}\right)\right)_{s \geq 0}$ is continuous, $I_{0}(\varepsilon, t)$ converges ucp to $\mathscr{U}\left(\mathbb{X}_{t}\right)$ $\mathscr{U}\left(\mathbb{X}_{0}\right)$, namely $\sup _{0 \leq t \leq T}\left|I_{0}(\varepsilon, t)-\left(\mathscr{U}\left(\mathbb{X}_{t}\right)-\mathscr{U}\left(\mathbb{X}_{0}\right)\right)\right|$ converges to zero in probability when $\varepsilon \rightarrow 0^{+}$. On the other hand, we can write $I_{0}(\varepsilon, t)$ in terms of the function $\tilde{u}$, defined in (13), as follows

$$
I_{0}(\varepsilon, t)=\int_{0}^{t} \frac{\tilde{u}\left(\mathbb{X}_{s+\varepsilon \mid[-T, 0[}, X_{s+\varepsilon}\right)-\tilde{u}\left(\mathbb{X}_{s \mid[-T, 0[}, X_{s}\right)}{\varepsilon} d s .
$$

Now we split $I_{0}(\varepsilon, t)$ into the sum of two terms

$$
\begin{aligned}
& I_{1}(\varepsilon, t)=\int_{0}^{t} \frac{\tilde{u}\left(\mathbb{X}_{s+\varepsilon \mid[-T, 0[}, X_{s+\varepsilon}\right)-\tilde{u}\left(\mathbb{X}_{s \mid[-T, 0[}, X_{s+\varepsilon}\right)}{\varepsilon} d s, \\
& I_{2}(\varepsilon, t)=\int_{0}^{t} \frac{\tilde{u}\left(\mathbb{X}_{s \mid[-T, 0[}, X_{s+\varepsilon}\right)-\tilde{u}\left(\mathbb{X}_{s \mid[-T, 0[}, X_{s}\right)}{\varepsilon} d s .
\end{aligned}
$$


We begin proving that

$$
I_{1}(\varepsilon, t) \underset{\varepsilon \rightarrow 0^{+}}{\stackrel{\mathrm{ucp}}{\longrightarrow}} \int_{0}^{t} D^{H} \mathscr{U}\left(\mathbb{X}_{s}\right) d s
$$

Firstly, fix $\gamma \in \mathscr{C}([-T, 0[)$ and define

$$
\phi(\varepsilon, a):=\tilde{u}(\gamma(\cdot-\varepsilon), a), \quad(\varepsilon, a) \in[0, \infty[\times \mathbb{R} .
$$

Then, denoting by $\partial_{\varepsilon}^{+} \phi$ the right partial derivative of $\phi$ with respect to $\varepsilon$ and using formula (17), we find

$$
\begin{aligned}
\partial_{\varepsilon}^{+} \phi(\varepsilon, a) & =\lim _{r \rightarrow 0^{+}} \frac{\phi(\varepsilon+r, a)-\phi(\varepsilon, a)}{r} \\
& =-\lim _{r \rightarrow 0^{+}} \frac{\tilde{u}(\gamma(\cdot-\varepsilon), a)-\tilde{u}(\gamma(\cdot-\varepsilon-r), a)}{r} \\
& =-D^{H} \tilde{u}(\gamma(\cdot-\varepsilon), a), \quad \forall(\varepsilon, a) \in[0, \infty[\times \mathbb{R} .
\end{aligned}
$$

Since $u \in \mathscr{C}^{1,2}$ (past $\times$ present), we see from Definition 10(ii), that $\partial_{\varepsilon}^{+} \phi$ is continuous on $[0, \infty[\times \mathbb{R}$. It follows from a standard differential calculus result (see for example Corollary 1.2 , Chap. 2 , in [32]) that $\phi$ is continuously differentiable on $[0, \infty[\times \mathbb{R}$ with respect to its first argument. Then, for every $(\varepsilon, a) \in[0, \infty[\times \mathbb{R}$, from the fundamental theorem of calculus, we have

$$
\phi(\varepsilon, a)-\phi(0, a)=\int_{0}^{\varepsilon} \partial_{\varepsilon} \phi(r, a) d r,
$$

which in terms of $\tilde{u}$ reads

$$
\tilde{u}(\gamma(\cdot), a)-\tilde{u}(\gamma(\cdot-\varepsilon), a)=\int_{0}^{\varepsilon} D^{H} \tilde{u}(\gamma(\cdot-r), a) d r .
$$

Now, we rewrite, by means of a shift in time, the term $I_{1}(\varepsilon, t)$ in $(20)$ as follows:

$$
\begin{aligned}
I_{1}(\varepsilon, t)= & \int_{0}^{t} \frac{\tilde{u}\left(\mathbb{X}_{S \mid[-T, 0[}, X_{S}\right)-\tilde{u}\left(\mathbb{X}_{S-\varepsilon \mid[-T, 0[}, X_{S}\right)}{\varepsilon} d s \\
& +\int_{t}^{t+\varepsilon} \frac{\tilde{u}\left(\mathbb{X}_{S \mid[-T, 0[}, X_{S}\right)-\tilde{u}\left(\mathbb{X}_{S-\varepsilon \mid[-T, 0[}, X_{S}\right)}{\varepsilon} d s \\
& -\int_{0}^{\varepsilon} \frac{\tilde{u}\left(\mathbb{X}_{S \mid[-T, 0[}, X_{S}\right)-\tilde{u}\left(\mathbb{X}_{S-\varepsilon \mid[-T, 0[}, X_{S}\right)}{\varepsilon} d s
\end{aligned}
$$

Plugging (23) into (24), setting $\gamma=\mathbb{X}_{s}, a=X_{s}$, we obtain 


$$
\begin{aligned}
I_{1}(\varepsilon, t)= & \int_{0}^{t} \frac{1}{\varepsilon}\left(\int_{0}^{\varepsilon} D^{H} \tilde{u}\left(\mathbb{X}_{s-r \mid[-T, 0[}, X_{s}\right) d r\right) d s \\
& +\int_{t}^{t+\varepsilon} \frac{1}{\varepsilon}\left(\int_{0}^{\varepsilon} D^{H} \tilde{u}\left(\mathbb{X}_{S-r \mid[-T, 0[}, X_{S}\right) d r\right) d s \\
& -\int_{0}^{\varepsilon} \frac{1}{\varepsilon}\left(\int_{0}^{\varepsilon} D^{H} \tilde{u}\left(\mathbb{X}_{S-r \mid[-T, 0[}, X_{S}\right) d r\right) d s
\end{aligned}
$$

Observe that

$$
\int_{0}^{t} \frac{1}{\varepsilon}\left(\int_{0}^{\varepsilon} D^{H} \tilde{u}\left(\mathbb{X}_{s-r \mid[-T, 0[}, X_{S}\right) d r\right) d s \underset{\varepsilon \rightarrow 0^{+}}{\stackrel{\mathrm{ucp}}{\longrightarrow}} \int_{0}^{t} D^{H} u\left(\mathbb{X}_{S}\right) d s .
$$

Similarly, we see that the other two terms in (25) converge ucp to zero. As a consequence, we get (22).

Regarding $I_{2}(\varepsilon, t)$ in $(21)$, it can be written, by means of the following standard Taylor's expansion for a function $f \in C^{2}(\mathbb{R})$ :

$$
\begin{aligned}
f(b)= & f(a)+f^{\prime}(a)(b-a)+\frac{1}{2} f^{\prime \prime}(a)(b-a)^{2} \\
& +\int_{0}^{1}(1-\alpha)\left(f^{\prime \prime}(a+\alpha(b-a))-f^{\prime \prime}(a)\right)(b-a)^{2} d \alpha,
\end{aligned}
$$

as the sum of the following three terms:

$$
\begin{aligned}
I_{21}(\varepsilon, t)= & \int_{0}^{t} \partial_{a} \tilde{u}\left(\mathbb{X}_{s \mid[-T, 0[}, X_{s}\right) \frac{X_{s+\varepsilon}-X_{s}}{\varepsilon} d s \\
I_{22}(\varepsilon, t)= & \frac{1}{2} \int_{0}^{t} \partial_{a a}^{2} \tilde{u}\left(\mathbb{X}_{s \mid[-T, 0[}, X_{s}\right) \frac{\left(X_{s+\varepsilon}-X_{s}\right)^{2}}{\varepsilon} d s \\
I_{23}(\varepsilon, t)= & \int_{0}^{t}\left(\int _ { 0 } ^ { 1 } ( 1 - \alpha ) \left(\partial_{a a}^{2} \tilde{u}\left(\mathbb{X}_{s \mid[-T, 0[}, X_{s}+\alpha\left(X_{s+\varepsilon}-X_{S}\right)\right)\right.\right. \\
& \left.\left.-\partial_{a a}^{2} \tilde{u}\left(\mathbb{X}_{S \mid[-T, 0[}, X_{S}\right)\right) \frac{\left(X_{s+\varepsilon}-X_{s}\right)^{2}}{\varepsilon} d \alpha\right) d s .
\end{aligned}
$$

By similar arguments as in Proposition 1.2 of [39], we have

$$
I_{22}(\varepsilon, t) \underset{\varepsilon \rightarrow 0^{+}}{\stackrel{\mathrm{ucp}}{\longrightarrow}} \frac{1}{2} \int_{0}^{t} \partial_{a a}^{2} \tilde{u}\left(\mathbb{X}_{s \mid[-T, 0[}, X_{S}\right) d[X]_{s}=\frac{1}{2} \int_{0}^{t} D^{V V} u\left(\mathbb{X}_{S}\right) d[X]_{s} .
$$

Regarding $I_{23}(\varepsilon, t)$, for every $\omega \in \Omega$, define $\psi_{\omega}:[0, T] \times[0,1] \times[0,1] \rightarrow \mathbb{R}$ as

$$
\psi_{\omega}(s, \alpha, \varepsilon):=(1-\alpha) \partial_{a a}^{2} \tilde{u}\left(\mathbb{X}_{s \mid[-T, 0[}(\omega), X_{s}(\omega)+\alpha\left(X_{s+\varepsilon}(\omega)-X_{s}(\omega)\right)\right)
$$


for all $(s, \alpha, \varepsilon) \in[0, T] \times[0,1] \times[0,1]$. Notice that $\psi_{\omega}$ is uniformly continuous. Denote $\rho_{\psi}$ its continuity modulus, then

$$
\sup _{t \in[0, T]}\left|I_{23}(\varepsilon, t)\right| \leq \int_{0}^{T} \rho_{\psi_{\omega}}(\varepsilon) \frac{\left(X_{s+\varepsilon}-X_{s}\right)^{2}}{\varepsilon} d s .
$$

Since $X$ has finite quadratic variation, we deduce that $I_{23}(\varepsilon, t) \rightarrow 0$ ucp as $\varepsilon \rightarrow 0^{+}$. Finally, because of $I_{0}(\varepsilon, t), I_{1}(\varepsilon, t), I_{22}(\varepsilon, t)$, and $I_{23}(\varepsilon, t)$ converge ucp, it follows that the forward integral exists:

$$
I_{21}(\varepsilon, t) \underset{\varepsilon \rightarrow 0^{+}}{\stackrel{\mathrm{ucp}}{\longrightarrow}} \int_{0}^{t} \partial_{a} \tilde{u}\left(\mathbb{X}_{S \mid[-T, 0[}, X_{s}\right) d^{-} X_{s}=\int_{0}^{t} D^{V} u\left(\mathbb{X}_{S}\right) d^{-} X_{s},
$$

from which the claim follows.

Remark 6 Notice that, under the hypotheses of Theorem 2, the forward integral $\int_{0}^{t} D^{V} \mathscr{U}\left(\mathbb{X}_{s}\right) d^{-} X_{s}$ exists as a ucp limit, which is generally not required.

Remark 7 The definition of horizontal derivative. Notice that our definition of horizontal derivative differs from that introduced in [17], since it is based on a limit on the left, while the definition proposed in [17] would conduct to the formula

$$
D^{H,+} u(\eta):=\lim _{\varepsilon \rightarrow 0^{+}} \frac{\tilde{u}\left(\eta(\cdot+\varepsilon) 1_{[-T, 0[}, \eta(0)\right)-\tilde{u}\left(\eta(\cdot) 1_{[-T, 0[}, \eta(0)\right)}{\varepsilon} .
$$

To give an insight into the difference between (16) and (26), let us consider a real continuous finite quadratic variation process $X$ with associated window process $\mathbb{X}$. Then, in the definition (26) of $D^{H,+} u\left(\mathbb{X}_{t}\right)$ we consider the increment $\tilde{u}\left(\mathbb{X}_{t \mid[-T, 0[}(\cdot+\varepsilon), X_{t}\right)-\tilde{u}\left(\mathbb{X}_{t \mid[-T, 0[}, X_{t}\right)$, comparing the present value of $u\left(\mathbb{X}_{t}\right)=\tilde{u}\left(\mathbb{X}_{t \mid[-T, 0[}, X_{t}\right)$ with an hypothetical future value $\tilde{u}\left(\mathbb{X}_{t \mid[-T, 0[}(\cdot+\varepsilon), X_{t}\right)$, obtained assuming a constant time evolution for $X$. On the other hand, in our definition (16) we consider the increment $\tilde{u}\left(\mathbb{X}_{t \mid[-T, 0[}, X_{t}\right)-\tilde{u}\left(\mathbb{X}_{t-\varepsilon \mid[-T, 0[}, X_{t}\right)$, where only the present and past values of $X$ are taken into account, and where we also extend in a constant way the trajectory of $X$ before time 0 . In particular, unlike (26), since we do not call in the future in our formula (16), we do not have to specify a future time evolution for $X$, but only a past evolution before time 0 . This difference between (16) and (26) is crucial for the proof of the functional Itô's formula. In particular, the adoption of (26) as definition for the horizontal derivative would require an additional regularity condition on $u$ in order to prove an Itô formula for the process $t \mapsto u\left(\mathbb{X}_{t}\right)$. Indeed, as it can be seen from the proof of Theorem 2, to prove Itô's formula we are led to consider the term

$$
I_{1}(\varepsilon, t)=\int_{0}^{t} \frac{\tilde{u}\left(\mathbb{X}_{s+\varepsilon \mid[-T, 0[}, X_{s+\varepsilon}\right)-\tilde{u}\left(\mathbb{X}_{s \mid[-T, 0[}, X_{s+\varepsilon}\right)}{\varepsilon} d s .
$$


When adopting definition (26) it is convenient to write $I_{1}(\varepsilon, t)$ as the sum of the two integrals

$$
\begin{aligned}
& I_{11}(\varepsilon, t)=\int_{0}^{t} \frac{\tilde{u}\left(\mathbb{X}_{s+\varepsilon \mid[-T, 0[}, X_{s+\varepsilon}\right)-\tilde{u}\left(\mathbb{X}_{s \mid[-T, 0[}(\cdot+\varepsilon), X_{s+\varepsilon}\right)}{\varepsilon} d s \\
& I_{12}(\varepsilon, t)=\int_{0}^{t} \frac{\tilde{u}\left(\mathbb{X}_{s \mid[-T, 0[}(\cdot+\varepsilon), X_{s+\varepsilon}\right)-\tilde{u}\left(\mathbb{X}_{s \mid[-T, 0[}, X_{s+\varepsilon}\right)}{\varepsilon} d s .
\end{aligned}
$$

It can be shown quite easily that, under suitable regularity conditions on $u$ (more precisely, if $u$ is continuous, $D^{H,+} u$ exists everywhere on $\mathscr{C}([-T, 0])$, and for every $\gamma \in \mathscr{C}\left(\left[-T, 0[)\right.\right.$ the map $(\varepsilon, a) \longmapsto D^{H,+} \tilde{u}(\gamma(\cdot+\varepsilon), a)$ is continuous on $[0, \infty) \times$ $\mathbb{R}[$, we have

$$
I_{12}(\varepsilon, t) \underset{\varepsilon \rightarrow 0^{+}}{\stackrel{\mathrm{ucp}}{\longrightarrow}} \int_{0}^{t} D^{H,+} u\left(\mathbb{X}_{s}\right) d s
$$

To conclude the proof of Itô's formula along the same lines as in Theorem 2, we should prove

$$
I_{11}(\varepsilon, t) \underset{\varepsilon \rightarrow 0^{+}}{\stackrel{\mathrm{ucp}}{\longrightarrow}} 0
$$

In order to guarantee (27), we need to impose some additional regularity condition on $\tilde{u}$, and hence on $u$. As an example, (27) is satisfied if we assume the following condition on $\tilde{u}$ : there exists a constant $C>0$ such that, for every $\varepsilon>0$,

$$
\left|\tilde{u}\left(\gamma_{1}, a\right)-\tilde{u}\left(\gamma_{2}, a\right)\right| \leq C \varepsilon \sup _{x \in[-\varepsilon, 0[}\left|\gamma_{1}(x)-\gamma_{2}(x)\right|
$$

for all $\gamma_{1}, \gamma_{2} \in \mathscr{C}\left(\left[-T, 0[)\right.\right.$ and $a \in \mathbb{R}$, with $\gamma_{1}(x)=\gamma_{2}(x)$ for any $x \in[-T,-\varepsilon]$. This last condition is verified if, for example, $\tilde{u}$ is uniformly Lipschitz continuous with respect to the $L^{1}([-T, 0])$-norm on $\mathscr{C}([-T, 0[)$, namely: there exists a constant $C>0$ such that

$$
\left|\tilde{u}\left(\gamma_{1}, a\right)-\tilde{u}\left(\gamma_{2}, a\right)\right| \leq C \int_{[-T, 0[}\left|\gamma_{1}(x)-\gamma_{2}(x)\right| d x,
$$

for all $\gamma_{1}, \gamma_{2} \in \mathscr{C}([-T, 0[)$ and $a \in \mathbb{R}$.

We conclude this subsection providing the functional Itô's formula for a map $\mathscr{U}:[0, T] \times C([-T, 0]) \rightarrow \mathbb{R}$ depending also on the time variable. Firstly, we notice that for a map $\mathscr{U}:[0, T] \times C([-T, 0]) \rightarrow \mathbb{R}($ resp. $u:[0, T] \times \mathscr{C}([-T, 0]) \rightarrow \mathbb{R})$ the functional derivatives $D^{H} \mathscr{U}, D^{V} \mathscr{U}$, and $D^{V V} \mathscr{U}$ (resp. $D^{H} u, D^{V} u$, and $D^{V V} u$ ) are defined in an obvious way as in Definition 11 (resp. Definition 9). Moreover, given $u:[0, T] \times \mathscr{C}([-T, 0]) \rightarrow \mathbb{R}$ we can define, as in Definition 8, a map $\tilde{u}:[0, T] \times$ $\mathscr{C}([-T, 0[) \times \mathbb{R} \rightarrow \mathbb{R}$. Then, we can give the following definitions. 
Definition 13 Let $I$ be $[0, T$ [ or $[0, T]$. We say that $u: I \times \mathscr{C}([-T, 0]) \rightarrow \mathbb{R}$ is of class $\mathscr{C}^{1,2}((I \times$ past $) \times$ present $)$ if the properties below hold.

(i) $u$ is continuous;

(ii) $\partial_{t} u$ exists everywhere on $I \times \mathscr{C}([-T, 0])$ and is continuous;

(iii) $D^{H} u$ exists everywhere on $I \times \mathscr{C}([-T, 0])$ and for every $\gamma \in \mathscr{C}([-T, 0[)$ the map

$$
(t, \varepsilon, a) \longmapsto D^{H} \tilde{u}(t, \gamma(\cdot-\varepsilon), a), \quad(t, \varepsilon, a) \in I \times[0, \infty[\times \mathbb{R}
$$

is continuous on $I \times[0, \infty[\times \mathbb{R}$;

(iv) $D^{V} u$ and $D^{V V} u$ exist everywhere on $I \times \mathscr{C}([-T, 0])$ and are continuous.

Definition 14 Let $I$ be $[0, T$ or $[0, T]$. We say that $\mathscr{U}: I \times C([-T, 0]) \rightarrow \mathbb{R}$ is $C^{1,2}((I \times$ past $) \times$ present $)$ if $\mathscr{U}$ admits a (necessarily unique) extension $u: I \times$ $\mathscr{C}([-T, 0]) \rightarrow \mathbb{R}$ of class $\mathscr{C}^{1,2}((I \times$ past $) \times$ present $)$.

We can now state the functional Itô's formula, whose proof is not reported, since it can be done along the same lines as Theorem 2 .

Theorem 3 Let $\mathscr{U}:[0, T] \times C([-T, 0]) \rightarrow \mathbb{R}$ be of class $C^{1,2}(([0, T] \times$ past $) \times$ present) and $X=\left(X_{t}\right)_{t \in[0, T]}$ be a real continuous finite quadratic variation process. Then, the following functional Itô's formula holds, $\mathbb{P}$-a.s.,

$$
\begin{aligned}
\mathscr{U}\left(t, \mathbb{X}_{t}\right)= & \mathscr{U}\left(0, \mathbb{X}_{0}\right)+\int_{0}^{t}\left(\partial_{t} \mathscr{U}\left(s, \mathbb{X}_{s}\right)+D^{H} \mathscr{U}\left(s, \mathbb{X}_{s}\right)\right) d s+\int_{0}^{t} D^{V} \mathscr{U}\left(s, \mathbb{X}_{s}\right) d^{-} X_{s} \\
& +\frac{1}{2} \int_{0}^{t} D^{V V} \mathscr{U}\left(s, \mathbb{X}_{s}\right) d[X]_{s},
\end{aligned}
$$

for all $0 \leq t \leq T$.

Remark 8 Notice that, as a particular case, choosing $\mathscr{U}(t, \eta)=F(t, \eta(0))$, for any $(t, \eta) \in[0, T] \times C([-T, 0])$, with $F \in C^{1,2}([0, T] \times \mathbb{R})$, we retrieve the classical Itô formula for finite quadratic variation processes, i.e. (4). More precisely, in this case $\mathscr{U}$ admits as unique continuous extension the map $u:[0, T] \times \mathscr{C}([-T, 0]) \rightarrow \mathbb{R}$ given by $u(t, \eta)=F(t, \eta(0))$, for all $(t, \eta) \in[0, T] \times \mathscr{C}([-T, 0])$. Moreover, we see that $D^{H} \mathscr{U} \equiv 0$, while $D^{V} \mathscr{U}=\partial_{x} F$ and $D^{V V} \mathscr{U}=\partial_{x x}^{2} F$, where $\partial_{x} F$ (resp. $\partial_{x x}^{2} F$ ) denotes the first-order (resp. second-order) partial derivative of $F$ with respect to its second argument.

\subsection{Comparison with Banach Space Valued Calculus via Regularization}

In the present subsection our aim is to make a link between functional Itô calculus, as derived in this paper, and Banach space valued stochastic calculus via regularization 
for window processes, which has been conceived in [13], see also [12, 14-16] for more recent developments. More precisely, our purpose is to identify the building blocks of our functional Itô's formula (19) with the terms appearing in the Itô formula derived in Theorem 6.3 and Sect.7.2 in [12]. While it is expected that the vertical derivative $D^{V} \mathscr{U}$ can be identified with the term $D_{d x}^{\delta_{0}} \mathscr{U}$ of the Fréchet derivative, it is more difficult to guess to which terms the horizontal derivative $D^{H} \mathscr{U}$ corresponds. To clarify this latter point, in this subsection we derive two formulae which express $D^{H} \mathscr{U}$ in terms of Fréchet derivatives of $\mathscr{U}$.

Let us introduce some useful notations. We denote by $B V([-T, 0])$ the set of càdlàg bounded variation functions on $[-T, 0]$, which is a Banach space when equipped with the norm

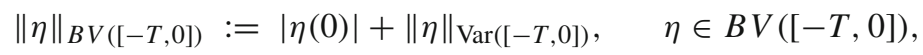

where $\|\eta\|_{\operatorname{Var}([-T, 0])}=|d \eta|([-T, 0])$ and $|d \eta|$ is the total variation measure associated to the measure $d \eta \in \mathscr{M}([-T, 0])$ generated by $\eta: d \eta(]-T,-t])=\eta(-t)-$ $\eta(-T), t \in[-T, 0]$. We recall from Sect. 2.1 that we extend $\eta \in B V([-T, 0])$ to all $x \in \mathbb{R}$ setting $\eta(x)=0, x<-T$, and $\eta(x)=\eta(0), x \geq 0$. Let us now introduce some useful facts about tensor products of Banach spaces.

Definition 15 Let $\left(E,\|\cdot\|_{E}\right)$ and $\left(F,\|\cdot\|_{F}\right)$ be two Banach spaces.

(i) We shall denote by $E \otimes F$ the algebraic tensor product of $E$ and $F$, defined as the set of elements of the form $v=\sum_{i=1}^{n} e_{i} \otimes f_{i}$, for some positive integer $n$, where $e \in E$ and $f \in F$. The map $\otimes: E \times F \rightarrow E \otimes F$ is bilinear.

(ii) We endow $E \otimes F$ with the projective norm $\pi$ :

$$
\pi(v):=\inf \left\{\sum_{i=1}^{n}\left\|e_{i}\right\|_{E}\left\|f_{i}\right\|_{F}: v=\sum_{i=1}^{n} e_{i} \otimes f_{i}\right\}, \quad \forall v \in E \otimes F
$$

(iii) We denote by $E \hat{\otimes}_{\pi} F$ the Banach space obtained as the completion of $E \otimes F$ for the norm $\pi$. We shall refer to $E \hat{\otimes}_{\pi} F$ as the tensor product of the Banach spaces $E$ and $F$.

(iv) If $E$ and $F$ are Hilbert spaces, we denote $E \hat{\otimes}_{h} F$ the Hilbert tensor product, which is still a Hilbert space obtained as the completion of $E \otimes F$ for the scalar product $\left\langle e^{\prime} \otimes f^{\prime}, e^{\prime \prime} \otimes f^{\prime \prime}\right\rangle:=\left\langle e^{\prime}, e^{\prime \prime}\right\rangle_{E}\left\langle f^{\prime}, f^{\prime \prime}\right\rangle_{F}$, for any $e^{\prime}, e^{\prime \prime} \in E$ and $f^{\prime}, f^{\prime \prime} \in F$.

(v) The symbols $E \hat{\otimes}_{\pi}^{2}$ and $e \otimes^{2}$ denote, respectively, the Banach space $E \hat{\otimes}_{\pi} E$ and the element $e \otimes e$ of the algebraic tensor product $E \otimes E$.

Remark 9 (i) The projective norm $\pi$ belongs to the class of the so-called reasonable crossnorms $\alpha$ on $E \otimes F$, verifying $\alpha(e \otimes f)=\|e\|_{E}\|f\|_{F}$.

(ii) We notice, proceeding for example as in [16] (see, in particular, formula (2.1) in [16]; for more information on this subject we refer to [41]), that the dual $\left(E \hat{\otimes}_{\pi} F\right)^{*}$ of 
$E \hat{\otimes}_{\pi} F$ is isomorphic to the space of continuous bilinear forms $\mathscr{B} i(E, F)$, equipped with the norm $\|\cdot\|_{E, F}$ defined as

$$
\|\Phi\|_{E, F}:=\sup _{\substack{e \in E, f \in F \\\|e\|_{E},\|f\|_{F} \leq 1}}|\Phi(e, f)|, \quad \forall \Phi \in \mathscr{B} i(E, F) .
$$

Moreover, there exists a canonical isomorphism between $\mathscr{B} i(E, F)$ and $L\left(E, F^{*}\right)$, the space of bounded linear operators from $E$ into $F^{*}$. Hence, we have the following chain of canonical identifications: $\left(E \hat{\otimes}_{\pi} F\right)^{*} \cong \mathscr{B} i(E, F) \cong L\left(E ; F^{*}\right)$.

Definition 16 Let $E$ be a Banach space. We say that $\mathscr{U}: E \rightarrow \mathbb{R}$ is of $\operatorname{class} C^{2}(E)$ if

(i) $D \mathscr{U}$, the first Fréchet derivative of $\mathscr{U}$, belongs to $C\left(E ; E^{*}\right)$ and

(ii) $D^{2} \mathscr{U}$, the second Fréchet derivative of $\mathscr{U}$, belongs to $C\left(E ; L\left(E ; E^{*}\right)\right)$.

Remark 10 Take $E=C([-T, 0])$ in Definition 16.

(i) First Fréchet derivative $D \mathscr{U}$. We have

$$
D \mathscr{U}: C([-T, 0]) \longrightarrow(C([-T, 0]))^{*} \cong \mathscr{M}([-T, 0])
$$

For every $\eta \in C([-T, 0])$, we shall denote $D_{d x} \mathscr{U}(\eta)$ the unique measure in $\mathscr{M}([-T, 0])$ such that

$$
D \mathscr{U}(\eta) \varphi=\int_{[-T, 0]} \varphi(x) D_{d x} \mathscr{U}(\eta), \quad \forall \varphi \in C([-T, 0]) .
$$

Notice that $\mathscr{M}([-T, 0])$ can be represented as the direct sum: $\mathscr{M}([-T, 0])=$ $\mathscr{M}_{0}([-T, 0]) \oplus \mathscr{D}_{0}$, where we recall that $\mathscr{M}_{0}([-T, 0])$ is the subset of $\mathscr{M}([-T, 0])$ of measures $\mu$ such that $\mu(\{0\})=0$, instead $\mathscr{D}_{0}$ (which is a shorthand for $\mathscr{D}_{0}([-T, 0])$ ) denotes the one-dimensional space of measures which are multiples of the Dirac measure $\delta_{0}$. For every $\eta \in C([-T, 0])$ we denote by $\left(D_{d x}^{\perp} \mathscr{U}(\eta), D_{d x}^{\delta_{0}} \mathscr{U}(\eta)\right)$ the unique pair in $\mathscr{M}_{0}([-T, 0]) \oplus \mathscr{D}_{0}$ such that

$$
D_{d x} \mathscr{U}(\eta)=D_{d x}^{\perp} \mathscr{U}(\eta)+D_{d x}^{\delta_{0}} \mathscr{U}(\eta)
$$

(ii) Second Fréchet derivative $D^{2} \mathscr{U}$. We have

$$
\begin{aligned}
D^{2} \mathscr{U}: C([-T, 0]) \longrightarrow L\left(C([-T, 0]) ;(C([-T, 0]))^{*}\right) & \cong \mathscr{B} i(C([-T, 0]), C([-T, 0])) \\
& \cong\left(C([-T, 0]) \hat{\otimes}_{\pi} C([-T, 0])\right)^{*}
\end{aligned}
$$

where we used the identifications of Remark 9(ii). Let $\eta \in C([-T, 0])$; a typical situation arises when there exists $D_{d x d y} \mathscr{U}(\eta) \in \mathscr{M}\left([-T, 0]^{2}\right)$ such that $D^{2} \mathscr{U}(\eta) \in$ $L\left(C([-T, 0]) ;(C([-T, 0]))^{*}\right)$ admits the representation 


$$
D^{2} \mathscr{U}(\eta)(\varphi, \psi)=\int_{[-T, 0]^{2}} \varphi(x) \psi(y) D_{d x d y} \mathscr{U}(\eta), \quad \forall \varphi, \psi \in C([-T, 0]) .
$$

Moreover, $D_{d x d y} \mathscr{U}(\eta)$ is uniquely determined.

The definition below was given in [13].

Definition 17 Let $E$ be a Banach space. A Banach subspace $\left(\chi,\|\cdot\|_{\chi}\right)$ continuously injected into $\left(E \hat{\otimes}_{\pi}^{2}\right)^{*}$, i.e., $\|\cdot\|_{\chi} \geq\|\cdot\|_{\left(E \hat{\otimes}_{\pi}^{2}\right)^{*}}$, will be called a Chi-subspace (of $\left.\left(E \hat{\otimes}_{\pi}^{2}\right)^{*}\right)$.

Remark 11 Take $E=C([-T, 0])$ in Definition 17. As indicated in [13], a typical example of Chi-subspace of $C([-T, 0]) \hat{\otimes}_{\pi}^{2}$ is $\mathscr{M}\left([-T, 0]^{2}\right)$ equipped with the usual total variation norm, denoted by $\|\cdot\|_{\text {Var }}$. Another important Chi-subspace of $C([-T, 0]) \hat{\otimes}_{\pi}^{2}$ is the following, which is also a Chi-subspace of $\mathscr{M}\left([-T, 0]^{2}\right)$ :

$$
\begin{aligned}
\chi_{0}:= & \left\{\mu \in \mathscr{M}\left([-T, 0]^{2}\right): \mu(d x, d y)=g_{1}(x, y) d x d y+\lambda_{1} \delta_{0}(d x) \otimes \delta_{0}(d y)\right. \\
& +g_{2}(x) d x \otimes \lambda_{2} \delta_{0}(d y)+\lambda_{3} \delta_{0}(d x) \otimes g_{3}(y) d y+g_{4}(x) \delta_{y}(d x) \otimes d y, \\
& \left.g_{1} \in L^{2}\left([-T, 0]^{2}\right), g_{2}, g_{3} \in L^{2}([-T, 0]), g_{4} \in L^{\infty}([-T, 0]), \lambda_{1}, \lambda_{2}, \lambda_{3} \in \mathbb{R}\right\} .
\end{aligned}
$$

Using the notations of Example 3.4 and Remark 3.5 in [16], to which we refer for more details on this subject, we notice that $\chi_{0}$ is indeed given by the direct sum $\chi_{0}=$ $L^{2}\left([-T, 0]^{2}\right) \oplus\left(L^{2}([-T, 0]) \hat{\otimes}_{h} \mathscr{D}_{0}\right) \oplus\left(\mathscr{D}_{0} \hat{\otimes}_{h} L^{2}([-T, 0])\right) \oplus \mathscr{D}_{0,0}\left([-T, 0]^{2}\right) \oplus$ $\operatorname{Diag}\left([-T, 0]^{2}\right)$. In the sequel, we shall refer to the term $g_{4}(x) \delta_{y}(d x) \otimes d y$ as the diagonal component and to $g_{4}(x)$ as the diagonal element of $\mu$.

We can now state our first representation result for $D^{H} \mathscr{U}$.

Proposition 7 Let $\mathscr{U}: C([-T, 0]) \rightarrow \mathbb{R}$ be continuously Fréchet differentiable. Suppose the following.

(i) For any $\eta \in C([-T, 0])$ there exists $D^{a c} \mathscr{U}(\eta) \in B V([-T, 0])$ such that

$$
D_{d x}^{\perp} \mathscr{U}(\eta)=D_{x}^{a c} \mathscr{U}(\eta) d x .
$$

(ii) There exist continuous extensions (necessarily unique)

$$
u: \mathscr{C}([-T, 0]) \rightarrow \mathbb{R}, \quad D^{a c} u: \mathscr{C}([-T, 0]) \rightarrow B V([-T, 0])
$$

of $\mathscr{U}$ and $D^{a c} \mathscr{U}$, respectively.

Then, for any $\eta \in C([-T, 0])$,

$$
D^{H} \mathscr{U}(\eta)=\int_{[-T, 0]} D_{x}^{a c} \mathscr{U}(\eta) d^{+} \eta(x)
$$


where we recall that the previous deterministic integral has been defined in Sect. 2.1.1. In particular, the horizontal derivative $D^{H} \mathscr{U}(\eta)$ and the backward integral in (29) exist.

Proof Let $\eta \in C([-T, 0])$, then starting from the left-hand side of (29), using the definition of $D^{H} \mathscr{U}(\eta)$, we are led to consider the following increment for the function $u$ :

$$
\frac{u(\eta)-u\left(\eta(\cdot-\varepsilon) 1_{[-T, 0[}+\eta(0) 1_{\{0\}}\right)}{\varepsilon} .
$$

We shall expand (30) using a Taylor's formula. Firstly, notice that, since $\mathscr{U}$ is $C^{1}$ Fréchet on $C([-T, 0])$, for every $\eta_{1} \in C([-T, 0])$, with $\eta_{1}(0)=\eta(0)$, from the fundamental theorem of calculus we have

$$
\mathscr{U}(\eta)-\mathscr{U}\left(\eta_{1}\right)=\int_{0}^{1}\left(\int_{-T}^{0} D_{x}^{\mathrm{ac}} \mathscr{U}\left(\eta+\lambda\left(\eta_{1}-\eta\right)\right)\left(\eta(x)-\eta_{1}(x)\right) d x\right) d \lambda .
$$

Recalling from Remark 3 the density of $C_{\eta(0)}([-T, 0])$ in $\mathscr{C}_{\eta(0)}([-T, 0])$ with respect to the topology of $\mathscr{C}([-T, 0])$, we deduce the following Taylor's formula for $u$ :

$$
u(\eta)-u\left(\eta_{1}\right)=\int_{0}^{1}\left(\int_{-T}^{0} D_{x}^{\mathrm{ac}} u\left(\eta+\lambda\left(\eta_{1}-\eta\right)\right)\left(\eta(x)-\eta_{1}(x)\right) d x\right) d \lambda,
$$

for all $\eta_{1} \in \mathscr{C}_{\eta(0)}([-T, 0])$. As a matter of fact, for any $\left.\left.\delta \in\right] 0, T / 2\right]$ let (similarly to Remark 3(i))

$$
\eta_{1, \delta}(x):= \begin{cases}\eta_{1}(x), & -T \leq x \leq-\delta, \\ \frac{1}{\delta}\left(\eta_{1}(0)-\eta_{1}(-\delta)\right) x+\eta_{1}(0), & -\delta<x \leq 0\end{cases}
$$

and $\eta_{1,0}:=\eta_{1}$. Then $\eta_{1, \delta} \in C([-T, 0])$, for any $\left.\left.\delta \in\right] 0, T / 2\right]$, and $\eta_{1, \delta} \rightarrow \eta_{1}$ in $\mathscr{C}([-T, 0])$, as $\delta \rightarrow 0^{+}$. Now, define $f:[-T, 0] \times[0,1] \times[0, T / 2] \rightarrow \mathbb{R}$ as follows

$$
f(x, \lambda, \delta):=D_{x}^{\mathrm{ac}} u\left(\eta+\lambda\left(\eta_{1, \delta}-\eta\right)\right)\left(\eta(x)-\eta_{1, \delta}(x)\right),
$$

for all $(x, \lambda, \delta) \in[-T, 0] \times[0,1] \times[0, T / 2]$. Now $(\lambda, \delta) \mapsto \eta+\lambda\left(\eta_{1, \delta}-\eta\right)$, is continuous. Taking into account that $D^{\mathrm{ac}} u: \mathscr{C}([-T, 0]) \rightarrow B V([-T, 0])$ is continuous, hence bounded on compact sets, it follows that $f$ is bounded. Then, it follows from Lebesgue dominated convergence theorem that 


$$
\begin{aligned}
& \int_{0}^{1}\left(\int_{-T}^{0} D_{x}^{\mathrm{ac}} \mathscr{U}\left(\eta+\lambda\left(\eta_{1, \delta}-\eta\right)\right)\left(\eta(x)-\eta_{1, \delta}(x)\right) d x\right) d \lambda \\
& =\int_{0}^{1}\left(\int_{-T}^{0} f(x, \lambda, \delta) d x\right) d \lambda \stackrel{\delta \rightarrow 0^{+}}{\longrightarrow} \int_{0}^{1}\left(\int_{-T}^{0} f(x, \lambda, 0) d x\right) d \lambda \\
& =\int_{0}^{1}\left(\int_{-T}^{0} D_{x}^{\mathrm{ac}} u\left(\eta+\lambda\left(\eta_{1}-\eta\right)\right)\left(\eta(x)-\eta_{1}(x)\right) d x\right) d \lambda,
\end{aligned}
$$

from which we deduce $(31)$, since $\mathscr{U}\left(\eta_{1, \delta}\right) \rightarrow u\left(\eta_{1}\right)$ as $\delta \rightarrow 0^{+}$. Taking $\eta_{1}(\cdot)=$ $\eta(\cdot-\varepsilon) 1_{[-T, 0[}+\eta(0) 1_{\{0\}}$, we obtain

$$
\begin{aligned}
& \frac{u(\eta)-u\left(\eta(\cdot-\varepsilon) 1_{[-T, 0[}+\eta(0) 1_{\{0\}}\right)}{\varepsilon} \\
& =\int_{0}^{1}\left(\int_{-T}^{0} D_{x}^{\mathrm{ac}} u\left(\eta+\lambda(\eta(\cdot-\varepsilon)-\eta(\cdot)) 1_{[-T, 0[}\right) \frac{\eta(x)-\eta(x-\varepsilon)}{\varepsilon} d x\right) d \lambda \\
& =I_{1}(\eta, \varepsilon)+I_{2}(\eta, \varepsilon)+I_{3}(\eta, \varepsilon),
\end{aligned}
$$

where

$$
\begin{aligned}
I_{1}(\eta, \varepsilon):= & \int_{0}^{1}\left(\int _ { - T } ^ { 0 } \eta ( x ) \frac { 1 } { \varepsilon } \left(D_{x}^{\mathrm{ac}} u\left(\eta+\lambda(\eta(\cdot-\varepsilon)-\eta(\cdot)) 1_{[-T, 0[}\right)\right.\right. \\
& \left.\left.-D_{x+\varepsilon}^{\mathrm{ac}} u\left(\eta+\lambda(\eta(\cdot-\varepsilon)-\eta(\cdot)) 1_{[-T, 0[}\right)\right) d x\right) d \lambda \\
I_{2}(\eta, \varepsilon):= & \frac{1}{\varepsilon} \int_{0}^{1}\left(\int_{-\varepsilon}^{0} \eta(x) D_{x+\varepsilon}^{\mathrm{ac}} u\left(\eta+\lambda(\eta(\cdot-\varepsilon)-\eta(\cdot)) 1_{[-T, 0[}\right) d x\right) d \lambda, \\
I_{3}(\eta, \varepsilon):= & -\frac{1}{\varepsilon} \int_{0}^{1}\left(\int_{-T-\varepsilon}^{-T} \eta(x) D_{x+\varepsilon}^{\mathrm{ac}} u\left(\eta+\lambda(\eta(\cdot-\varepsilon)-\eta(\cdot)) 1_{[-T, 0[}\right) d x\right) d \lambda .
\end{aligned}
$$

Notice that, since $\eta(x)=0$ for $x<-T$, we see that $I_{3}(\eta, \varepsilon)=0$. Moreover $D_{x}^{\mathrm{ac}} u(\cdot)=D_{0}^{\mathrm{ac}} u(\cdot)$, for $x \geq 0$, and $\eta+\lambda(\eta(\cdot-\varepsilon)-\eta(\cdot)) 1_{[-T, 0[} \rightarrow \eta$ in $\mathscr{C}([-T, 0])$ as $\varepsilon \rightarrow 0^{+}$. Since $D_{x}^{\text {ac }} u$ is continuous from $\mathscr{C}([-T, 0])$ into $B V([-T, 0])$, we have $D_{0}^{\mathrm{ac}} u\left(\eta+\lambda(\eta(\cdot-\varepsilon)-\eta(\cdot)) 1_{[-T, 0[}\right) \rightarrow D_{0}^{\mathrm{ac}} u(\eta)$ as $\varepsilon \rightarrow 0^{+}$. Then

$$
\begin{aligned}
& \frac{1}{\varepsilon} \int_{-\varepsilon}^{0} \eta(x) D_{x+\varepsilon}^{\mathrm{ac}} u\left(\eta+\lambda(\eta(\cdot-\varepsilon)-\eta(\cdot)) 1_{[-T, 0[}\right) d x \\
& =\frac{1}{\varepsilon} \int_{-\varepsilon}^{0} \eta(x) d x D_{0}^{\mathrm{ac}} u\left(\eta+\lambda(\eta(\cdot-\varepsilon)-\eta(\cdot)) 1_{[-T, 0[} \stackrel{\varepsilon \rightarrow 0^{+}}{\longrightarrow} \eta(0) D_{0}^{\mathrm{ac}} u(\eta) .\right.
\end{aligned}
$$

So $I_{2}(\eta, \varepsilon) \rightarrow \eta(0) D_{0}^{\mathrm{ac}} u(\eta)$. Finally, concerning $I_{1}(\eta, \varepsilon)$, from Fubini's theorem we obtain (denoting $\left.\eta_{\varepsilon, \lambda}:=\eta+\lambda(\eta(\cdot-\varepsilon)-\eta(\cdot)) 1_{[-T, 0[}\right)$ 


$$
\begin{aligned}
I_{1}(\eta, \varepsilon) & =\int_{0}^{1}\left(\int_{-T}^{0} \eta(x) \frac{1}{\varepsilon}\left(D_{x}^{\mathrm{ac}} u\left(\eta_{\varepsilon, \lambda}\right)-D_{x+\varepsilon}^{\mathrm{ac}} u\left(\eta_{\varepsilon, \lambda}\right)\right) d x\right) d \lambda \\
& =-\int_{0}^{1}\left(\int_{-T}^{0} \eta(x) \frac{1}{\varepsilon}\left(\int_{] x, x+\varepsilon]} D_{d y}^{\mathrm{ac}} u\left(\eta_{\varepsilon, \lambda}\right)\right) d x\right) d \lambda \\
& =-\int_{0}^{1}\left(\int_{]-T, \varepsilon]} \frac{1}{\varepsilon}\left(\int_{(-T) \vee(y-\varepsilon)}^{0 \wedge y} \eta(x) d x\right) D_{d y}^{\mathrm{ac}} u\left(\eta_{\varepsilon, \lambda}\right)\right) d \lambda \\
& =I_{11}(\eta, \varepsilon)+I_{12}(\eta, \varepsilon),
\end{aligned}
$$

where

$$
\begin{aligned}
I_{11}(\eta, \varepsilon) & :=-\int_{0}^{1}\left(\int_{]-T, \varepsilon]} \frac{1}{\varepsilon}\left(\int_{(-T) \vee(y-\varepsilon)}^{0 \wedge y} \eta(x) d x\right)\left(D_{d y}^{\mathrm{ac}} u\left(\eta_{\varepsilon, \lambda}\right)-D_{d y}^{\mathrm{ac}} u(\eta)\right)\right) d \lambda, \\
I_{12}(\eta, \varepsilon) & :=-\int_{0}^{1}\left(\int_{]-T, \varepsilon]} \frac{1}{\varepsilon}\left(\int_{(-T) \vee(y-\varepsilon)}^{0 \wedge y} \eta(x) d x\right) D_{d y}^{\mathrm{ac}} u(\eta)\right) d \lambda \\
& =-\left(\int_{]-T, \varepsilon]} \frac{1}{\varepsilon}\left(\int_{(-T) \vee(y-\varepsilon)}^{0 \wedge y} \eta(x) d x\right) D_{d y}^{\mathrm{ac}} u(\eta) .\right.
\end{aligned}
$$

Recalling that $D_{x}^{\mathrm{ac}} u(\cdot)=D_{0}^{\mathrm{ac}} u(\cdot)$, for $x \geq 0$, we see that in $I_{11}(\eta, \varepsilon)$ and $I_{12}(\eta, \varepsilon)$ the integrals on $]-T, \varepsilon]$ are equal to the same integrals on $]-T, 0]$, i.e.,

$$
\begin{aligned}
I_{11}(\eta, \varepsilon) & =-\int_{0}^{1}\left(\int_{]-T, 0]} \frac{1}{\varepsilon}\left(\int_{(-T) \vee(y-\varepsilon)}^{0 \wedge y} \eta(x) d x\right)\left(D_{d y}^{\mathrm{ac}} u\left(\eta_{\varepsilon, \lambda}\right)-D_{d y}^{\mathrm{ac}} u(\eta)\right)\right) d \lambda \\
& =-\int_{0}^{1}\left(\int_{]-T, 0]} \frac{1}{\varepsilon}\left(\int_{y-\varepsilon}^{y} \eta(x) d x\right)\left(D_{d y}^{\mathrm{ac}} u\left(\eta_{\varepsilon, \lambda}\right)-D_{d y}^{\mathrm{ac}} u(\eta)\right)\right) d \lambda \\
I_{12}(\eta, \varepsilon) & =-\int_{]-T, 0]} \frac{1}{\varepsilon}\left(\int_{(-T) \vee(y-\varepsilon)}^{0 \wedge y} \eta(x) d x\right) D_{d y}^{\mathrm{ac}} u(\eta) \\
& =-\int_{]-T, 0]} \frac{1}{\varepsilon}\left(\int_{y-\varepsilon}^{y} \eta(x) d x\right) D_{d y}^{\mathrm{ac}} u(\eta) .
\end{aligned}
$$

Now, observe that

$$
\left|I_{11}(\eta, \varepsilon)\right| \leq\|\eta\|_{\infty}\left\|D_{\cdot}^{\mathrm{ac}} u\left(\eta_{\varepsilon, \lambda}\right)-D_{\cdot}^{\mathrm{ac}} u(\eta)\right\|_{\operatorname{Var}([-T, 0])} \stackrel{\varepsilon \rightarrow 0^{+}}{\longrightarrow} 0 .
$$

Moreover, since $\eta$ is continuous at $y \in]-T, 0]$, we deduce that $\int_{y-\varepsilon}^{y} \eta(x) d x / \varepsilon \rightarrow$ $\eta(y)$ as $\varepsilon \rightarrow 0^{+}$. Therefore, by Lebesgue's dominated convergence theorem, we get

$$
I_{12}(\eta, \varepsilon) \stackrel{\varepsilon \rightarrow 0^{+}}{\longrightarrow}-\int_{]-T, 0]} \eta(y) D_{d y}^{\mathrm{ac}} u(\eta) .
$$


So $I_{1}(\eta, \varepsilon) \rightarrow-\int_{]-T, 0]} \eta(y) D_{d y}^{\mathrm{ac}} u(\eta)$. In conclusion, we have

$$
D^{H} \mathscr{U}(\eta)=\eta(0) D_{0}^{\mathrm{ac}} u(\eta)-\int_{]-T, 0]} \eta(y) D_{d y}^{\mathrm{ac}} u(\eta) .
$$

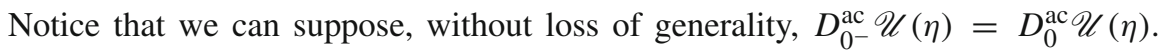
Then, the above identity gives (29) using the integration by parts formula (12).

For our second representation result of $D^{H} \mathscr{U}$ we need the following generalization of the deterministic backward integral when the integrand is a measure.

Definition 18 Let $a<b$ be two reals. Let $f:[a, b] \rightarrow \mathbb{R}$ be a càdlàg function (resp. càdlàg function with $\mathrm{f}(\mathrm{a})=0)$ and $g \in \mathscr{M}([-T, 0])$. Suppose that the following limit

$$
\begin{aligned}
\int_{[a, b]} g(d s) d^{+} f(s) & :=\lim _{\varepsilon \rightarrow 0^{+}} \int_{[a, b]} g(d s) \frac{f_{\bar{J}}(s)-f_{\bar{J}}(s-\varepsilon)}{\varepsilon} \\
\text { (resp. } \int_{[a, b]} g(d s) d^{-} f(s) & \left.:=\lim _{\varepsilon \rightarrow 0^{+}} \int_{[a, b]} g(d s) \frac{f_{\bar{J}}(s+\varepsilon)-f_{\bar{J}}(s)}{\varepsilon}\right)
\end{aligned}
$$

exists and it is finite. Then, the obtained quantity is denoted by $\int_{[a, b]} g d^{+} f$ $\left(\int_{[a, b]} g d^{-} f\right)$ and called (deterministic, definite) backward (resp. forward) integral of $g$ with respect to $f($ on $[a, b])$.

Proposition 8 If $g$ is absolutely continuous with density being càdlàg (still denoted with $g$ ) then Definition 18 is compatible with the one in Definition 4.

Proof Suppose that $g(d s)=g(s) d s$ with $g$ càdlàg.

Identity (32). The right-hand side of (6) gives

$$
\int_{a}^{b} g(s) \frac{f(s)-f(s-\varepsilon)}{\varepsilon} d s,
$$

which is also the right-hand side of (32) in that case.

Identity (33). The right-hand side of (5) gives, since $f(a)=0$,

$$
\frac{1}{\varepsilon} g(a) \int_{a-\varepsilon}^{a} f(s+\varepsilon) d s+\int_{a}^{b} g(s) \frac{f_{\bar{J}}(s+\varepsilon)-f_{\bar{J}}(s)}{\varepsilon} d s
$$

The first integral goes to zero. The second one equals the right-hand side of (33). 
Proposition 9 Let $\eta \in C([-T, 0])$ be such that the quadratic variation on $[-T, 0]$ exists. Let $\mathscr{U}: C([-T, 0]) \rightarrow \mathbb{R}$ be twice continuously Fréchet differentiable such that

$D^{2} \mathscr{U}: C([-T, 0]) \longrightarrow \chi_{0} \subset\left(C([-T, 0]) \hat{\otimes}_{\pi} C([-T, 0])\right)^{*}$ continuously with respect to $\chi_{0}$.

Let us also suppose the following.

(i) $D_{x}^{2, \text { Diag }} \mathscr{U}(\eta)$, the diagonal element of the second-order derivative at $\eta$, has a set of discontinuity which has null measure with respect to $[\eta]$ (in particular, if it is countable).

(ii) There exist continuous extensions (necessarily unique):

$$
u: \mathscr{C}([-T, 0]) \rightarrow \mathbb{R}, \quad D_{d x d y}^{2} u: \mathscr{C}([-T, 0]) \rightarrow \chi_{0}
$$

of $\mathscr{U}$ and $D_{d x d y}^{2} \mathscr{U}$, respectively.

(iii) The horizontal derivative $D^{H} \mathscr{U}(\eta)$ exists at $\eta \in C([-T, 0])$.

Then

$$
D^{H} \mathscr{U}(\eta)=\int_{[-T, 0]} D_{d x}^{\perp} \mathscr{U}(\eta) d^{+} \eta(x)-\frac{1}{2} \int_{[-T, 0]} D_{x}^{2, \operatorname{Diag} \mathscr{U}(\eta) d[\eta](x) .}
$$

In particular, the backward integral in (34) exists.

Proof Let $\eta \in C([-T, 0])$. Using the definition of $D^{H} \mathscr{U}(\eta)$ we are led to consider the following increment for the function $u$ :

$$
\frac{u(\eta)-u\left(\eta(\cdot-\varepsilon) 1_{[-T, 0[}+\eta(0) 1_{\{0\}}\right)}{\varepsilon},
$$

with $\varepsilon>0$. Our aim is to expand (35) using a Taylor's formula. To this end, since $\mathscr{U}$ is $C^{2}$ Fréchet, we begin noting that for every $\eta_{1} \in C([-T, 0])$ the following standard Taylor's expansion holds:

$$
\begin{aligned}
\mathscr{U}\left(\eta_{1}\right)= & \mathscr{U}(\eta)+\int_{[-T, 0]} D_{d x} \mathscr{U}(\eta)\left(\eta_{1}(x)-\eta(x)\right) \\
& +\frac{1}{2} \int_{[-T, 0]^{2}} D_{d x d y}^{2} \mathscr{U}(\eta)\left(\eta_{1}(x)-\eta(x)\right)\left(\eta_{1}(y)-\eta(y)\right) \\
& +\int_{0}^{1}(1-\lambda)\left(\int _ { [ - T , 0 ] ^ { 2 } } \left(D_{d x d y}^{2} \mathscr{U}\left(\eta+\lambda\left(\eta_{1}-\eta\right)\right)\right.\right. \\
& \left.\left.-D_{d x d y}^{2} \mathscr{U}(\eta)\right)\left(\eta_{1}(x)-\eta(x)\right)\left(\eta_{1}(y)-\eta(y)\right)\right) d \lambda
\end{aligned}
$$


Now, using the density of $C_{\eta(0)}([-T, 0])$ into $\mathscr{C}_{\eta(0)}([-T, 0])$ with respect to the topology of $\mathscr{C}([-T, 0])$ and proceeding as in the proof of Proposition 7 , we deduce the following Taylor's formula for $u$ :

$$
\begin{aligned}
& u(\eta)-u\left(\eta(\cdot-\varepsilon) 1_{[-T, 0[}+\eta(0) 1_{\{0\}}\right) \\
= & \int_{[-T, 0]} D_{d x}^{\perp} \mathscr{U}(\eta) \frac{\eta(x)-\eta(x-\varepsilon)}{\varepsilon} \\
& -\frac{1}{2} \int_{[-T, 0]^{2}} D_{d x d y}^{2} \mathscr{U}(\eta) \frac{(\eta(x)-\eta(x-\varepsilon))(\eta(y)-\eta(y-\varepsilon))}{\varepsilon} 1_{[-T, 0[\times[-T, 0[}(x, y) \\
& -\int_{0}^{1}(1-\lambda)\left(\int _ { [ - T , 0 ] ^ { 2 } } \left(D_{d x d y}^{2} u\left(\eta+\lambda(\eta(\cdot-\varepsilon)-\eta(\cdot)) 1_{[-T, 0[}\right)\right.\right. \\
& \left.\left.-D_{d x d y}^{2} \mathscr{U}(\eta)\right) \frac{(\eta(x)-\eta(x-\varepsilon))(\eta(y)-\eta(y-\varepsilon))}{\varepsilon} 1_{[-T, 0[\times[-T, 0[}(x, y)\right) d \lambda .
\end{aligned}
$$

Recalling the definition of $\chi_{0}$ given in Remark 11, we notice that (due to the presence of the indicator function $\left.1_{[-T, 0[\times[-T, 0[}\right)$

$$
\begin{aligned}
& \int_{[-T, 0]^{2}} D_{d x d y}^{2} \mathscr{U}(\eta) \frac{(\eta(x)-\eta(x-\varepsilon))(\eta(y)-\eta(y-\varepsilon))}{\varepsilon} 1_{[-T, 0[\times[-T, 0[}(x, y) \\
& =\int_{[-T, 0]^{2}} D_{x y}^{2, L^{2}} \mathscr{U}(\eta) \frac{(\eta(x)-\eta(x-\varepsilon))(\eta(y)-\eta(y-\varepsilon))}{\varepsilon} d x d y \\
& +\int_{[-T, 0]} D_{x}^{2, \operatorname{Diag}} \mathscr{U}(\eta) \frac{(\eta(x)-\eta(x-\varepsilon))^{2}}{\varepsilon} d x,
\end{aligned}
$$

where, by hypothesis, the maps $\eta \in \mathscr{C}([-T, 0]) \mapsto D_{x}^{2, L^{2}} u(\eta) \in L^{2}\left([-T, 0]^{2}\right)$ and $\eta \in \mathscr{C}([-T, 0]) \mapsto D_{x}^{2, \operatorname{Diag}} u(\eta) \in L^{\infty}([-T, 0])$ are continuous. In particular, (36) becomes

$$
\frac{u(\eta)-u\left(\eta(\cdot-\varepsilon) 1_{[-T, 0[}+\eta(0) 1_{\{0\}}\right)}{\varepsilon}=I_{1}(\varepsilon)+I_{2}(\varepsilon)+I_{3}(\varepsilon)+I_{4}(\varepsilon)+I_{5}(\varepsilon),
$$

where

$$
\begin{aligned}
& I_{1}(\varepsilon):=\int_{[-T, 0]} D_{d x}^{\perp} \mathscr{U}(\eta) \frac{\eta(x)-\eta(x-\varepsilon)}{\varepsilon}, \\
& I_{2}(\varepsilon):=-\frac{1}{2} \int_{[-T, 0]^{2}} D_{x y}^{2, L^{2}} \mathscr{U}(\eta) \frac{(\eta(x)-\eta(x-\varepsilon))(\eta(y)-\eta(y-\varepsilon))}{\varepsilon} d x d y, \\
& I_{3}(\varepsilon):=-\frac{1}{2} \int_{[-T, 0]} D_{x}^{2, \operatorname{Diag}} \mathscr{U}(\eta) \frac{(\eta(x)-\eta(x-\varepsilon))^{2}}{\varepsilon} d x,
\end{aligned}
$$




$$
\begin{aligned}
& I_{4}(\varepsilon):=-\int_{0}^{1}(1-\lambda)\left(\int _ { [ - T , 0 ] ^ { 2 } } \left(D_{x y}^{2, L^{2}} u\left(\eta+\lambda(\eta(\cdot-\varepsilon)-\eta(\cdot)) 1_{[-T, 0[}\right)\right.\right. \\
& \left.\left.-D_{x y}^{2, L^{2}} \mathscr{U}(\eta)\right) \frac{(\eta(x)-\eta(x-\varepsilon))(\eta(y)-\eta(y-\varepsilon))}{\varepsilon} d x d y\right) d \lambda, \\
& I_{5}(\varepsilon):=-\int_{0}^{1}(1-\lambda)\left(\int _ { [ - T , 0 ] } \left(D_{x}^{2, \text { Diag }} u\left(\eta+\lambda(\eta(\cdot-\varepsilon)-\eta(\cdot)) 1_{[-T, 0[}\right)\right.\right. \\
& \left.\left.-D_{x}^{2, \operatorname{Diag}} \mathscr{U}(\eta)\right) \frac{(\eta(x)-\eta(x-\varepsilon))^{2}}{\varepsilon} d x\right) d \lambda \text {. }
\end{aligned}
$$

Firstly, we shall prove that

$$
I_{2}(\varepsilon) \stackrel{\varepsilon \rightarrow 0^{+}}{\longrightarrow} 0
$$

To this end, for every $\varepsilon>0$, we define the operator $T_{\varepsilon}: L^{2}\left([-T, 0]^{2}\right) \rightarrow \mathbb{R}$ as follows:

$$
T_{\varepsilon} g=\int_{[-T, 0]^{2}} g(x, y) \frac{(\eta(x)-\eta(x-\varepsilon))(\eta(y)-\eta(y-\varepsilon))}{\varepsilon} d x d y, \quad \forall g \in L^{2}\left([-T, 0]^{2}\right) .
$$

Then $T_{\varepsilon} \in L^{2}([-T, 0])^{*}$. Indeed, from Cauchy-Schwarz inequality,

$$
\begin{aligned}
\left|T_{\varepsilon} g\right| & \leq\|g\|_{L^{2}\left([-T, 0]^{2}\right)} \sqrt{\int_{[-T, 0]^{2}} \frac{(\eta(x)-\eta(x-\varepsilon))^{2}(\eta(y)-\eta(y-\varepsilon))^{2}}{\varepsilon^{2}} d x d y} \\
& =\|g\|_{L^{2}\left([-T, 0]^{2}\right)} \int_{[-T, 0]} \frac{(\eta(x)-\eta(x-\varepsilon))^{2}}{\varepsilon} d x
\end{aligned}
$$

and the latter quantity is bounded with respect to $\varepsilon$ since the quadratic variation of $\eta$ on $[-T, 0]$ exists. In particular, we have proved that for every $g \in L^{2}\left([-T, 0]^{2}\right)$ there exists a constant $M_{g} \geq 0$ such that

$$
\sup _{0<\varepsilon<1}\left|T_{\varepsilon} g\right| \leq M_{g}
$$

It follows from Banach-Steinhaus theorem that there exists a constant $M \geq 0$ such that

$$
\sup _{0<\varepsilon<1}\left\|T_{\varepsilon}\right\|_{L^{2}([-T, 0])^{*}} \leq M
$$

Now, let us consider the set $\mathscr{S}:=\left\{g \in L^{2}\left([-T, 0]^{2}\right): g(x, y)=e(x) f(y)\right.$, with $\left.e, f \in C^{1}([-T, 0])\right\}$, which is dense in $L^{2}\left([-T, 0]^{2}\right)$. Let us show that

$$
T_{\varepsilon} g \stackrel{\varepsilon \rightarrow 0^{+}}{\longrightarrow} 0, \quad \forall g \in \mathscr{S}
$$


Fix $g \in \mathscr{S}$, with $g(x, y)=e(x) f(y)$ for any $(x, y) \in[-T, 0]$, then

$$
T_{\varepsilon} g=\frac{1}{\varepsilon} \int_{[-T, 0]} e(x)(\eta(x)-\eta(x-\varepsilon)) d x \int_{[-T, 0]} f(y)(\eta(y)-\eta(y-\varepsilon)) d y .
$$

We have

$$
\begin{aligned}
& \left|\int_{[-T, 0]} e(x)(\eta(x)-\eta(x-\varepsilon)) d x\right|=\mid \int_{[-T, 0]}(e(x)-e(x+\varepsilon)) \eta(x) d x \\
& -\int_{[-T-\varepsilon,-T]} e(x+\varepsilon) \eta(x) d x+\int_{[-\varepsilon, 0]} e(x+\varepsilon) \eta(x) d x \mid \\
& \leq \varepsilon\left(\int_{[-T, 0]}|\dot{e}(x)| d x+2\|e\|_{\infty}\right)\|\eta\|_{\infty} .
\end{aligned}
$$

Similarly,

$$
\left|\int_{[-T, 0]} f(y)(\eta(y)-\eta(y-\varepsilon)) d y\right| \leq \varepsilon\left(\int_{[-T, 0]}|\dot{f}(y)| d y+2\|f\|_{\infty}\right)\|\eta\|_{\infty} .
$$

Therefore, from (41) we find

$$
\left|T_{\varepsilon} g\right| \leq \varepsilon\left(\int_{[-T, 0]}|\dot{e}(x)| d x+2\|e\|_{\infty}\right)\left(\int_{[-T, 0]}|\dot{f}(y)| d y+2\|f\|_{\infty}\right)\|\eta\|_{\infty}^{2},
$$

which converges to zero as $\varepsilon$ goes to zero and therefore (40) is established. This in turn implies that

$$
T_{\varepsilon} g \stackrel{\varepsilon \rightarrow 0^{+}}{\longrightarrow} 0, \quad \forall g \in L^{2}\left([-T, 0]^{2}\right) .
$$

Indeed, fix $g \in L^{2}\left([-T, 0]^{2}\right)$ and let $\left(g_{n}\right)_{n} \subset \mathscr{S}$ be such that $g_{n} \rightarrow g$ in $L^{2}\left([-T, 0]^{2}\right)$. Then

$\left|T_{\varepsilon} g\right| \leq\left|T_{\varepsilon}\left(g-g_{n}\right)\right|+\left|T_{\varepsilon} g_{n}\right| \leq\left\|T_{\varepsilon}\right\|_{L^{2}\left([-T, 0]^{2}\right)^{*}}\left\|g-g_{n}\right\|_{L^{2}\left([-T, 0]^{2}\right)}+\left|T_{\varepsilon} g_{n}\right|$.

From (39) it follows that

$$
\left|T_{\varepsilon} g\right| \leq M\left\|g-g_{n}\right\|_{L^{2}\left([-T, 0]^{2}\right)}+\left|T_{\varepsilon} g_{n}\right|
$$

which implies $\lim \sup _{\varepsilon \rightarrow 0^{+}}\left|T_{\varepsilon} g\right| \leq M\left\|g-g_{n}\right\|_{L^{2}\left([-T, 0]^{2}\right)}$. Sending $n$ to infinity, we deduce (42) and finally (38).

Let us now consider the term $I_{3}(\varepsilon)$ in (37). Since the quadratic variation $[\eta]$ exists, it follows from Portmanteau's theorem and hypothesis (i) that 


$$
I_{3}(\varepsilon)=\int_{[-T, 0]} D_{x}^{2, \operatorname{Diag}} \mathscr{U}(\eta) \frac{(\eta(x)-\eta(x-\varepsilon))^{2}}{\varepsilon} d x \underset{\varepsilon \rightarrow 0^{+}}{\longrightarrow} \int_{[-T, 0]} D_{x}^{2, \operatorname{Diag}} \mathscr{U}(\eta) d[\eta](x) .
$$

Regarding the term $I_{4}(\varepsilon)$ in (37), let $\phi_{\eta}:[0,1]^{2} \rightarrow L^{2}\left([-T, 0]^{2}\right)$ be given by

$$
\phi_{\eta}(\varepsilon, \lambda)(\cdot, \cdot)=D_{. .}^{2, L^{2}} u\left(\eta+\lambda(\eta(\cdot-\varepsilon)-\eta(\cdot)) 1_{[-T, 0[}\right) .
$$

By hypothesis, $\phi_{\eta}$ is a continuous map, and hence it is uniformly continuous, since $[0,1]^{2}$ is a compact set. Let $\rho_{\phi_{\eta}}$ denote the continuity modulus of $\phi_{\eta}$, then

$$
\begin{aligned}
& \left\|D_{. .}^{2, L^{2}} u\left(\eta+\lambda(\eta(\cdot-\varepsilon)-\eta(\cdot)) 1_{[-T, 0[}\right)-D_{. .}^{2, L^{2}} \mathscr{U}(\eta)\right\|_{L^{2}\left([-T, 0]^{2}\right)} \\
& =\left\|\phi_{\eta}(\varepsilon, \lambda)-\phi_{\eta}(0, \lambda)\right\|_{L^{2}\left([-T, 0]^{2}\right)} \leq \rho_{\phi_{\eta}}(\varepsilon) .
\end{aligned}
$$

This implies, by Cauchy-Schwarz inequality,

$$
\begin{aligned}
& \mid \int_{0}^{1}(1-\lambda)\left(\int _ { [ - T , 0 ] ^ { 2 } } \left(D_{x y}^{2, L^{2}} u\left(\eta+\lambda(\eta(\cdot-\varepsilon)-\eta(\cdot)) 1_{[-T, 0[}\right)\right.\right. \\
& \left.\left.-D_{x y}^{2, L^{2}} \mathscr{U}(\eta)\right) \frac{(\eta(x)-\eta(x-\varepsilon))(\eta(y)-\eta(y-\varepsilon))}{\varepsilon} d x d y\right) d \lambda \mid \\
& \leq \int_{0}^{1}(1-\lambda) \| D_{. .}^{2, L^{2}} u\left(\eta+\lambda(\eta(\cdot-\varepsilon)-\eta(\cdot)) 1_{[-T, 0]}\right) \\
& -D_{. \cdot}^{2, L^{2}} \mathscr{U}(\eta) \|_{L^{2}\left([-T, 0]^{2}\right)} \sqrt{\int_{[-T, 0]^{2}} \frac{(\eta(x)-\eta(x-\varepsilon))^{2}(\eta(y)-\eta(y-\varepsilon))^{2}}{\varepsilon^{2}} d x d y d \lambda} \\
& \leq \int_{0}^{1}(1-\lambda) \rho_{\phi_{\eta}}(\varepsilon)\left(\int_{[-T, 0]} \frac{(\eta(x)-\eta(x-\varepsilon))^{2}}{\varepsilon} d x\right) d \lambda \\
& =\frac{1}{2} \rho_{\phi_{\eta}}(\varepsilon) \int_{[-T, 0]} \frac{(\eta(x)-\eta(x-\varepsilon))^{2}}{\varepsilon} d x \stackrel{\varepsilon \rightarrow 0^{+}}{\longrightarrow} 0 .
\end{aligned}
$$

Finally, we consider the term $I_{5}(\varepsilon)$ in (37). Define $\psi_{\eta}:[0,1]^{2} \rightarrow L^{\infty}([-T, 0])$ as follows:

$$
\psi_{\eta}(\varepsilon, \lambda)(\cdot)=D^{2,{ }^{D i a g}} u\left(\eta+\lambda(\eta(\cdot-\varepsilon)-\eta(\cdot)) 1_{[-T, 0[}\right) .
$$

We see that $\psi_{\eta}$ is uniformly continuous. Let $\rho_{\psi_{\eta}}$ denote the continuity modulus of $\psi_{\eta}$, then

$$
\begin{aligned}
& \left\|D^{2,{ }^{, D i a g}} u\left(\eta+\lambda(\eta(\cdot-\varepsilon)-\eta(\cdot)) 1_{[-T, 0[}\right)-D_{.}^{2,{ }^{\text {Diag }}} \mathscr{U}(\eta)\right\|_{L^{\infty}([-T, 0])} \\
& =\left\|\psi_{\eta}(\varepsilon, \lambda)-\psi_{\eta}(0, \lambda)\right\|_{L^{\infty}([-T, 0])} \leq \rho_{\psi_{\eta}}(\varepsilon) \text {. }
\end{aligned}
$$


Therefore, we have

$$
\begin{aligned}
& \mid \int_{0}^{1}(1-\lambda)\left(\int _ { [ - T , 0 ] } \left(D_{x}^{2, D i a g} u\left(\eta+\lambda(\eta(\cdot-\varepsilon)-\eta(\cdot)) 1_{[-T, 0[}\right)\right.\right. \\
& \left.\left.-D_{x}^{2, \operatorname{Diag}} \mathscr{U}(\eta)\right) \frac{(\eta(x)-\eta(x-\varepsilon))^{2}}{\varepsilon} d x\right) d \lambda \mid \\
& \leq \int_{0}^{1}(1-\lambda)\left(\int_{[-T, 0]} \rho_{\psi_{\eta}}(\varepsilon) \frac{(\eta(x)-\eta(x-\varepsilon))^{2}}{\varepsilon} d x\right) d \lambda \\
& =\frac{1}{2} \rho_{\psi_{\eta}}(\varepsilon) \int_{[-T, 0]} \frac{(\eta(x)-\eta(x-\varepsilon))^{2}}{\varepsilon} d x \stackrel{\varepsilon \rightarrow 0^{+}}{\longrightarrow} 0 .
\end{aligned}
$$

In conclusion, we have proved that all the integral terms in the right-hand side of (37), unless $I_{1}(\varepsilon)$, admit a limit when $\varepsilon$ goes to zero. Since the left-hand side admits a limit, namely $D^{H} \mathscr{U}(\eta)$, we deduce that the backward integral

$$
I_{1}(\varepsilon)=\int_{[-T, 0]} D_{d x}^{\perp} \mathscr{U}(\eta) \frac{\eta(x)-\eta(x-\varepsilon)}{\varepsilon} \stackrel{\varepsilon \rightarrow 0^{+}}{\longrightarrow} \int_{[-T, 0]} D_{d x}^{\perp} \mathscr{U}(\eta) d^{+} \eta(x)
$$

exists and it is finite, which concludes the proof.

\section{Strong-Viscosity Solutions to Path-Dependent PDEs}

In the present section we study the semilinear parabolic path-dependent equation

$$
\begin{cases}\partial_{t} \mathscr{U}+D^{H} \mathscr{U}+\frac{1}{2} D^{V V} \mathscr{U}+F\left(t, \eta, \mathscr{U}, D^{V} \mathscr{U}\right)=0, & \forall(t, \eta) \in[0, T[\times C([-T, 0]), \\ \mathscr{U}(T, \eta)=H(\eta), & \forall \eta \in C([-T, 0]) .\end{cases}
$$

We refer to $\mathscr{L} \mathscr{U}=\partial_{t} \mathscr{U}+D^{H} \mathscr{U}+\frac{1}{2} D^{V V} \mathscr{U}$ as the path-dependent heat operator. The results of this section are generalized in $[9,10]$, where more general path-dependent equations will be considered. Here we shall impose the following assumptions on $H$ and $F$.

(A) $H: C([-T, 0]) \rightarrow \mathbb{R}$ and $F:[0, T] \times C([-T, 0]) \times \mathbb{R} \times \mathbb{R} \rightarrow \mathbb{R}$ are Borel measurable functions and satisfy, for some positive constants $C$ and $m$,

$$
\begin{aligned}
\left|F(t, \eta, y, z)-F\left(t, \eta, y^{\prime}, z^{\prime}\right)\right| & \leq C\left(\left|y-y^{\prime}\right|+\left|z-z^{\prime}\right|\right), \\
|H(\eta)|+|F(t, \eta, 0,0)| & \leq C\left(1+\|\eta\|_{\infty}^{m}\right)
\end{aligned}
$$

for all $(t, \eta) \in[0, T] \times C([-T, 0]), y, y^{\prime} \in \mathbb{R}$, and $z, z^{\prime} \in \mathbb{R}$. 


\subsection{Strict Solutions}

In the present subsection, we provide the definition of strict solution to Eq. (43) and we state an existence and uniqueness result.

Definition 19 A function $\mathscr{U}:[0, T] \times C([-T, 0]) \rightarrow \mathbb{R}$ in $C^{1,2}(([0, T[\times$ past $) \times$ present $) \cap C([0, T] \times C([-T, 0]))$, which solves Eq. (43), is called a strict solution to (43).

We now introduce some additional notations. Let $(\Omega, \mathscr{F}, \mathbb{P})$ be a complete probability space on which a real Brownian motion $W=\left(W_{t}\right)_{t \geq 0}$ is defined. Let $\mathbb{F}=\left(\mathscr{F}_{t}\right)_{t \geq 0}$ denote the completion of the natural filtration generated by $W$.

- $\mathbb{S}^{p}(t, T), p \geq 1,0 \leq t \leq T$, the set of real càdlàg $\mathbb{F}$-adapted processes $Y=$ $\left(Y_{s}\right)_{t \leq s \leq T}$ such that

$$
\|Y\|_{\mathbb{S} p(t, T)}^{p}:=\mathbb{E}\left[\sup _{t \leq s \leq T}\left|Y_{S}\right|^{p}\right]<\infty
$$

- $\mathbb{H}^{p}(t, T)^{d}, p \geq 1,0 \leq t \leq T$, the set of $\mathbb{R}^{d}$-valued predictable processes $Z=$ $\left(Z_{S}\right)_{t \leq s \leq T}$ such that

$$
\|Z\|_{\mathbb{H}^{p}(t, T)^{d}}^{p}:=\mathbb{E}\left[\left(\int_{t}^{T}\left|Z_{s}\right|^{2} d s\right)^{\frac{p}{2}}\right]<\infty .
$$

We simply write $\mathbb{H}^{p}(t, T)$ when $d=1$.

- $\mathbb{A}^{+, 2}(t, T), 0 \leq t \leq T$, the set of real nondecreasing predictable processes $K=$ $\left(K_{s}\right)_{t \leq s \leq T} \in \mathbb{S}^{2}(t, T)$ with $K_{t}=0$, so that

$$
\|K\|_{\mathbb{S}^{2}(t, T)}^{2}:=\mathbb{E}\left[\left|K_{T}\right|^{2}\right]
$$

- $\mathbb{L}^{p}\left(t, T ; \mathbb{R}^{m}\right), p \geq 1,0 \leq t \leq T$, the set of $\mathbb{R}^{m}$-valued $\mathbb{F}$-predictable processes $\phi=\left(\phi_{s}\right)_{t \leq s \leq T}$ such that

$$
\|\phi\|_{\mathbb{L}^{p}\left(t, T ; \mathbb{R}^{m}\right)}^{p}:=\mathbb{E}\left[\int_{t}^{T}\left|\phi_{s}\right|^{p} d s\right]<\infty .
$$

Definition 20 Let $t \in[0, T]$ and $\eta \in C([-T, 0])$. Then, we define the stochastic flow

$$
\mathbb{W}_{s}^{t, \eta}(x)= \begin{cases}\eta(x+s-t), & -T \leq x \leq t-s, \\ \eta(0)+W_{x+s}-W_{t}, & t-s<x \leq 0,\end{cases}
$$

for any $t \leq s \leq T$. 
Theorem 4 Suppose that Assumption (A) holds. Let $\mathscr{U}:[0, T] \times C([-T, 0]) \rightarrow \mathbb{R}$ be a strict solution to Eq. (43), satisfying the polynomial growth condition

$$
|\mathscr{U}(t, \eta)| \leq C\left(1+\|\eta\|_{\infty}^{m}\right), \quad \forall(t, \eta) \in[0, T] \times C([-T, 0]) .
$$

for some positive constants $C$ and $m$. Then, we have

$$
\mathscr{U}(t, \eta)=Y_{t}^{t, \eta}, \quad \forall(t, \eta) \in[0, T] \times C([-T, 0]),
$$

where $\left(Y_{s}^{t, \eta}, Z_{s}^{t, \eta}\right)_{s \in[t, T]}=\left(\mathscr{U}\left(s, \mathbb{W}_{s}^{t, \eta}\right), D^{V} \mathscr{U}\left(s, \mathbb{W}_{s}^{t, \eta}\right) 1_{[t, T[}(s)\right)_{s \in[t, T]} \in \mathbb{S}^{2}$ $(t, T) \times \mathbb{H}^{2}(t, T)$ is the solution to the backward stochastic differential equation: $\mathbb{P}$-a.s.,

$$
Y_{s}^{t, \eta}=H\left(\mathbb{W}_{T}^{t, \eta}\right)+\int_{s}^{T} F\left(r, \mathbb{W}_{r}^{t, \eta}, Y_{r}^{t, \eta}, Z_{r}^{t, \eta}\right) d r-\int_{s}^{T} Z_{r}^{t, \eta} d W_{r}, \quad t \leq s \leq T
$$

In particular, there exists at most one strict solution to Eq. (43).

Proof Fix $(t, \eta) \in[0, T[\times C([-T, 0])$ and set, for all $t \leq s \leq T$,

$$
Y_{s}^{t, \eta}=\mathscr{U}\left(s, \mathbb{W}_{s}^{t, \eta}\right), \quad Z_{s}^{t, \eta}=D^{V} \mathscr{U}\left(s, \mathbb{W}_{s}^{t, \eta}\right) 1_{[t, T[}(s) .
$$

Then, for any $T_{0} \in\left[t, T\right.$, applying Itô formula (28) to $\mathscr{U}\left(s, \mathbb{W}_{s}^{t, \eta}\right)$ and using the fact that $\mathscr{U}$ solves Eq. (43), we find, $\mathbb{P}$-a.s.,

$$
Y_{s}^{t, \eta}=Y_{T_{0}}^{t, \eta}+\int_{s}^{T_{0}} F\left(r, \mathbb{W}_{r}^{t, \eta}, Y_{r}^{t, \eta}, Z_{r}^{t, \eta}\right) d r-\int_{s}^{T_{0}} Z_{r}^{t, \eta} d W_{r}, \quad t \leq s \leq T_{0} .
$$

The claim would follow if we could pass to the limit in (45) as $T_{0} \rightarrow T$. To do this, we notice that it follows from Proposition B.1 in [10] that there exists a positive constant $c$, depending only on $T$ and the constants $C$ and $m$ appearing in the statement of the present Theorem 4, such that

$\mathbb{E} \int_{t}^{T_{0}}\left|Z_{s}^{t, \eta}\right|^{2} d s \leq c\left\|Y^{t, \eta}\right\|_{\mathbb{S}^{2}(t, T)}^{2}+c \mathbb{E} \int_{t}^{T}\left|F\left(r, \mathbb{W}_{r}^{t, \eta}, 0,0\right)\right|^{2} d r, \quad \forall T_{0} \in[t, T[$.

We recall that, for any $q \geq 1$,

$$
\mathbb{E}\left[\sup _{t \leq s \leq T}\left\|\mathbb{W}_{s}^{t, \eta}\right\|_{\infty}^{q}\right]<\infty
$$

Notice that from (44) and (46) we have $\left\|Y^{t, \eta}\right\|_{\mathbb{S}^{2}(t, T)}<\infty$, so that $Y \in \mathbb{S}^{2}(t, T)$. Then, from the monotone convergence theorem we find 


$$
\mathbb{E} \int_{t}^{T}\left|Z_{s}^{t, \eta}\right|^{2} d s \leq c\left\|Y^{t, \eta}\right\|_{\mathbb{S}^{2}(t, T)}^{2}+c \mathbb{E} \int_{t}^{T}\left|F\left(r, \mathbb{W}_{r}^{t, \eta}, 0,0\right)\right|^{2} d r
$$

Therefore, it follows from the polynomial growth condition of $F$ and (46) that $Z \in \mathbb{H}^{2}(t, T)$. This implies, using the Lipschitz character of $F$ in $(y, z)$, that $\mathbb{E} \int_{t}^{T}\left|F\left(r, \mathbb{W}_{r}^{t, \eta}, Y_{r}^{t, \eta}, Z_{r}^{t, \eta}\right)\right|^{2} d r<\infty$, so that we can pass to the limit in (45) and we get the claim.

We conclude this subsection with an existence result for the path-dependent heat equation, namely for Eq. (43) with $F \equiv 0$, for which we provide an ad hoc proof. For more general cases we refer to [9].

Theorem 5 Suppose that Assumption (A) holds. Let $F \equiv 0$ and $H$ be given by, for all $\eta \in C([-T, 0])$, (the deterministic integrals are defined according to Definition $4(i))$

$$
H(\eta)=h\left(\int_{[-T, 0]} \varphi_{1}(x+T) d^{-} \eta(x), \ldots, \int_{[-T, 0]} \varphi_{N}(x+T) d^{-} \eta(x)\right),
$$

where

- $h$ belongs $C^{2}\left(\mathbb{R}^{N}\right)$ and its second order partial derivatives satisfy a polynomial growth condition,

- $\varphi_{1}, \ldots, \varphi_{N} \in C^{2}([0, T])$.

Then, there exists a unique strict solution $\mathscr{U}$ to the path-dependent heat Eq. (43), which is given by

$$
\mathscr{U}(t, \eta)=\mathbb{E}\left[H\left(\mathbb{W}_{T}^{t, \eta}\right)\right], \quad \forall(t, \eta) \in[0, T] \times C([-T, 0]) .
$$

Proof Let us consider the function $\mathscr{U}:[0, T] \times C([-T, 0]) \rightarrow \mathbb{R}$ given by, for all $(t, \eta) \in[0, T] \times C([-T, 0])$,

$$
\begin{aligned}
\mathscr{U}(t, \eta) & =\mathbb{E}\left[H\left(\mathbb{W}_{T}^{t, \eta}\right)\right] \\
& =\mathbb{E}\left[h\left(\int_{[-t, 0]} \varphi_{1}(x+t) d^{-} \eta(x)+\int_{t}^{T} \varphi_{1}(s) d W_{s}, \ldots\right)\right] \\
& =\Psi\left(t, \int_{[-t, 0]} \varphi_{1}(x+t) d^{-} \eta(x), \ldots, \int_{[-t, 0]} \varphi_{N}(x+t) d^{-} \eta(x)\right),
\end{aligned}
$$

where

$$
\Psi\left(t, x_{1}, \ldots, x_{N}\right)=\mathbb{E}\left[h\left(x_{1}+\int_{t}^{T} \varphi_{1}(s) d W_{s}, \ldots, x_{N}+\int_{t}^{T} \varphi_{N}(s) d W_{s}\right)\right],
$$


for any $\left(t, x_{1}, \ldots, x_{N}\right) \in[0, T] \times \mathbb{R}^{N}$. Notice that, for any $i, j=1, \ldots, N$,

$$
\begin{aligned}
D_{x_{i}} \Psi\left(t, x_{1}, \ldots, x_{N}\right) & =\mathbb{E}\left[D_{x_{i}} h\left(x_{1}+\int_{t}^{T} \varphi_{1}(s) d W_{s}, \ldots, x_{N}+\int_{t}^{T} \varphi_{N}(s) d W_{s}\right)\right], \\
D_{x_{i} x_{j}}^{2} \Psi\left(t, x_{1}, \ldots, x_{N}\right) & =\mathbb{E}\left[D_{x_{i} x_{j}}^{2} h\left(x_{1}+\int_{t}^{T} \varphi_{1}(s) d W_{s}, \ldots, x_{N}+\int_{t}^{T} \varphi_{N}(s) d W_{s}\right)\right],
\end{aligned}
$$

so that $\Psi$ and its first and second spatial derivatives are continuous on $[0, T] \times \mathbb{R}^{N}$. Let us focus on the time derivative $\partial_{t} \Psi$ of $\Psi$. We have, for any $\delta>0$ such that $t+\delta \in[0, T]$,

$$
\begin{aligned}
& \Psi\left(t+\delta, x_{1}, \ldots, x_{N}\right)-\Psi\left(t, x_{1}, \ldots, x_{N}\right) \\
& =\frac{1}{\delta} \mathbb{E}\left[h\left(x_{1}+\int_{t+\delta}^{T} \varphi_{1}(s) d W_{s}, \ldots\right)-h\left(x_{1}+\int_{t}^{T} \varphi_{1}(s) d W_{s}, \ldots\right)\right] .
\end{aligned}
$$

Then, using a standard Taylor formula, we find

$$
\begin{aligned}
& \Psi\left(t+\delta, x_{1}, \ldots, x_{N}\right)-\Psi\left(t, x_{1}, \ldots, x_{N}\right) \\
& \delta \\
& =-\frac{1}{\delta} \mathbb{E}\left[\int_{0}^{1} \sum_{i=1}^{N} D_{x_{i}} h\left(x_{1}+\int_{t}^{T} \varphi_{1}(s) d W_{s}-\alpha \int_{t}^{t+\delta} \varphi_{1}(s) d W_{s}, \ldots\right) \int_{t}^{t+\delta} \varphi_{i}(s) d W_{s} d \alpha\right] .
\end{aligned}
$$

Now, it follows from the integration by parts formula of Malliavin calculus, see, e.g., formula (1.42) in [31] (taking into account that Itô integrals are Skorohod integrals), that, for any $i=1, \ldots, N$,

$$
\begin{aligned}
& \mathbb{E}\left[D_{x_{i}} h\left(x_{1}+\int_{t}^{T} \varphi_{1}(s)\left(1-\alpha 1_{[t, t+\delta]}(s)\right) d W_{s}, \ldots\right) \int_{t}^{t+\delta} \varphi_{i}(s) d W_{s}\right] \\
& =(1-\alpha) \mathbb{E}\left[\sum_{j=1}^{N} D_{x_{i} x_{j}}^{2} h\left(x_{1}+\int_{t}^{T} \varphi_{1}(s)\left(1-\alpha 1_{[t, t+\delta]}(s)\right) d W_{s}, \ldots\right) \int_{t}^{t+\delta} \varphi_{i}(s) \varphi_{j}(s) d s\right] .
\end{aligned}
$$

Then, plugging (49) into (48) and letting $\delta \rightarrow 0^{+}$, we get (recalling that $D_{x_{i} x_{j}}^{2} h$ has polynomial growth, for any $i, j$ )

$$
\partial_{t}^{+} \Psi\left(t, x_{1}, \ldots, x_{N}\right)=-\frac{1}{2} \mathbb{E}\left[\sum_{i, j=1}^{N} D_{x_{i} x_{j}}^{2} h\left(x_{1}+\int_{t}^{T} \varphi_{1}(s) d W_{s}, \ldots\right) \varphi_{i}(t) \varphi_{j}(t)\right]
$$

for any $\left(t, x_{1}, \ldots, x_{N}\right) \in\left[0, T\left[\times \mathbb{R}^{N}\right.\right.$, where $\partial_{t}^{+} \Psi$ denotes the right-time derivative of $\Psi$. Since $\Psi$ and $\partial_{t}^{+} \Psi$ are continuous, we deduce that $\partial_{t} \Psi$ exists and is continuous on $[0, T[$ (see for example Corollary 1.2, Chap.2, in [32]). Moreover, from the 
representation formula (50) we see that $\partial_{t} \Psi$ exists and is continuous up to time $T$. Furthermore, from the expression of $D_{x_{i} x_{j}}^{2} \Psi$, we see that

$$
\partial_{t} \Psi\left(t, x_{1}, \ldots, x_{N}\right)=-\frac{1}{2} \sum_{i, j=1}^{N} \varphi_{i}(t) \varphi_{j}(t) D_{x_{i} x_{j}}^{2} \Psi\left(t, x_{1}, \ldots, x_{N}\right)
$$

Therefore, $\Psi \in C^{1,2}\left([0, T] \times \mathbb{R}^{N}\right)$ and is a classical solution to the Cauchy problem

$$
\begin{cases}\partial_{t} \Psi(t, \mathbf{x})+\frac{1}{2} \sum_{i, j=1}^{N} \varphi_{i}(t) \varphi_{j}(t) D_{x_{i} x_{j}}^{2} \Psi(t, \mathbf{x})=0, & \forall(t, \mathbf{x}) \in\left[0, T\left[\times \mathbb{R}^{N},\right.\right. \\ \Psi(T, \mathbf{x})=h(\mathbf{x}), & \forall \mathbf{x} \in \mathbb{R}^{N} .\end{cases}
$$

Now we express the derivatives of $\mathscr{U}$ in terms of $\Psi$. We begin noting that, taking into account Proposition 4, we have

$$
\int_{[-t, 0]} \varphi_{i}(x+t) d^{-} \eta(x)=\eta(0) \varphi_{i}(t)-\int_{-t}^{0} \eta(x) \dot{\varphi}_{i}(x+t) d x, \quad \forall \eta \in C([-T, 0]) .
$$

This in turn implies that $\mathscr{U}$ is continuous with respect to the topology of $\mathscr{C}([-T, 0])$. Therefore, $\mathscr{U}$ admits a unique extension $u: \mathscr{C}([-T, 0]) \rightarrow \mathbb{R}$, which is given by

$$
u(t, \eta)=\Psi\left(t, \int_{[-t, 0]} \varphi_{1}(x+t) d^{-} \eta(x), \ldots, \int_{[-t, 0]} \varphi_{N}(x+t) d^{-} \eta(x)\right),
$$

for all $(t, \eta) \in[0, T] \times \mathscr{C}([-T, 0])$. We also define the map $\tilde{u}:[0, T] \times \mathscr{C}([-T, 0[) \times$ $\mathbb{R} \rightarrow \mathbb{R}$ as in (13):

$$
\tilde{u}(t, \gamma, a)=u\left(t, \gamma 1_{[-T, 0[}+a 1_{\{0\}}\right)=\Psi\left(t, \ldots, a \varphi_{i}(t)-\int_{-t}^{0} \gamma(x) \dot{\varphi}_{i}(x+t) d x, \ldots\right),
$$

for all $(t, \gamma, a) \in[0, T] \times \mathscr{C}([-T, 0[) \times \mathbb{R}$. Let us evaluate the time derivative $\partial_{t} \mathscr{U}(t, \eta)$, for a given $(t, \eta) \in[0, T[\times C([-T, 0])$ :

$$
\begin{aligned}
\partial_{t} \mathscr{U}(t, \eta)= & \partial_{t} \Psi\left(t, \int_{[-t, 0]} \varphi_{1}(x+t) d^{-} \eta(x), \ldots, \int_{[-t, 0]} \varphi_{N}(x+t) d^{-} \eta(x)\right) \\
& +\sum_{i=1}^{N} D_{x_{i}} \Psi\left(t, \ldots, \int_{[-t, 0]} \varphi_{i}(x+t) d^{-} \eta(x), \ldots\right) \partial_{t}\left(\int_{[-t, 0]} \varphi_{i}(x+t) d^{-} \eta(x)\right) .
\end{aligned}
$$

Notice that

$$
\begin{aligned}
\partial_{t}\left(\int_{[-t, 0]} \varphi_{i}(x+t) d^{-} \eta(x)\right) & =\partial_{t}\left(\eta(0) \varphi(t)-\int_{-t}^{0} \eta(x) \dot{\varphi}_{i}(x+t) d x\right) \\
& =\eta(0) \dot{\varphi}(t)-\eta(-t) \dot{\varphi}_{i}\left(0^{+}\right)-\int_{-t}^{0} \eta(x) \ddot{\varphi}_{i}(x+t) d x .
\end{aligned}
$$


Functional and Banach Space Stochastic Calculi ...

67

Let us proceed with the horizontal derivative. We have

$$
\begin{aligned}
& D^{H} \mathscr{U}(t, \eta)=D^{H} u(t, \eta)=D^{H} \tilde{u}\left(t, \eta_{\mid[-T, 0[}, \eta(0)\right) \\
& =\lim _{\varepsilon \rightarrow 0^{+}} \frac{\tilde{u}\left(t, \eta_{\mid[-T, 0[}(\cdot), \eta(0)\right)-\tilde{u}\left(t, \eta_{\mid[-T, 0[}(\cdot-\varepsilon), \eta(0)\right)}{\varepsilon} \\
& =\lim _{\varepsilon \rightarrow 0^{+}}\left(\frac{1}{\varepsilon} \Psi\left(t, \ldots, \eta(0) \varphi_{i}(t)-\int_{-t}^{0} \eta(x) \dot{\varphi}_{i}(x+t) d x, \ldots\right)\right. \\
& \left.\quad-\frac{1}{\varepsilon} \Psi\left(t, \ldots, \eta(0) \varphi_{i}(t)-\int_{-t}^{0} \eta(x-\varepsilon) \dot{\varphi}_{i}(x+t) d x, \ldots\right)\right) .
\end{aligned}
$$

From the fundamental theorem of calculus, we obtain

$$
\begin{aligned}
& \frac{1}{\varepsilon} \Psi\left(t, \ldots, \eta(0) \varphi_{i}(t)-\int_{-t}^{0} \eta(x) \dot{\varphi}_{i}(x+t) d x, \ldots\right) \\
& -\frac{1}{\varepsilon} \Psi\left(t, \ldots, \eta(0) \varphi_{i}(t)-\int_{-t}^{0} \eta(x-\varepsilon) \dot{\varphi}_{i}(x+t) d x, \ldots\right) \\
& =\frac{1}{\varepsilon} \int_{0}^{\varepsilon} \sum_{i=1}^{N} D_{x_{i}} \Psi\left(t, \ldots, \eta(0) \varphi_{i}(t)-\int_{-t}^{0} \eta(x-y) \dot{\varphi}_{i}(x+t) d x, \ldots\right) \partial_{y}\left(\eta(0) \varphi_{i}(t)\right. \\
& \left.\quad-\int_{-t}^{0} \eta(x-y) \dot{\varphi}_{i}(x+t) d x\right) d y .
\end{aligned}
$$

Notice that

$$
\begin{aligned}
& \partial_{y}\left(\eta(0) \varphi_{i}(t)-\int_{-t}^{0} \eta(x-y) \dot{\varphi}_{i}(x+t) d x\right)=-\partial_{y}\left(\int_{-t-y}^{-y} \eta(x) \dot{\varphi}_{i}(x+y+t) d x\right) \\
& =-\left(\eta(-y) \dot{\varphi}_{i}(t)-\eta(-t-y) \dot{\varphi}_{i}\left(0^{+}\right)+\int_{-t-y}^{-y} \eta(x) \ddot{\varphi}_{i}(x+y+t) d x\right) .
\end{aligned}
$$

Therefore

$$
\begin{aligned}
& D^{H} \mathscr{U}(t, \eta) \\
&=-\lim _{\varepsilon \rightarrow 0^{+}} \frac{1}{\varepsilon} \int_{0}^{\varepsilon} \sum_{i=1}^{N} D_{x_{i}} \Psi\left(t, \ldots, \eta(0) \varphi_{i}(t)-\int_{-t}^{0} \eta(x-y) \dot{\varphi}_{i}(x+t) d x, \ldots\right)\left(\eta(-y) \dot{\varphi}_{i}(t)\right. \\
&\left.-\eta(-t-y) \dot{\varphi}_{i}\left(0^{+}\right)+\int_{-t-y}^{-y} \eta(x) \ddot{\varphi}_{i}(x+y+t) d x\right) d y \\
&=-\sum_{i=1}^{N} D_{x_{i}} \Psi\left(t, \ldots, \eta(0) \varphi_{i}(t)-\int_{-t}^{0} \eta(x) \dot{\varphi}_{i}(x+t) d x, \ldots\right)\left(\eta(0) \dot{\varphi}(t)-\eta(-t) \dot{\varphi}_{i}\left(0^{+}\right)\right. \\
&\left.-\int_{-t}^{0} \eta(x) \ddot{\varphi}_{i}(x+t) d x\right) .
\end{aligned}
$$


Finally, concerning the vertical derivative we have

$$
\begin{aligned}
D^{V} \mathscr{U}(t, \eta)=D^{V} u(t, \eta) & =\partial_{a} \tilde{u}\left(t, \eta 1_{[-T, 0[}+\eta(0) 1_{\{0\}}\right) \\
& =\sum_{i=1}^{N} D_{x_{i}} \Psi\left(t, \int_{[-t, 0]} \varphi_{1}(x+t) d^{-} \eta(x), \ldots\right) \varphi_{i}(t)
\end{aligned}
$$

and

$$
\begin{aligned}
D^{V V} \mathscr{U}(t, \eta)=D^{V V} u(t, \eta) & =\partial_{a a}^{2} \tilde{u}\left(t, \eta 1_{[-T, 0[}+\eta(0) 1_{\{0\}}\right) \\
& =\sum_{i, j=1}^{N} D_{x_{i} x_{j}}^{2} \Psi\left(t, \int_{[-t, 0]} \varphi_{1}(x+t) d^{-} \eta(x), \ldots\right) \varphi_{i}(t) \varphi_{j}(t) .
\end{aligned}
$$

From the regularity of $\Psi$ it follows that $\mathscr{U} \in C^{1,2}(([0, T] \times$ past $) \times$ present $)$. Moreover, since $\Psi$ satisfies the Cauchy problem (51), we conclude that $\partial_{t} \mathscr{U}(t, \eta)+$ $D^{H} \mathscr{U}(t, \eta)+\frac{1}{2} D^{V V} \mathscr{U}(t, \eta)=0$, for all $(t, \eta) \in[0, T[\times C([-T, 0])$, therefore $\mathscr{U}$ is a classical solution to the path-dependent heat Eq. (43).

\subsection{Towards a Weaker Notion of Solution: A Significant Hedging Example}

In the present subsection, we consider Eq. (43) in the case $F \equiv 0$. This situation is particularly interesting, since it arises, for example, in hedging problems of path-dependent contingent claims. More precisely, consider a real continuous finite quadratic variation process $X$ on $(\Omega, \mathscr{F}, \mathbb{P})$ and denote $\mathbb{X}$ the window process associated to $X$. Let us assume that $[X]_{t}=t$, for any $t \in[0, T]$. The hedging problem that we have in mind is the following: given a contingent claim's payoff $H\left(\mathbb{X}_{T}\right)$, is it possible to have

$$
H\left(\mathbb{X}_{T}\right)=H_{0}+\int_{0}^{T} Z_{t} d^{-} X_{t},
$$

for some $H_{0} \in \mathbb{R}$ and some $\mathbb{F}$-adapted process $Z=\left(Z_{t}\right)_{t \in[0, T]}$ such that $Z_{t}=$ $v\left(t, \mathbb{X}_{t}\right)$, with $v:[0, T] \times C([-T, 0]) \rightarrow \mathbb{R}$ ? When $X$ is a Brownian motion $W$ and $\int_{0}^{T}\left|Z_{t}\right|^{2} d t<\infty, \mathbb{P}$-a.s., the previous forward integral is an Itô integral. If $H$ is regular enough and it is cylindrical in the sense of (47), we know from Theorem 5 that there exists a unique classical solution $\mathscr{U}:[0, T] \times C([-T, 0]) \rightarrow \mathbb{R}$ to Eq. (43).

Then, we see from Itô's formula (28) that $\mathscr{U}$ satisfies, $\mathbb{P}$-a.s.,

$$
\mathscr{U}\left(t, \mathbb{X}_{t}\right)=\mathscr{U}\left(0, \mathbb{X}_{0}\right)+\int_{0}^{t} D^{V} \mathscr{U}\left(s, \mathbb{X}_{s}\right) d^{-} X_{s}, \quad 0 \leq t \leq T
$$

In particular, (52) holds with $Z_{t}=D^{V} \mathscr{U}\left(t, \mathbb{X}_{t}\right)$, for any $t \in[0, T], H_{0}=\mathscr{U}\left(0, \mathbb{X}_{t}\right)$. 
However, a significant hedging example is the lookback-type payoff

$$
H(\eta)=\sup _{x \in[-T, 0]} \eta(x), \quad \forall \eta \in C([-T, 0]) .
$$

We look again for $\mathscr{U}:[0, T] \times C([-T, 0]) \rightarrow \mathbb{R}$ which verifies (53), at least for $X$ being a Brownian motion $W$. Since $\mathscr{U}\left(t, \mathbb{W}_{t}\right)$ has to be a martingale, a candidate for $\mathscr{U}$ is $\mathscr{U}(t, \eta)=\mathbb{E}\left[H\left(\mathbb{W}_{T}^{t, \eta}\right)\right]$, for all $(t, \eta) \in[0, T] \times C([-T, 0])$. However, this latter $\mathscr{U}$ can be shown not to be regular enough in order to be a classical solution to Eq. (43), even if it is "virtually" a solution to the path-dependent semilinear Kolmogorov equation (43). This will lead us to introduce a weaker notion of solution to Eq. (43). To characterize the map $\mathscr{U}$, we notice that it admits the probabilistic representation formula, for all $(t, \eta) \in[0, T] \times C([-T, 0])$,

$$
\begin{aligned}
\mathscr{U}(t, \eta) & =\mathbb{E}\left[H\left(\mathbb{W}_{T}^{t, \eta}\right)\right]=\mathbb{E}\left[\sup _{-T \leq x \leq 0} \mathbb{W}_{T}^{t, \eta}(x)\right] \\
& =\mathbb{E}\left[\left(\sup _{-t \leq x \leq 0} \eta(x)\right) \vee\left(\sup _{t \leq x \leq T}\left(W_{x}-W_{t}+\eta(0)\right)\right)\right]=f\left(t, \sup _{-t \leq x \leq 0} \eta(x), \eta(0)\right),
\end{aligned}
$$

where the function $f:[0, T] \times \mathbb{R} \times \mathbb{R} \rightarrow \mathbb{R}$ is given by

$$
f(t, m, x)=\mathbb{E}\left[m \vee\left(S_{T-t}+x\right)\right], \quad \forall(t, m, x) \in[0, T] \times \mathbb{R} \times \mathbb{R},
$$

with $S_{t}=\sup _{0 \leq s \leq t} W_{s}$, for all $t \in[0, T]$. Recalling Remark 3, it follows from the presence of $\sup _{-t \leq x \leq 0} \eta(x)$ among the arguments of $f$, that $\mathscr{U}$ is not continuous with respect to the topology of $\mathscr{C}([-T, 0])$, therefore it can not be a classical solution to Eq. (43). However, we notice that $\sup _{-t \leq x \leq 0} \eta(x)$ is Lipschitz on $(C([-T, 0]), \|$. $\left.\|_{\infty}\right)$, therefore it will follow from Theorem 7 that $\mathscr{U}$ is a strong-viscosity solution to Eq. (43) in the sense of Definition 21. Nevertheless, in this particular case, even if $\mathscr{U}$ is not a classical solution, we shall prove that it is associated to the classical solution of a certain finite dimensional PDE. To this end, we begin computing an explicit form for $f$, for which it is useful to recall the following standard result.

Lemma 1 (Reflection principle) For every $a>0$ and $t>0$,

$$
\mathbb{P}\left(S_{t} \geq a\right)=\mathbb{P}\left(\left|B_{t}\right| \geq a\right)
$$

In particular, for each $t$, the random variables $S_{t}$ and $\left|B_{t}\right|$ have the same law, whose density is given by:

$$
\varphi_{t}(z)=\sqrt{\frac{2}{\pi t}} e^{-\frac{z^{2}}{2 t}} 1_{[0, \infty[}(z), \quad \forall z \in \mathbb{R} .
$$


Proof See Proposition 3.7, Chapter III, in [35].

From Lemma 1 it follows that, for all $(t, m, x) \in[0, T[\times \mathbb{R} \times \mathbb{R}$,

$$
f(t, m, x)=\int_{0}^{\infty} m \vee(z+x) \varphi_{T-t}(z) d z=\int_{0}^{\infty} m \vee(z+x) \frac{2}{\sqrt{T-t}} \varphi\left(\frac{z}{\sqrt{T-t}}\right) d z,
$$

where $\varphi(z)=\exp \left(z^{2} / 2\right) / \sqrt{2 \pi}, z \in \mathbb{R}$, is the standard Gaussian density.

Lemma 2 The function $f$ defined in (54) is given by, for all $(t, m, x) \in[0, T[\times \mathbb{R} \times$ $\mathbb{R}$,

$$
f(t, m, x)=2 m\left(\Phi\left(\frac{m-x}{\sqrt{T-t}}\right)-\frac{1}{2}\right)+2 x\left(1-\Phi\left(\frac{m-x}{\sqrt{T-t}}\right)\right)+\sqrt{\frac{2(T-t)}{\pi}} e^{-\frac{(m-x)^{2}}{2(T-t)}},
$$

for $x \leq m$, and

$$
f(t, x, m)=x+\sqrt{\frac{2(T-t)}{\pi}},
$$

for $x>m$, where $\Phi(y)=\int_{-\infty}^{y} \varphi(z) d z, y \in \mathbb{R}$, is the standard Gaussian cumulative distribution function.

Proof First case: $x \leq m$. We have

$$
f(t, m, x)=\int_{0}^{m-x} m \frac{2}{\sqrt{T-t}} \varphi\left(\frac{z}{\sqrt{T-t}}\right) d z+\int_{m-x}^{\infty}(z+x) \frac{2}{\sqrt{T-t}} \varphi\left(\frac{z}{\sqrt{T-t}}\right) d z .
$$

The first integral on the right-hand side of (55) becomes

$\int_{0}^{m-x} m \frac{2}{\sqrt{T-t}} \varphi\left(\frac{z}{\sqrt{T-t}}\right) d z=2 m \int_{0}^{\frac{m-x}{\sqrt{T-t}}} \varphi(z) d z=2 m\left(\Phi\left(\frac{m-x}{\sqrt{T-t}}\right)-\frac{1}{2}\right)$,

where $\Phi(y)=\int_{-\infty}^{y} \varphi(z) d z, y \in \mathbb{R}$, is the standard Gaussian cumulative distribution function. Concerning the second integral in (55), we have

$$
\begin{aligned}
\int_{m-x}^{\infty}(z+x) \frac{2}{\sqrt{T-t}} \varphi\left(\frac{z}{\sqrt{T-t}}\right) d z & =2 \sqrt{T-t} \int_{\frac{m-x}{\sqrt{T-t}}}^{\infty} z \varphi(z) d z+2 x \int_{\frac{m-x}{\sqrt{T-t}}}^{\infty} \varphi(z) d z \\
& =\sqrt{\frac{2(T-t)}{\pi}} e^{-\frac{(m-x)^{2}}{2(T-t)}}+2 x\left(1-\Phi\left(\frac{m-x}{\sqrt{T-t}}\right)\right) .
\end{aligned}
$$


Second case: $x>m$. We have

$$
\begin{aligned}
f(t, m, x) & =\int_{0}^{\infty}(z+x) \frac{2}{\sqrt{T-t}} \varphi\left(\frac{z}{\sqrt{T-t}}\right) d z \\
& =2 \sqrt{T-t} \int_{0}^{\infty} z \varphi(z) d z+2 x \int_{0}^{\infty} \varphi(z) d z=\sqrt{\frac{2(T-t)}{\pi}}+x .
\end{aligned}
$$

We also have the following regularity result regarding the function $f$.

Lemma 3 The function $f$ defined in (54) is continuous on $[0, T] \times \mathbb{R} \times \mathbb{R}$, moreover it is once (resp. twice) continuously differentiable in $(t, m)$ (resp. in $x)$ on $[0, T[\times \bar{Q}$, where $\bar{Q}$ is the closure of the set $Q:=\{(m, x) \in \mathbb{R} \times \mathbb{R}: m>x\}$. In addition, the following Itô formula holds:

$$
\begin{aligned}
f\left(t, S_{t}, B_{t}\right)= & f(0,0,0)+\int_{0}^{t}\left(\partial_{t} f\left(s, S_{s}, B_{s}\right)+\frac{1}{2} \partial_{x x}^{2} f\left(s, S_{s}, B_{s}\right)\right) d s \\
& +\int_{0}^{t} \partial_{m} f\left(s, S_{s}, B_{s}\right) d S_{s}+\int_{0}^{t} \partial_{x} f\left(s, S_{s}, B_{s}\right) d B_{s}, \quad 0 \leq t \leq T, \mathbb{P} \text {-a.s. }
\end{aligned}
$$

Proof The regularity properties of $f$ are deduced from its explicit form derived in Lemma 2, after straightforward calculations. Concerning Itô's formula (56), the proof can be done along the same lines as the standard Itô formula. We simply notice that, in the present case, only the restriction of $f$ to $\bar{Q}$ is smooth. However, the process $\left(\left(S_{t}, B_{t}\right)\right)_{t}$ is $\bar{Q}$-valued. It is well-known that if $\bar{Q}$ would be an open set, then Itô's formula would hold. In our case, $\bar{Q}$ is the closure of its interior $Q$. This latter property is enough for the validity of Itô's formula. In particular, the basic tools for the proof of Itô's formula are the following Taylor expansions for the function $f$ :

$$
\begin{aligned}
f\left(t^{\prime}, m, x\right)= & f(t, m, x)+\partial_{t} f(t, m, x)\left(t^{\prime}-t\right) \\
& +\int_{0}^{1} \partial_{t} f\left(t+\lambda\left(t^{\prime}-t\right), m, x\right)\left(t^{\prime}-t\right) d \lambda, \\
f\left(t, m^{\prime}, x\right)= & f(t, m, x)+\partial_{m} f(t, m, x)\left(m^{\prime}-m\right) \\
& +\int_{0}^{1} \partial_{m} f\left(t, m+\lambda\left(m^{\prime}-m\right), x\right)\left(m^{\prime}-m\right) d \lambda, \\
f\left(t, m, x^{\prime}\right)= & f(t, m, x)+\partial_{x} f(t, m, x)\left(x^{\prime}-x\right)+\frac{1}{2} \partial_{x x}^{2} f(t, m, x)\left(x^{\prime}-x\right)^{2} \\
& +\int_{0}^{1}(1-\lambda)\left(\partial_{x x}^{2} f\left(t, m, x+\lambda\left(x^{\prime}-x\right)\right)-\partial_{x x}^{2} f(t, m, x)\right)\left(x^{\prime}-x\right)^{2} d \lambda,
\end{aligned}
$$

for all $(t, m, x) \in[0, T] \times \bar{Q}$. To prove the above Taylor formulae, note that they hold on the open set $Q$, using the regularity of $f$. Then, we can extend them to the closure of $Q$, since $f$ and its derivatives are continuous on $\bar{Q}$. Consequently, Itô's formula can be proved in the usual way. 
Even though, as already observed, $\mathscr{U}$ does not belong to $C^{1,2}(([0, T[\times$ past $) \times$ present $) \cap C([0, T] \times C([-T, 0]))$, so that it can not be a classical solution to Eq. (43), the function $f$ is a solution to a certain Cauchy problem, as stated in the following proposition.

Proposition 10 The function $f$ defined in (54) solves the backward heat equation:

$$
\begin{cases}\partial_{t} f(t, m, x)+\frac{1}{2} \partial_{x x}^{2} f(t, m, x)=0, & \forall(t, m, x) \in[0, T[\times \bar{Q} \\ f(T, m, x)=m, & \forall(m, x) \in \bar{Q} .\end{cases}
$$

Proof We provide two distinct proofs.

Direct proof. Since we know the explicit expression of $f$, we can derive the form of $\partial_{t} f$ and $\partial_{x x}^{2} f$ by direct calculations:

$$
\partial_{t} f(t, m, x)=-\frac{1}{\sqrt{T-t}} \varphi\left(\frac{m-x}{\sqrt{T-t}}\right), \quad \partial_{x x}^{2} f(t, m, x)=\frac{2}{\sqrt{T-t}} \varphi\left(\frac{m-x}{\sqrt{T-t}}\right),
$$

for all $(t, m, x) \in[0, T[\times \bar{Q}$, from which the claim follows.

Probabilistic proof. By definition, the process $\left(f\left(t, S_{t}, B_{t}\right)\right)_{t \in[0, T]}$ is given by:

$$
f\left(t, S_{t}, B_{t}\right)=\mathbb{E}\left[S_{T} \mid \mathscr{F}_{t}\right]
$$

so that it is a uniformly integrable $\mathbb{F}$-martingale. Then, it follows from Itô's formula (56) that

$$
\int_{0}^{t}\left(\partial_{t} f\left(s, S_{s}, B_{s}\right)+\frac{1}{2} \partial_{x x}^{2} f\left(s, S_{s}, B_{s}\right)\right) d s+\int_{0}^{t} \partial_{m} f\left(s, S_{s}, B_{s}\right) d S_{s}=0,
$$

for all $0 \leq t \leq T, \mathbb{P}$-almost surely. As a consequence, the claim follows if we prove that

$$
\int_{0}^{t} \partial_{m} f\left(s, S_{s}, B_{s}\right) d S_{s}=0
$$

By direct calculation, we have

$$
\partial_{m} f(t, m, x)=2 \Phi\left(\frac{m-x}{\sqrt{T-t}}\right)-1, \quad \forall(t, m, x) \in[0, T[\times \bar{Q} .
$$

Therefore, (57) becomes

$$
\int_{0}^{t}\left(2 \Phi\left(\frac{S_{s}-B_{s}}{\sqrt{T-s}}\right)-1\right) d S_{s}=0
$$


Now we observe that the local time of $S_{s}-B_{s}$ is equal to $2 S_{s}$, see Exercise 2.14 in [35]. It follows that the measure $d S_{s}$ is carried by $\left\{s: S_{s}-B_{s}=0\right\}$. This in turn implies the validity of (58), since the integrand in (58) is zero on the set $\left\{s: S_{s}-B_{s}=0\right\}$.

\subsection{Strong-Viscosity Solutions}

Motivated by previous subsection and following [10], we now introduce a concept of weak (viscosity type) solution for the path-dependent Eq. (43), which we call strongviscosity solution to distinguish it from the classical notion of viscosity solution.

Definition 21 A function $\mathscr{U}:[0, T] \times C([-T, 0]) \rightarrow \mathbb{R}$ is called strong-viscosity solution to Eq. (43) if there exists a sequence $\left(\mathscr{U}_{n}, H_{n}, F_{n}\right)_{n}$ of Borel measurable functions $\mathscr{U}_{n}:[0, T] \times C([-T, 0]) \rightarrow \mathbb{R}, H_{n}: C([-T, 0]) \rightarrow \mathbb{R}, F_{n}:[0, T] \times$ $C([-T, 0]) \times \mathbb{R} \times \mathbb{R} \rightarrow \mathbb{R}$, satisfying the following.

(i) For all $t \in[0, T]$, the functions $\mathscr{U}_{n}(t, \cdot), H_{n}(\cdot), F_{n}(t, \cdot, \cdot, \cdot)$ are equicontinuous on compact sets and, for some positive constants $C$ and $m$,

$$
\begin{aligned}
\left|F_{n}(t, \eta, y, z)-F_{n}\left(t, \eta, y^{\prime}, z^{\prime}\right)\right| & \leq C\left(\left|y-y^{\prime}\right|+\left|z-z^{\prime}\right|\right), \\
\left|\mathscr{U}_{n}(t, \eta)\right|+\left|H_{n}(\eta)\right|+\left|F_{n}(t, \eta, 0,0)\right| & \leq C\left(1+\|\eta\|_{\infty}^{m}\right),
\end{aligned}
$$

for all $(t, \eta) \in[0, T] \times C([-T, 0]), y, y^{\prime} \in \mathbb{R}$, and $z, z^{\prime} \in \mathbb{R}$.

(ii) $\mathscr{U}_{n}$ is a strict solution to

$$
\begin{cases}\partial_{t} \mathscr{U}_{n}+D^{H} \mathscr{U}_{n}+\frac{1}{2} D^{V V} \mathscr{U}_{n}+F_{n}\left(t, \eta, \mathscr{U}_{n}, D^{V} \mathscr{U}_{n}\right)=0, & \forall(t, \eta) \in[0, T[\times C([-T, 0]), \\ \mathscr{U}_{n}(T, \eta)=H_{n}(\eta), & \forall \eta \in C([-T, 0]) .\end{cases}
$$

(iii) $\left(\mathscr{U}_{n}, H_{n}, F_{n}\right)$ converges pointwise to $(\mathscr{U}, H, F)$ as $n$ tends to infinity.

Remark 12 (i) Notice that in [8], Definition 3.4, instead of the equicontinuity on compact sets we supposed the local equicontinuity, i.e., the equicontinuity on bounded sets (see Definition 3.3 in [8]). This latter condition is stronger when $\mathscr{U}$ (as well as the other coefficients) is defined on a non-locally compact topological space, as for example $[0, T] \times C([-T, 0])$.

(ii) We observe that, for every $t \in[0, T]$, the equicontinuity on compact sets of $\left(\mathscr{U}_{n}(t, \cdot)\right)_{n}$ together with its pointwise convergence to $\mathscr{U}(t, \cdot)$ is equivalent to requiring the uniform convergence on compact sets of $\left(\mathscr{U}_{n}(t, \cdot)\right)_{n}$ to $\mathscr{U}(t, \cdot)$. The same remark applies to $\left(H_{n}(\cdot)\right)_{n}$ and $\left(F_{n}(t, \cdot, \cdot, \cdot)\right)_{n}, t \in[0, T]$.

The following uniqueness result for strong-viscosity solution holds. 
Theorem 6 Suppose that Assumption (A) holds. Let $\mathscr{U}:[0, T] \times C([-T, 0]) \rightarrow \mathbb{R}$ be a strong-viscosity solution to Eq. (43). Then, we have

$$
\mathscr{U}(t, \eta)=Y_{t}^{t, \eta}, \quad \forall(t, \eta) \in[0, T] \times C([-T, 0]),
$$

where $\left(Y_{s}^{t, \eta}, Z_{s}^{t, \eta}\right)_{s \in[t, T]} \in \mathbb{S}^{2}(t, T) \times \mathbb{H}^{2}(t, T)$, with $Y_{s}^{t, \eta}=\mathscr{U}\left(s, \mathbb{W}_{s}^{t, \eta}\right)$, solves the backward stochastic differential equation, $\mathbb{P}$-a.s.,

$$
Y_{s}^{t, \eta}=H\left(\mathbb{W}_{T}^{t, \eta}\right)+\int_{s}^{T} F\left(r, \mathbb{W}_{r}^{t, \eta}, Y_{r}^{t, \eta}, Z_{r}^{t, \eta}\right) d r-\int_{s}^{T} Z_{r}^{t, \eta} d W_{r}, \quad t \leq s \leq T .
$$

In particular, there exists at most one strong-viscosity solution to Eq. (43).

Proof Consider a sequence $\left(\mathscr{U}_{n}, H_{n}, F_{n}\right)_{n}$ satisfying conditions (i)-(iii) of Definition 21 . For every $n \in \mathbb{N}$ and any $(t, \eta) \in[0, T] \times C([-T, 0])$, we know from Theorem 4 that $\left(Y_{s}^{n, t, \eta}, Z_{s}^{n, t, \eta}\right)_{s \in[t, T]}=\left(\mathscr{U}_{n}\left(s, \mathbb{W}_{s}^{t, \eta}\right), D^{V} \mathscr{U}_{n}\left(s, \mathbb{W}_{s}^{t, \eta}\right)\right)_{s \in[t, T]} \in \mathbb{S}^{2}(t, T) \times$ $\mathbb{H}^{2}(t, T)$ is the solution to the backward stochastic differential equation, $\mathbb{P}$-a.s.,

$$
Y_{s}^{n, t, \eta}=H_{n}\left(\mathbb{W}_{T}^{t, \eta}\right)+\int_{s}^{T} F_{n}\left(r, \mathbb{W}_{r}^{t, \eta}, Y_{r}^{n, t, \eta}, Z_{r}^{n, t, \eta}\right) d r-\int_{s}^{T} Z_{r}^{n, t, \eta} d W_{r}, \quad t \leq s \leq T .
$$

Our aim is to pass to the limit in the above equation as $n \rightarrow \infty$, using Theorem C.1 in [10]. From the polynomial growth condition of $\left(\mathscr{U}_{n}\right)_{n}$ and estimate (46), we see that

$$
\sup _{n}\left\|Y^{n, t, \eta}\right\|_{\mathbb{S} p(t, T)}<\infty, \quad \text { for any } p \geq 1
$$

This implies, using standard estimates for backward stochastic differential equations (see, e.g., Proposition B.1 in [10]) and the polynomial growth condition of $\left(F_{n}\right)_{n}$, that

$$
\sup _{n}\left\|Z^{n, t, \eta}\right\|_{\mathbb{H}^{2}(t, T)}<\infty
$$

Let $Y_{s}^{t, \eta}=\mathscr{U}\left(s, \mathbb{W}_{s}^{t, \eta}\right)$, for any $s \in[t, T]$. Then, we see that all the requirements of Theorem C.1 in [10] follow by assumptions and estimate (46), so the claim follows.

We now prove an existence result for strong-viscosity solutions to the pathdependent heat equation, namely to Eq. (43) in the case $F \equiv 0$. To this end, we need the following stability result for strong-viscosity solutions.

Lemma 4 Let $\left(\mathscr{U}_{n, k}, H_{n, k}, F_{n, k}\right)_{n, k}$, $\left(\mathscr{U}_{n}, H_{n}, F_{n}\right)_{n},(\mathscr{U}, H, F)$ be Borel measurable functions such that the properties below hold.

(i) For all $t \in[0, T]$, the functions $\mathscr{U}_{n, k}(t, \cdot), H_{n, k}(\cdot)$, and $F_{n, k}(t, \cdot, \cdot, \cdot), n, k \in \mathbb{N}$, are equicontinuous on compact sets and, for some positive constants $C$ and $m$, 


$$
\begin{aligned}
\left|F_{n, k}(t, \eta, y, z)-F_{n, k}\left(t, \eta, y^{\prime}, z^{\prime}\right)\right| & \leq C\left(\left|y-y^{\prime}\right|+\left|z-z^{\prime}\right|\right), \\
\left|\mathscr{U}_{n, k}(t, \eta)\right|+\left|H_{n, k}(\eta)\right|+\left|F_{n, k}(t, \eta, 0,0)\right| & \leq C\left(1+\|\eta\|_{\infty}^{m}\right),
\end{aligned}
$$

for all $(t, \eta) \in[0, T] \times C([-T, 0]), y, y^{\prime} \in \mathbb{R}$, and $z, z^{\prime} \in \mathbb{R}$.

(ii) $\mathscr{U}_{n, k}$ is a strict solution to

$$
\begin{cases}\partial_{t} \mathscr{U}_{n, k}+D^{H} \mathscr{U}_{n, k}+\frac{1}{2} D^{V V} \mathscr{U}_{n, k} & \\ +F_{n, k}\left(t, \eta, \mathscr{U}_{n, k}, D^{V} \mathscr{U}_{n, k}\right)=0, & \forall(t, \eta) \in[0, T[\times C([-T, 0]), \\ \mathscr{U}_{n, k}(T, \eta)=H_{n, k}(\eta), & \forall \eta \in C([-T, 0]) .\end{cases}
$$

(iii) $\left(\mathscr{U}_{n, k}, H_{n, k}, F_{n, k}\right)$ converges pointwise to $\left(\mathscr{U}_{n}, H_{n}, F_{n}\right)$ as $k$ tends to infinity.

(iv) $\left(\mathscr{U}_{n}, H_{n}, F_{n}\right)$ converges pointwise to $(\mathscr{U}, H, F)$ as $n$ tends to infinity.

Then, there exists a subsequence $\left(\mathscr{U}_{n, k_{n}}, H_{n, k_{n}}, F_{n, k_{n}}\right)_{n}$ which converges pointwise to $(\mathscr{U}, H, F)$ as $n$ tends to infinity. In particular, $\mathscr{U}$ is a strong-viscosity solution to Eq. (43).

Proof See Lemma 3.4 in [8] or Lemma 3.1 in [10]. We remark that in [8] a slightly different definition of strong-viscosity solution was used, see Remark 12(i); however, proceeding along the same lines we can prove the present result.

Theorem 7 Suppose that Assumption (A) holds. Let $F \equiv 0$ and $H$ be continuous. Then, there exists a unique strong-viscosity solution $\mathscr{U}$ to the path-dependent heat Eq. (43), which is given by

$$
\mathscr{U}(t, \eta)=\mathbb{E}\left[H\left(\mathbb{W}_{T}^{t, \eta}\right)\right], \quad \forall(t, \eta) \in[0, T] \times C([-T, 0]) .
$$

Proof Let $\left(e_{i}\right)_{i \geq 0}$ be the orthonormal basis of $L^{2}([-T, 0])$ composed by the functions

$$
e_{0}=\frac{1}{\sqrt{T}}, \quad e_{2 i-1}(x)=\sqrt{\frac{2}{T}} \sin \left(\frac{2 \pi}{T}(x+T) i\right), \quad e_{2 i}(x)=\sqrt{\frac{2}{T}} \cos \left(\frac{2 \pi}{T}(x+T) i\right),
$$

for all $i \in \mathbb{N} \backslash\{0\}$. Let us define the linear operator $\Lambda: C([-T, 0]) \rightarrow C([-T, 0])$ by

$$
(\Lambda \eta)(x)=\frac{\eta(0)-\eta(-T)}{T} x, \quad x \in[-T, 0], \eta \in C([-T, 0]) .
$$

Notice that $(\eta-\Lambda \eta)(-T)=(\eta-\Lambda \eta)(0)$, therefore $\eta-\Lambda \eta$ can be extended to the entire real line in a periodic way with period $T$, so that we can expand it in Fourier series. In particular, for each $n \in \mathbb{N}$ and $\eta \in C([-T, 0])$, consider the Fourier partial sum

$$
s_{n}(\eta-\Lambda \eta)=\sum_{i=0}^{n}\left(\eta_{i}-(\Lambda \eta)_{i}\right) e_{i}, \quad \forall \eta \in C([-T, 0]),
$$


where (denoting $\tilde{e}_{i}(x)=\int_{-T}^{x} e_{i}(y) d y$, for any $x \in[-T, 0]$ ), by Proposition 4 ,

$$
\begin{aligned}
\eta_{i}=\int_{-T}^{0} \eta(x) e_{i}(x) d x & =\eta(0) \tilde{e}_{i}(0)-\int_{[-T, 0]} \tilde{e}_{i}(x) d^{-} \eta(x) \\
& =\int_{[-T, 0]}\left(\tilde{e}_{i}(0)-\tilde{e}_{i}(x)\right) d^{-} \eta(x),
\end{aligned}
$$

since $\eta(0)=\int_{[-T, 0]} d^{-} \eta(x)$. Moreover we have

$$
(\Lambda \eta)_{i}=\int_{-T}^{0}(\Lambda \eta)(x) e_{i}(x) d x=\frac{1}{T} \int_{-T}^{0} x e_{i}(x) d x\left(\int_{[-T, 0]} d^{-} \eta(x)-\eta(-T)\right) .
$$

Define

$$
\sigma_{n}=\frac{s_{0}+s_{1}+\cdots+s_{n}}{n+1}
$$

Then, by (59),

$$
\sigma_{n}(\eta-\Lambda \eta)=\sum_{i=0}^{n} \frac{n+1-i}{n+1}\left(\eta_{i}-(\Lambda \eta)_{i}\right) e_{i}, \quad \forall \eta \in C([-T, 0]) .
$$

We know from Fejér's theorem on Fourier series (see, e.g., Theorem 3.4, Chapter III, in [44]) that, for any $\eta \in C([-T, 0]), \sigma_{n}(\eta-\Lambda \eta) \rightarrow \eta-\Lambda \eta$ uniformly on $[-T, 0]$, as $n$ tends to infinity, and $\left\|\sigma_{n}(\eta-\Lambda \eta)\right\|_{\infty} \leq\|\eta-\Lambda \eta\|_{\infty}$. Let us define the linear operator $T_{n}: C([-T, 0]) \rightarrow C([-T, 0])$ by (denoting $e_{-1}(x)=x$, for any $x \in[-T, 0])$

$$
\begin{aligned}
T_{n} \eta=\sigma_{n}(\eta-\Lambda \eta)+\Lambda \eta & =\sum_{i=0}^{n} \frac{n+1-i}{n+1}\left(\eta_{i}-(\Lambda \eta)_{i}\right) e_{i}+\frac{\eta(0)-\eta(-T)}{T} e_{-1} \\
& =\sum_{i=0}^{n} \frac{n+1-i}{n+1} x_{i} e_{i}+x_{-1} e_{-1},
\end{aligned}
$$

where, using (60) and (61),

$$
\begin{aligned}
x_{-1} & =\int_{[-T, 0]} \frac{1}{T} d^{-} \eta(x)-\frac{1}{T} \eta(-T), \\
x_{i} & =\int_{[-T, 0]}\left(\tilde{e}_{i}(0)-\tilde{e}_{i}(x)-\frac{1}{T} \int_{-T}^{0} x e_{i}(x) d x\right) d^{-} \eta(x)+\frac{1}{T} \int_{-T}^{0} x e_{i}(x) d x \eta(-T),
\end{aligned}
$$


for $i=0, \ldots, n$. Then, for any $\eta \in C([-T, 0]), T_{n} \eta \rightarrow \eta$ uniformly on $[-T, 0]$, as $n$ tends to infinity. Furthermore, there exists a positive constant $M$ such that

$$
\left\|T_{n} \eta\right\|_{\infty} \leq M\|\eta\|_{\infty}, \quad \forall n \in \mathbb{N}, \forall \eta \in C([-T, 0]) .
$$

In particular, the family of linear operators $\left(T_{n}\right)_{n}$ is equicontinuous. Now, let us define $\bar{H}_{n}: C([-T, 0]) \rightarrow \mathbb{R}$ as follows

$$
\bar{H}_{n}(\eta)=H\left(T_{n} \eta\right), \quad \forall \eta \in C([-T, 0])
$$

We see from (63) that the family $\left(\bar{H}_{n}\right)_{n}$ is equicontinuous on compact sets. Moreover, from the polynomial growth condition of $H$ and (63) we have

$$
\left|\bar{H}_{n}(\eta)\right| \leq C\left(1+\left\|T_{n} \eta\right\|_{\infty}^{m}\right) \leq C\left(1+M^{m}\|\eta\|_{\infty}^{m}\right), \quad \forall n \in \mathbb{N}, \forall \eta \in C([-T, 0]) .
$$

Now, we observe that since $\left\{e_{-1}, e_{0}, e_{1}, \ldots, e_{n}\right\}$ are linearly independent, then we see from (62) that $T_{n} \eta$ is completely characterized by the coefficients of $e_{-1}, e_{0}, e_{1}, \ldots, e_{n}$. Therefore, the function $\bar{h}_{n}: \mathbb{R}^{n+2} \rightarrow \mathbb{R}$ given by

$$
\bar{h}_{n}\left(x_{-1}, \ldots, x_{n}\right)=\bar{H}_{n}(\eta)=H\left(\sum_{i=0}^{n} \frac{n+1-i}{n+1} x_{i} e_{i}+x_{-1} e_{-1}\right), \quad \forall\left(x_{-1}, \ldots, x_{n}\right) \in \mathbb{R}^{n+2},
$$

completely characterizes $\bar{H}_{n}$. Moreover, fix $\eta \in C([-T, 0])$ and consider the corresponding coefficients $x_{-1}, \ldots, x_{n}$ with respect to $\left\{e_{-1}, \ldots, e_{n}\right\}$ in the expression (62) of $T_{n} \eta$. Set

$$
\begin{aligned}
\varphi_{-1}(x) & =\frac{1}{T}, \quad \varphi_{i}(x)=\tilde{e}_{i}(0)-\tilde{e}_{i}(x-T)-\frac{1}{T} \int_{-T}^{0} x e_{i}(x) d x, \quad x \in[0, T], \\
a_{-1} & =-\frac{1}{T}, \quad a_{i}=\frac{1}{T} \int_{-T}^{0} x e_{i}(x) d x .
\end{aligned}
$$

Notice that $\varphi_{-1}, \ldots, \varphi_{n} \in C^{\infty}([0, T])$. Then, we have

$$
\bar{H}_{n}(\eta)=\bar{h}_{n}\left(\int_{[-T, 0]} \varphi_{-1}(x+T) d^{-} \eta(x)+a_{-1} \eta(-T), \ldots, \int_{[-T, 0]} \varphi_{n}(x+T) d^{-} \eta(x)+a_{n} \eta(-T)\right) .
$$

Let $\phi(x)=c \exp \left(1 /\left(x^{2}-T^{2}\right)\right) 1_{[0, T[}(x), x \geq 0$, with $c>0$ such that $\int_{0}^{\infty} \phi(x)$ $d x=1$. Define, for any $\varepsilon>0, \phi_{\varepsilon}(x)=\phi(x / \varepsilon) / \varepsilon, x \geq 0$. Notice that $\phi_{\varepsilon} \in$ $C^{\infty}\left(\left[0, \infty[)\right.\right.$ and (denoting $\tilde{\phi}_{\varepsilon}(x)=\int_{0}^{x} \phi_{\varepsilon}(y) d y$, for any $\left.x \geq 0\right)$,

$$
\begin{aligned}
\int_{-T}^{0} \eta(x) \phi_{\varepsilon}(x+T) d x & =\eta(0) \tilde{\phi}_{\varepsilon}(T)-\int_{[-T, 0]} \tilde{\phi}_{\varepsilon}(x+T) d^{-} \eta(x) \\
& =\int_{[-T, 0]}\left(\tilde{\phi}_{\varepsilon}(T)-\tilde{\phi}_{\varepsilon}(x+T)\right) d^{-} \eta(x) .
\end{aligned}
$$


Therefore

$\lim _{\varepsilon \rightarrow 0^{+}} \int_{[-T, 0]}\left(\tilde{\phi}_{\varepsilon}(T)-\tilde{\phi}_{\varepsilon}(x+T)\right) d^{-} \eta(x)=\lim _{\varepsilon \rightarrow 0^{+}} \int_{-T}^{0} \eta(x) \phi_{\varepsilon}(x+T) d x=\eta(-T)$.

For this reason, we introduce the function $H_{n}: C([-T, 0]) \rightarrow \mathbb{R}$ given by

$$
H_{n}(\eta)=\bar{h}_{n}\left(\ldots, \int_{[-T, 0]} \varphi_{i}(x+T) d^{-} \eta(x)+a_{i} \int_{[-T, 0]}\left(\tilde{\phi}_{n}(T)-\tilde{\phi}_{n}(x+T)\right) d^{-} \eta(x), \ldots\right) .
$$

Now, for any $n \in \mathbb{N}$, let $\left(h_{n, k}\right)_{k \in \mathbb{N}}$ be a locally equicontinuous sequence of $C^{2}\left(\mathbb{R}^{n+2} ; \mathbb{R}\right)$ functions, uniformly polynomially bounded, such that $h_{n, k}$ converges pointwise to $h_{n}$, as $k$ tends to infinity. Define $H_{n, k}: C([-T, 0]) \rightarrow \mathbb{R}$ as follows:

$H_{n, k}(\eta)=h_{n, k}\left(\ldots, \int_{[-T, 0]} \varphi_{i}(x+T) d^{-} \eta(x)+a_{i} \int_{[-T, 0]}\left(\tilde{\phi}_{n}(T)-\tilde{\phi}_{n}(x+T)\right) d^{-} \eta(x), \ldots\right)$.

Then, we know from Theorem 5 that the function $\mathscr{U}_{n, k}:[0, T] \times C([-T, 0]) \rightarrow \mathbb{R}$ given by

$$
\mathscr{U}_{n, k}(t, \eta)=\mathbb{E}\left[H_{n, k}\left(\mathbb{W}_{T}^{t, \eta}\right)\right], \quad \forall(t, \eta) \in[0, T] \times C([-T, 0])
$$

is a classical solution to the path-dependent heat Eq. (43). Moreover, the family $\left(\mathscr{U}_{n, k}\right)_{n, \varepsilon, k}$ is equicontinuous on compact sets and uniformly polynomially bounded. Then, using the stability result Lemma 4 , it follows that $\mathscr{U}$ is a strong-viscosity solution to the path-dependent heat Eq. (43).

Acknowledgments The present work was partially supported by the ANR Project MASTERIE 2010 BLAN 012101 . The second named author also benefited partially from the support of the "FMJH Program Gaspard Monge in optimization and operation research" (Project 2014-1607H).

Open Access This chapter is distributed under the terms of the Creative Commons Attribution Noncommercial License, which permits any noncommercial use, distribution, and reproduction in any medium, provided the original author(s) and source are credited.

\section{References}

1. Buckdahn, R., Ma, J., Zhang, J.: Pathwise Taylor expansions for random fields on multiple dimensional paths. Stoch. Process. Appl. 125(7), 2820-2855 (2015)

2. Cerrai, S.: Second Order PDE's in Finite and Infinite Dimension: A Probabilistic Approach, Lecture Notes in Mathematics, vol. 1762. Springer, Berlin (2001)

3. Cerutti, M.C., Escauriaza, L., Fabes, E.B.: Uniqueness in the Dirichlet problem for some elliptic operators with discontinuous coefficients. Ann. Mat. Pura Appl. 4(163), 161-180 (1993)

4. Chojnowska-Michalik, A.: Representation theorem for general stochastic delay equations. Bull. Acad. Polon. Sci. Sér. Sci. Math. Astronom. Phys. 26(7), 635-642 (1978)

5. Cont, R., Fournié, D.-A.: Change of variable formulas for non-anticipative functionals on path space. J. Funct. Anal. 259(4), 1043-1072 (2010) 
6. Cont, R., Fournié, D.-A.: A functional extension of the Itô formula. C. R. Math. Acad. Sci. Paris 348(1-2), 57-61 (2010)

7. Cont, R., Fournié, D.-A.: Functional Itô calculus and stochastic integral representation of martingales. Ann. Probab. 41(1), 109-133 (2013)

8. Cosso, A., Russo, F.: A regularization approach to functional Itô calculus and strong-viscosity solutions to path-dependent PDEs. Preprint HAL, hal-00933678 version 2 (2014) (Unpublished)

9. Cosso, A., Russo, F.: Functional Itô versus Banach space stochastic calculus and strict solutions of semilinear path-dependent equations. Preprint arXiv:1505.02926v1 (2015)

10. Cosso, A., Russo, F.: Strong-viscosity solutions: semilinear parabolic PDEs and path-dependent PDEs. Preprint arXiv:1505.02927v1 (2015)

11. Crandall, M.G., Kocan, M., Soravia, P., Święch, A.: On the equivalence of various weak notions of solutions of elliptic PDEs with measurable ingredients. In: Progress in Elliptic and Parabolic Partial Differential Equations (Capri, 1994). Pitman Research Notes in Mathematics Series, vol. 350, pp. 136-162. Longman, Harlow (1996)

12. Di Girolami, C., Fabbri, G., Russo, F.: The covariation for Banach space valued processes and applications. Metrika 77(1), 51-104 (2014)

13. Di Girolami, C., Russo, F.: Infinite dimensional stochastic calculus via regularization and applications. Preprint HAL-INRIA, inria-00473947 version 1 (2010) (Unpublished)

14. Di Girolami, C., Russo, F.: Clark-Ocone type formula for non-semimartingales with finite quadratic variation. C. R. Math. Acad. Sci. Paris 349(3-4), 209-214 (2011)

15. Di Girolami, C., Russo, F.: Generalized covariation and extended Fukushima decomposition for Banach space-valued processes. Applications to windows of Dirichlet processes. Infinite Dimens. Anal. Quantum Probab. Relat. Top. 15(2), 1250007, 50 (2012)

16. Di Girolami, C., Russo, F.: Generalized covariation for Banach space valued processes, Itô formula and applications. Osaka J. Math. 51(3), 729-783 (2014)

17. Dupire, B.: Functional Itô calculus. Portfolio Research Paper, Bloomberg (2009)

18. Ekren, I., Keller, C., Touzi, N., Zhang, J.: On viscosity solutions of path dependent PDEs. Ann. Probab. 42(1), 204-236 (2014)

19. Ekren, I., Touzi, N., Zhang, J.: Viscosity solutions of fully nonlinear parabolic path dependent PDEs: Part I. Ann. Probab, To appear in (2013)

20. Ekren, I., Touzi, N., Zhang, J.: Viscosity solutions of fully nonlinear parabolic path dependent PDEs: Part II. Ann. Probab, To appear in (2013)

21. Ekren, I., Touzi, N., Zhang, J.: Optimal stopping under nonlinear expectation. Stoch. Process. Appl. 124(10), 3277-3311 (2014)

22. Flandoli, F., Zanco, G.: An infinite-dimensional approach to path-dependent Kolmogorov's equations. Ann. Probab, To appear in (2013)

23. Föllmer, H.: Calcul d'Itô sans probabilités. In: Seminar on Probability, XV (Univ. Strasbourg, Strasbourg, 1979/1980) (French). Lecture Notes in Mathematics, vol. 850 pp. 143-150. Springer, Berlin (1981)

24. Gozzi, F., Russo, F.: Verification theorems for stochastic optimal control problems via a time dependent Fukushima-Dirichlet decomposition. Stoch. Process. Appl. 116(11), 1530-1562 (2006)

25. Gozzi, F., Russo, F.: Weak Dirichlet processes with a stochastic control perspective. Stoch. Process. Appl. 116(11), 1563-1583 (2006)

26. Gradinaru, M., Nourdin, I.: Approximation at first and second order of $m$-order integrals of the fractional Brownian motion and of certain semimartingales. Electron. J. Probab. 8(18), 26(electronic) (2003)

27. Jensen, R.: Uniformly elliptic PDEs with bounded, measurable coefficients. J. Fourier Anal. Appl. 2(3), 237-259 (1996)

28. Jensen, R., Kocan, M., Święch, A.: Good and viscosity solutions of fully nonlinear elliptic equations. Proc. Amer. Math. Soc. 130(2), 533-542(electronic) (2002)

29. Leão, D., Ohashi, A., Simas, A.B.: Weak functional Itô calculus and applications. Preprint arXiv: $1408.1423 \mathrm{v} 2$ (2014) 
30. Mohammed, S.E.A.: Stochastic Functional Differential Equations. Research Notes in Mathematics, vol. 99. Pitman (Advanced Publishing Program), Boston (1984)

31. Nualart, D.: The Malliavin Calculus and Related Topics. Probability and its Applications. Springer, New York (1995)

32. Pazy, A.: Semigroups of Linear Operators and Applications to Partial Differential Equations. Applied Mathematical Sciences, vol. 44. Springer, New York (1983)

33. Peng, S.: Note on viscosity solution of path-dependent PDE and $G$-martingales. Preprint arXiv:1106.1144 (2012)

34. Ren, Z., Touzi, N., Zhang, J.: Comparison of viscosity solutions of semi-linear path-dependent PDEs. Preprint arXiv:1410.7281 (2014)

35. Revuz, D., Yor, M.: Continuous Martingales and Brownian Motion. Grundlehren der Mathematischen Wissenschaften [Fundamental Principles of Mathematical Sciences], third edn. Springer, Berlin (1999)

36. Rudin, W.: Real and Complex Analysis, 3rd edn. McGraw-Hill Book Co., New York (1987)

37. Russo, F., Vallois, P.: Intégrales progressive, rétrograde et symétrique de processus non adaptés. C. R. Acad. Sci. Paris Sér. I Math. 312(8), 615-618 (1991)

38. Russo, F., Vallois, P.: Forward, backward and symmetric stochastic integration. Probab. Theory Relat. Fields 97(3), 403-421 (1993)

39. Russo, F., Vallois, P.: The generalized covariation process and Itô formula. Stoch. Process. Appl. 59(1), 81-104 (1995)

40. Russo, F., Vallois, P.: Elements of stochastic calculus via regularization. In: Séminaire de Probabilités XL. Lecture Notes in Mathematics, vol. 1899, pp. 147-185. Springer, Berlin (2007)

41. Ryan, R.A.: Introduction to Tensor Products of Banach Spaces. Springer Monographs in Mathematics. Springer, London (2002)

42. Tang, S., Zhang, F.: Path-dependent optimal stochastic control and associated Bellman equations: a viscosity solution approach. Preprint arXiv:1210.2078 (2013)

43. Yan, F., Mohammed, S.: A stochastic calculus for systems with memory. Stoch. Anal. Appl. 23(3), 613-657 (2005)

44. Zygmund, A.: Trigonometric Series, vol. I, II, 3rd edn. Cambridge Mathematical Library. Cambridge University Press, Cambridge (2002) (With a foreword by Robert A. Fefferman) 